\title{
THE WORLD OF NON-COVALENT INTERACTIONS: 2006
}

\section{Pavel HobZA ${ }^{a, *}$, Rudolf ZAHRADNíK ${ }^{b}$ and Klaus MÜLLER-DeTHLEFS ${ }^{c, *}$}

${ }^{a}$ Institute of Organic Chemistry and Biochemistry, Academy of Sciences of the Czech Republic and Center for Biomolecules and Complex Molecular Systems, 16610 Prague 6, Czech Republic; e-mail:pavel.hobza@uochb.cas.cz

${ }^{b}$ J. Heyrovsky Institute of Physical Chemistry, Academy of Sciences of the Czech Republic, 18223 Prague 8, Czech Republic; e-mail: zahrad@jh-inst.cas.cz

${ }^{c}$ The Photon Science Institute, The University of Manchester, Simon Building, Manchester M13 9PL, United Kingdom; e-mail: k.muller-dethlefs@manchester.ac.uk

Received January 9, 2006 Accepted February 24, 2006

1. Introduction . . . . . . . . . . . . . . . . . . . . . . . . . . 445

1.1. A Historical Remark . . . . . . . . . . . . . . . . . . . . . . . 445

1.2. A Remark on Nomenclature of Molecular Complexes . . . . . . . . . . . . 447

1.3. Purpose and Scope; Theory and Experiment . . . . . . . . . . . . . . . . 448

1.4. Covalent versus Non-Covalent Bonds . . . . . . . . . . . . . . . . . . . 448

1.5. Experimental Observables . . . . . . . . . . . . . . . . . . . . . . . . 449

1.6. Covalent and Non-Covalent Interactions in Nature . . . . . . . . . . . . . 452

1.6.1. Quantum Chemical Methods for Non-Covalent Complexes . . . . . . . 454

1.6.2. Aims of This Review . . . . . . . . . . . . . . . . . . . . . . . 455

2. Characteristics of Non-Covalent Complexes and Their Determination

by Experimental and Theoretical Techniques . . . . . . . . . . . . . . . . . . 455

2.1. Structure and Geometry . . . . . . . . . . . . . . . . . . . . . . . . . 455

2.2. Microwave and Terahertz Spectroscopy. . . . . . . . . . . . . . . . . . . 456

2.2.1. Ultrasoft Potentials: The Riddle of The Ammonia Dimer . . . . . . . . 456

2.2.2. From Water Clusters to a Potential for Liquid Water . . . . . . . . . . 458

2.2.3. Rotational Coherence Spectroscopy . . . . . . . . . . . . . . . . . 460

2.2.4. Quantum Chemical ab initio Methods . . . . . . . . . . . . . . . . 460

2.2.5. Gradient Optimization and Basis Set Superposition Error . . . . . . . . 462

2.3. Stabilization Energy . . . . . . . . . . . . . . . . . . . . . . . . . . . 463

2.3.1. Mass-Resolved ZEKE Spectroscopy . . . . . . . . . . . . . . . . . . 464

2.3.2. Computation of Stabilization Energy . . . . . . . . . . . . . . . . . 468

2.4. Is Density Functional Theory (DFT) Capable of Describing
Non-Covalent Interactions?. . . . . . . . . . . . . . . . . . . . 472

2.5. Vibrational Frequencies . . . . . . . . . . . . . . . . . . . . . . . . . 473

3. Potential Energy and Free Energy Surfaces . . . . . . . . . . . . . . . . . . 476

3.1. Empirical Potentials and an Attempt to Pass to More and More
Extensive Systems: Combination of Various Elements of Theory. . . . . . . . 476

3.2. Nucleic Acid Base Pairs. . . . . . . . . . . . . . . . . . . . . . . . . . 477

3.3. Microhydrated Nucleic Acid Bases and Base Pairs . . . . . . . . . . . . . . 481 
3.4. Carboxylic Acid Dimers . . . . . . . . . . . . . . . . . . . . . . . . . 483

3.5. Experimental Methods for Exploring Stationary Points on the PES:

Stimulated Emission Pumping . . . . . . . . . . . . . . . . . . . 487

4. Most Important Types of Non-Covalent Complexes . . . . . . . . . . . . . . . 489

4.1. Classification of Non-Covalent Complexes . . . . . . . . . . . . . . . . . 489

4.2. Small and Medium Size Non-Covalent Systems . . . . . . . . . . . . . . . 490

4.3. Hydrogen Bonding and Improper Hydrogen Bonding . . . . . . . . . . . . 493

4.4. Dihydrogen Bonding . . . . . . . . . . . . . . . . . . . . . . . . . . 501

4.5. Cooperative Hydrogen Bonding . . . . . . . . . . . . . . . . . . . . . . 502

4.6. Nucleic Acid Components, Nucleic Acids, Peptides and Proteins. . . . . . . . 502

5. Interpretation of Experimental Results . . . . . . . . . . . . . . . . . . . . . 503

5.1. Phenol...Argon and Benzene Dimer in vacuo . . . . . . . . . . . . . . . . 504

5.2. The Benzene Dimer: A Test Case for Our Understanding . . . . . . . . . . . 507

5.3. Nucleic Acid Base Pairs in vacuo . . . . . . . . . . . . . . . . . . . . . . . . 509

5.3.1. Ultrafast Hydrogen Atom Transfer in Clusters of Aromatic Molecules

Including Base Pairs . . . . . . . . . . . . . . . . . . . . . . . . 512

5.4. Biomolecules in the Gas Phase . . . . . . . . . . . . . . . . . . . . . . 514

5.4.1. Photochemical Selectivity in Nucleic Acid Bases . . . . . . . . . . . . 514

5.5. Helium Nanodroplets: Formic Acid Dimer and Glycine Dimer. . . . . . . . . 515

6. Molecular Recognition and Self-Organization. . . . . . . . . . . . . . . . . . 516

6.1. Recognition at the Molecular Level . . . . . . . . . . . . . . . . . . . . 516

6.2. Self-Assembly . . . . . . . . . . . . . . . . . . . . . . . 517

7. Outlook and Conclusions . . . . . . . . . . . . . . . . . . . . . . . . 517

8. References . . . . . . . . . . . . . . . . . . . . . . . . . 518

The review focusses on the fundamental importance of non-covalent interactions in nature by illustrating specific examples from chemistry, physics and the biosciences. Laser spectroscopic methods and both ab initio and molecular modelling procedures used for the study of non-covalent interactions in molecular clusters are briefly outlined. The role of structure and geometry, stabilization energy, potential and free energy surfaces for molecular clusters is extensively discussed in the light of the most advanced ab initio computational results for the $\operatorname{CCSD}(\mathrm{T})$ method, extrapolated to the CBS limit. The most important types of non-covalent complexes are classified and several small and medium size non-covalent systems, including $\mathrm{H}$-bonded and improper $\mathrm{H}$-bonded complexes, nucleic acid base pairs, and peptides and proteins are discussed with some detail. Finally, we evaluate the interpretation of experimental results in comparison with state of the art theoretical models: this is illustrated for phenol...Ar, the benzene dimer and nucleic acid base pairs. A review with 270 references.

Keywords: Non-covalent (van der Waals) interactions; Molecular clusters; ZEKE spectroscopy, Hole-burning; REMPI, Molecular modelling. 


\section{INTRODUCTION}

\subsection{A Historical Remark}

It was none other than van der Waals ${ }^{1}$, in the 1870's, who realized that the discrepancies observed between the state function of a real gas and the ideal gas law ${ }^{1}$ could be accounted for by the attracting forces between molecules or rare gas atoms. Van der Waals introduced an equation of state suitable for describing the behaviour of real (in contrast to ideal) gases. Although this law does not provide the most accurate functional description for a real gas, it nevertheless constituted a major breakthrough. Van der Waals made it explicitly obvious, for instance with respect to condensation of all real gases, that significant attracting forces exist between gas molecules (or atoms, in the case of monoatomic gases), which exhibit a tendency to form a new type of bond. The formation of these special van der Waals bonds, compared to chemical bonds, is not energetically demanding at all; these bonds are, under general laboratory conditions, easily formed and just as easily split.

Many years later, in 1930, London ${ }^{2}$ (and soon afterwards Hellmann ${ }^{3}$ ) made a fundamental step in describing and interpreting these bonds. Works of these and other pioneers are mentioned or outlined in the classic book on intermolecular interactions by Hirschfelder, Curtiss, and Bird ${ }^{4}$, and a survey of monographs and reviews up to the mid-eighties is presented in a book on intermolecular complexes ${ }^{5}$. Selected summarizing works since about 1985 are presented in ref. ${ }^{6}$

Before attempting to define non-covalently (van der Waals) bound species, it seems expedient to consider the broad spectrum of species, ranging from elementary particles to the giant systems, which play a role in molecular sciences. It is, indeed, a very long path from (super)strings, electrons and quarks, via protons, neutrons and atoms, to molecules, macromolecules and to supramolecular structures and, finally, via the large components of living matter to real living matter itself. The subject of this review concerns a significant portion of this set and it would be unfortunate to belittle any of these particles or systems by not giving them due attention. Let us consider the case of neutrons except for their role in isotopes: neutrons were considered somewhat boring by many chemists and biologists. But to be able to describe theoretically something as fascinating and important as the discriminative ability of weak forces interacting with optical antipodes we need neutrons, because without including 
them in the respective Hamiltonian, we cannot succeed in giving a reasonable description of such systems.

In Fig. 1, the above-mentioned particles and species are associated with contemporary tools of theoretical physics, which may serve for their theoretical description. Our topic starts with the Schrödinger equation; increased interest in solving the time-dependent Schrödinger equation in connection with some topics, e.g., photochemistry and photophysics and/or ultra-fast processes, is anticipated. In the majority of cases the Schrödinger equation has been solved within the framework of what is called pioneer quantum mechanics, which, while not the best, is a fundamental theory? ${ }^{7}$. From the philosophical point of view, no doubt, this theory is suitable in connection with molecules and related systems but, as a fundamental theory it is not acceptable for the description of higher hierarchical levels; as Primas says, such a theory is not appropriate for describing beings such as dogs ${ }^{7}$. Figure 1 briefly suggests a way to overcome severe, inherent weaknesses of pioneering works in quantum mechanics, which might be useful in the context of the description of intermolecular and non-covalent interactions.

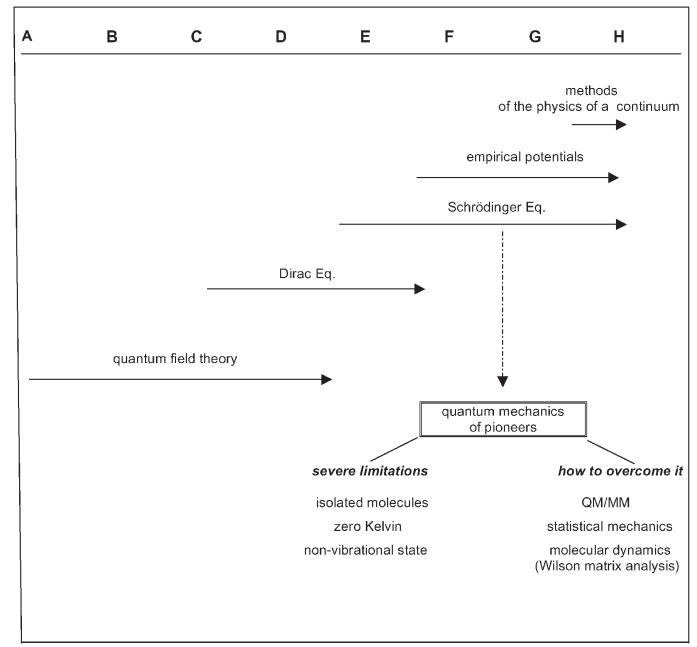

FIG. 1

Elementary particles (strings, A; quarks, B; electron, C; atomic nuclei, D; atoms, E; molecules, F; supramolecules, $\mathrm{G}$ and solid phase, $\mathrm{H}$ ) together with appropriate tools for theoretical description. The Schrödinger equation assumes a central role; its limitation in the framework of quantum mechanics of pioneers and possible ways of overcoming them are presented. QM/MM means quantum mechanical description of reacting system under study and molecular mechanical description of its neighborhood. 


\subsection{A Remark on Nomenclature of Molecular Complexes}

Why are molecular complexes, or molecular clusters, as they are most often called, of such interest? The main feature of molecular clusters is that they can be prepared experimentally in supersonic jet expansions and molecular beams as isolated systems exhibiting intermolecular bonds that originate from non-covalent interactions. From the theoretical point of view molecular clusters can also be studied using standard ab initio quantum chemical methods, treating the cluster as 'supermolecule' composed of several moieties held together by non-covalent bonds.

An issue in the literature that sometimes is unclear relates to the definition of the non-covalently bound complexes. A significant feature of such complexes is that the subsystems, of which they are constituted, are bound due to electric multipole-electric multipole interactions ${ }^{5}$. We consider permanent, inductive, and time-dependent multipoles. While it is possible to ascribe the stability of a complex to a bond, which is non-covalent in nature, it is not al ways easy to localize such a bond in space. When possible it is highly desirable to use another symbol for this bond than that which represents a covalent bond, i.e. a short full line -; hence, three dots ... may serve as a representation of a non-covalent bond. The hydrogen molecule and the helium (van der Waals) molecule are adequate representatives: $\mathrm{H}-\mathrm{H}$ and $\mathrm{He} . . . \mathrm{He}$, or alternatively $\mathrm{H}_{2}$ and $(\mathrm{He})_{2}$.

The second type of bond illustrated above still does not have a definite name. No doubt, it is possible to call it a non-covalent bond. Although it is really non-covalent, this non-specific name is not really an adequate description of its true nature. Another label, which is sometimes used, is derived from the term weak interactions and therefore the name 'weak bonds' is used. This is an unfortunate name, because it is derived from a designation, which has been used for a long time in physics for something completely different. We have favoured for years the designation 'van der Waals' (vdW in abbreviated form), e.g., vdW interactions, forces, bonds. It is unfortunately true that this designation has been corrupted - sometimes by poorly defined use - for a component of the empirical force field. In the case of empirical potentials the vdW term means a sum of London dispersion and exchange-repulsion terms. In our previous review ${ }^{6 a}$ we decided to use the term 'non-covalent' to classify interactions that are not covalent. We are aware that this definition is again not straightforward and unambiguous since, for example, metallic interactions are also covered but we believe that the term non-covalent properly describes the origin and nature of these interactions. 


\subsection{Purpose and Scope; Theory and Experiment}

A purpose of this review is to present why non-covalent interactions are of fundamental importance for chemistry and why their understanding is a conditio sine qua non for molecular biodisciplines. Moreover, an attempt will be made to describe correct procedures for treating these interactions theoretically. Those who deal with this subject daily do not need such a recommendation. However, it is nowadays increasingly common for chemists to do the necessary calculations themselves. In contrast to the realm where only chemical (i.e. covalent) bonds play a role, in the area of noncovalently bound complexes it is, in general, not trivial to assert how to proceed and which method and what level of theory guarantees obtaining reliable results. The situation is even more involved because problems of practical value in chemistry and still more in biology are - with respect to computer size - rather extensive. A choice of an appropriate method is especially challenging in these instances. Here, we will describe the main computational procedures to obtain static characteristics of non-covalent species; those characteristics are essential for the understanding of their dynamics.

\subsection{Covalent versus Non-Covalent Bonds}

The concept of covalent bonding belongs to the most successful concepts in modern science and is, at a certain level, a more or less closed chapter. After about eighty years of intense study, the processes of formation and breaking of covalent bonds are well understood and reliable descriptions of these processes can be performed at various theoretical levels. Calculated characteristic molecular properties obtained agree well with the relevant experiments and there are no fundamental disagreements between the state-of-the art theory and experiment. In contrast, the understanding of the nature of non-covalent interactions is far less clear and the respective calculations yield results that are frequently in conflict with experimental data. Basic principles of non-covalent interactions, for instance the hydrogenbond (H-bond), by Pauling ${ }^{8}$, were formulated in the 1930's. However, despite enormous progress made in theory as well as in experimental techniques in the last decades, we are still far from obtaining unambiguous and quantitatively satisfactory information about non-covalent complexes. Experiments do not allways yield complete information about a complex, though progress is being made by combining various techniques reducing ambiguity. Theory, on the other hand, is principally capable of providing 
full information about a non-covalent complex. For example, we can generate basic information such as structure and stabilization energy and from the knowledge of the wave function we can obtain any other desired property.

Comparison of theoretical and experimental results is of vital importance for theory as well as for experiments because it allows for the testing of the ability and accuracy of newly developed procedures and techniques. The combination of experiment and theory also gives a deeper insight into the problem studied and so leads to a deeper understanding.

\subsection{Experimental Observables}

The first question is which properties of non-covalent complexes are observable unambiguously? The surprising answer is not many of them! The structure is not directly observable and can only be determined by measuring the rotational constants thus providing the three principal moments of inertia. Rotational constants, however, do not provide an unambiguous answer concerning structure and geometry (see Section 2.1.). A similar situation exists for the determination of stabilization energies: of the various experimental techniques available only zero-electron kinetic energy (ZEKE) spectroscopy ${ }^{9,10}$ provides directly measurable information on stabilization energies ${ }^{11}$ (see Section 2.3.). In addition, directly observable characteristics of a non-covalent complex are vibrational frequencies, not all of which may be observable due to Franck-Condon factors or symmetry selection rules.

In this paper we venture into a comparison of theory and experiment. Before we go into any further detail of the theoretical and computational procedures, we would like to briefly summarize what can be learned from experiments with regard to understanding the intermolecular interactions that lead to non-covalent binding.

The first information that can be obtained are vibrational frequencies. There are essentially two methods for vibrational spectroscopy, based on infrared absorption or the Raman effect. Though some efforts have been put towards the development of stimulated Raman population transfer as a method of vibrationally resolved spectroscopy ${ }^{12}$, this method has not been utilized by many other groups. Most studies of vibrational spectroscopy for the determination of vibrational energy levels rely on ionization detection $^{13}$. For molecular clusters mass selection is essential and the detection methods are often based on most sensitive ionization techniques. The determination of vibrational frequencies is often straightforward and provides 
information on the strength of non-covalent interactions in a complex ${ }^{14}$. In general, direct absorption methods are unsuitable for molecular beam studies (except for recently developed IR cavity ring-down spectroscopy in slit nozzles $\left.{ }^{15}\right)$.

Second, very important information comes from the experimental determination of rotational constants. These are obtained from high-resolution spectroscopy with rotational level resolution: microwave spectroscopy as the method of highest resolving power ${ }^{16-18}$, vibration-rotation-tunnelling spectroscopy ${ }^{19}$, rotational electronic spectroscopy ${ }^{20-23}$ and deconvolution of only partially resolved rotational structure ${ }^{24-26}$.

Most suitable are methods based on mass spectrometry, particularly time-of-flight methods, which allow a mass signature to be obtained and thus allow for identification of certain molecular clusters and, in addition, provide single molecule detection efficiency. For vibrational spectroscopy a most successful method, the detection of infrared absorption by population depletion, is infrared-UV hole burning with ionization detection, first utilized by Brutschy and co-workers ${ }^{27}$.

Extensive IR-UV hole-burning studies were carried out by the groups of Zwier $^{28}$, Mikami ${ }^{13 b, 29}$ and Kleinermanns ${ }^{30}$, which most recently have even been applied to molecules of biological interest in the gas phase. The prin-

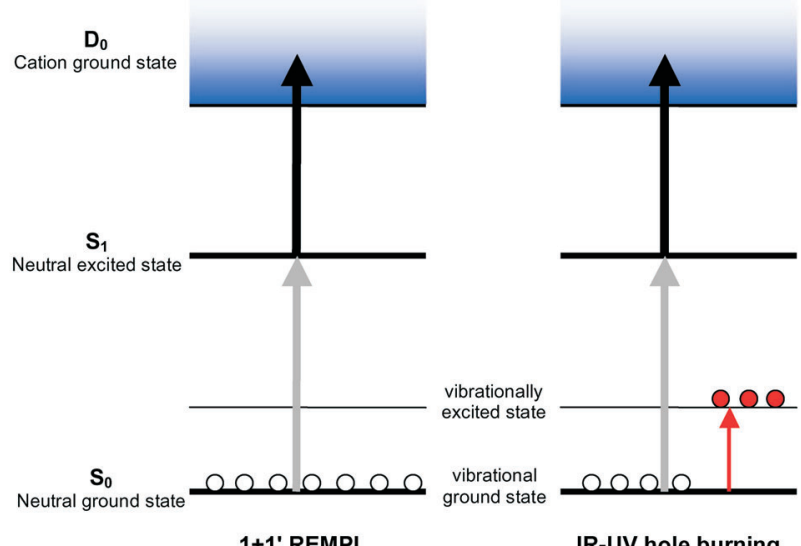

FIG. 2

Principle of IR-UV hole burning. An infrared laser is used to shift the population of the molecule of interest from its vibrational ground state into a vibrationally excited state. The molecule is also ionized from its vibrational ground state by a UV laser in a two-photon ionization process. Due to the depletion of the ground state by infrared absorption, a depletion in the ion signal is observed in the experiment, i.e. infrared absorption is characterized by an ion dip 
ciple of IR-UV hole burning is shown in Fig. 2. An infrared laser is used to shift the population of the molecule of interest from its vibrational ground state into a vibrationally excited state. The molecule is also ionized from its vibrational ground state by the UV laser in a two-photon ionization process. Due to the depletion of the vibrational ground state by the infrared absorption, a depletion in the ion signal is observed in the experiment, i.e. an infrared absorption is characterized by an ion dip. This type experiment has been very successful, and with the advent of new optical parametric IR laser sources the $\mathrm{OH}$ and $\mathrm{NH}^{31}$ vibration region of various species has been extensively probed.

Most recently the availability of infrared lasers in the region above 3.5 micrometers, based on difference frequency mixing and parametric amplification in non-linear silver selenide crystals has extended the IR tuning range substantially towards probing lower vibrational frequencies, particularly in the $\mathrm{CO}$ region ${ }^{32}$. For the infrared region above $6 \mu \mathrm{m}$ the big challenge comes from producing infrared laser sources that are suitable for laboratory use and which emit pulses of high enough energies (a few $\mathrm{mJ}$ 's) to be capable of inducing a population depletion that can easily be detected by photoionization. This challenge has very recently been resolved by Gerhards who has been able to extend the IR laser tuning range from 2 to $16 \mu \mathrm{m}$, using three different crystals, while producing pulse energies in the $\mathrm{mJ}$ range $32 \mathrm{2c} 33$. The IR free electron laser ${ }^{34}$, in particular FELIX ${ }^{35}$ at the FOM Institute Rujnhuisen provides a very high-powered (albeit not of very high resolution) laser source with IR frequencies down to about $100 \mathrm{~cm}^{-1}$. Several groups have now investigated molecules of biological interest in the gas phase using the infrared depletion technique ${ }^{24,36}$; for example, de Vries et al. have investigated the guanine...cytosine cluster using IR depletion, generating a wealth of new vibrational information compared with previously recorded electronic excitation spectra 37,38 .

It should be noted that the vibration frequency region below $1000 \mathrm{~cm}^{-1}$ is of particular interest. It is obvious from observed spectra that an assignment can only be made with the help of quantum chemical ab initio calculations. In this spectral region, where the vibrations are much softer than in the high-frequency region, it is absolutely imperative to perform anharmonic frequency calculations in order to interpret spectra. With some of these newly developed tools, for instance, Gerber's anharmonic selfconsistent field method ${ }^{39}$, it is now possible to fully assign the currently available spectra of complex systems such as guanine...cytosine ${ }^{40}$.

Time-resolved picosecond pump-probe ${ }^{41-43}$ and hole burning experiments are also a very useful tool for the study of the dynamics in molecular 
clusters. This spectroscopic method has been carried out for investigating the intracluster vibrational energy redistribution (IVR) and subsequent dissociation of molecular clusters. It provides the dynamic information of energy transfer from one moiety to a non-covalent bond, often in a two-stage process, and reveals that the intramolecular vibrational energy redistribution takes place within the chromophore site, creating a hot moiety (or compound), then the energy flows from the hot moiety to the intermolecular vibrational modes of the cluster. Finally, the molecular cluster dissociates. Very recently, we have used a picosecond pump-probe method to investigate the dynamics of competition between $\mathrm{H}$-bonding and $\pi$-stacking ${ }^{44}$ in the phenol...A $\mathrm{Ar}_{2}$ cation radical ${ }^{45}$. This can also be viewed as competition between $\mathrm{H}$-bonding (hydrophilic) and $\pi$-stacking (hydrophobic) binding sites, which is an example of a chemical recognition process at the molecular level. Reference ${ }^{45}$ describes the first direct observation of a hydrophobic $\rightarrow$ hydrophilic site-switching induced by resonant ionization in the phenol...Ar $\mathrm{Ar}_{2}$ trimer cation radical. When the cation radical is prepared by photoionization, it is produced in the $\pi$-bound geometry of the neutral precursor, with Ar binding to the hydrophobic ring site. On the time scale of a few picoseconds, one of the Ar atoms switches from the hydrophobic ring site to the hydrophilic $\mathrm{OH}$ site thus creating a hydrogen bond. The dynamics of this isomerization process is monitored in real time by a change in the $\mathrm{OH}$ stretching vibrational wavenumber using timeresolved picosecond UV-IR pump-probe ionization depletion spectroscopy 45 .

\subsection{Covalent and Non-Covalent Interactions in Nature}

Broadly, chemistry means covalent bonding. The covalent description is fully adequate when a molecule is considered in free space, i.e. isolated from any surroundings. Experimental conditions in molecular beams made from a supersonic jet expansion are close to these conditions. However, once the molecule is surrounded by other molecules, such as in solution or in the bulk, these surroundings affect the covalent bonding and the electronic system of the molecule is perturbed. This perturbation depends on the strength and extent of non-covalent interactions with the most pronounced changes occurring in ionic and $\mathrm{H}$-bonded systems. For certain cases the $\mathrm{H}$-bonding interactions, for instance between an anion and a neutral system, and covalent interactions can be of comparable strength. Mostly, however, non-covalent interactions are considerably weaker (by one to three orders of magnitude) than covalent bonds. Despite, or proba- 
bly because of this, non-covalent bonds play a subtle but decisive key role in nature.

First, the very existence of a liquid phase, and also all related effects like solvation phenomena, can be attributed to non-covalent interactions. The existence of a condensed phase probably represents the most important example of non-covalent interactions and it must be stated that theory still has serious problems in describing adequately the role of solvents in general and, more specifically, the role of liquid water. Whereas covalent systems can now be investigated with chemical accuracy $(\sim 1 \mathrm{kcal} / \mathrm{mol})$, the error in evaluation of hydration energies is much larger and, even worse, results from various theoretical methods differ significantly. Development of new procedures allowing determination of hydration energy is an important task for today's theoretical chemists.

Second, non-covalent interactions are responsible for the structure of biomacromolecules such as DNA, RNA, and proteins. It must be recalled that the double-helical structure of DNA, which is of key importance for transfer of genetic information, is mainly due to non-covalent interactions in which the interactions of nucleic acid bases play a dominant role. These bases are polar, aromatic heterocycles which interact either via planar $\mathrm{H}$-bonds or vertical $\pi-\pi$ interactions, resulting in two structural motifs, planar $\mathrm{H}$-bonding and $\pi$-stacking; both are important not only in determining the architecture of nucleic acids but in a much more general sense. The basic question is: what is the relative strength of these interactions? It was long believed that specific $\mathrm{H}$-bonding originating from electrostatic effects was the dominant term, while the non-specific stacking originating due to London dispersion effects was considered to be energetically much less significant. Only the most recent calculations (see Section 2.3.2.) have revealed that stacking can be associated with surprisingly large stabilization energies comparable with those of strong $\mathrm{H}$-bonding ${ }^{46}$.

Third, non-coval ent interactions play a key role in molecular recognition processes. This molecular recognition is most important in life processes, where it ensures an extremely high fidelity in the formation of, for instance, the required protein complexes.

Finally, the effect of purely dispersion-driven non-covalent interactions can be seen in the fantastic ability of the geckos to climb rapidly up smooth vertical surfaces, even flat glass. Very recently, it was found that even a macroscopic, quite large animal such as a gecko can fully support its substantial body weight from the non-covalent interactions between the few hundred thousands of keratinous hairs, or setae, on their feet and the surface. The adhesive force values observed support the hypothesis that indi- 
vidual setae operate via non-coval ent interactions, or as the authors put it, van der Waals forces ${ }^{47}$.

\subsubsection{Quantum Chemical Methods for Non-Covalent Complexes}

Non-covalent interactions can be studied in a manner similar to covalent interactions, that is, by standard methods of quantum chemistry based either on perturbation or variation theory. While the former approach separates the overall stabilization energy into various physically well-defined contributions such as electrostatic, induction, dispersion and exchangerepulsion energies, the latter separates the stabilization energy only into Hartree-Fock (HF) and post-HF (correlation) contributions. Furthermore, neither the Hartree-Fock nor correlation energy is observable, nor are the separate energy contributions (however physically meaningful they may be) defined in the perturbation theory expansion. The advantage of a physically meaningful perturbation expansion is compensated (or overcompensated) for by the difficulties of perturbation calculations. An important advantage of this approach (over variational) remains, namely the perturbation treatment is free of the most serious problem of the variation calculation - the basis set inconsistency. This inconsistency is associated with the fact that in the variation calculation the supersystem is described in the sum over basis sets of all subsystems leading to a more complete description compared with the isolated subsystems (being described only by their own individual basis sets) and thus to an artificially lowered energy (the so-called basis set superposition error, BSSE) ${ }^{48}$. This effect, which is purely mathematical in origin, has no physical meaning and can be eliminated either by describing the subsystems in the basis set of the whole supersystem, or by using infinite AO basis sets. The second approach, which seemed quite unrealistic until recently, is now available through an extrapolation procedure to the complete basis set limit (see Section 2.2.5.), for which, by definition, the BSSE disappears. Any variational treatment, which supposedly eliminates this basis set inconsistency, should be tested on the basis of obtaining the stabilization energy by perturbation theory, which is, by definition, free of this problem.

So what is the physical meaning of energy components of the stabilization energy? We believe that these should not be overestimated. In the majority of cases we are interested only in the total stabilization energy, which is without doubt more accurately and easily provided by the variation calculation. Problems associated with basis set inconsistency have now become less serious, which is due to: (i) hardware and software development 
allowing the use of much larger AO basis sets which, by definition, is consistent with lower BSSE; (ii) development of procedures which allow gradient optimization that takes the basis set inconsistency effect into account a priori, in each gradient cycle; and (iii) development of extrapolation techniques allowing for the estimation of the complete basis set (CBS) limit which should, by definition, be free of BSSE.

\subsubsection{Aims of This Review}

This review aims to understand the main aspects of non-covalent chemistry and specifically compares experimental and theoretical data available for non-covalent complexes and subsequent problems associated with this comparison. We stress that the review reflects the personal views of all three authors: we are considering both experimental (K. M.-D.) and theoretical (P. H. and R. Z.) aspects of non-covalent chemistry and we believe our views might be of help to researchers engaged in both theoretical and experimental areas of this subject area.

\section{CHARACTERISTICS OF NON-COVALENT COMPLEXES AND THEIR DETERMINATION BY EXPERIMENTAL AND THEORETICAL TECHNIQUES}

\subsection{Structure and Geometry}

The primary property of any non-covalent complex is not just its 'equilibrium structure', but its potential energy surface (PES) with its stationary points. Determination of structure and geometry of a complex cannot be separated from discussing its PES. To illustrate this point consider the intramolecular hydrogen atom dynamics of acetylacetone, for which a hydrogen shifting keto-enol tautomerization and an interconversion of the enol in two indistinguishable enolone structures was proposed ${ }^{49}$. Recent ultrafast electron diffraction experiments on acetylacetone by Zewail and co-workers ${ }^{50}$ have shown that of the two tautomeric forms in dynamic equilibrium the $\mathrm{C}_{\mathrm{s}}$-enolic structure is the dominant one. This was attributed to some $\pi$-delocalization, leading to a type of intramolecular interaction called 'resonance assisted hydrogen bond'. The net result is that the hydrogen prefers to be close to one of the oxygens (the enolone $\mathrm{C}_{\mathrm{s}}$ structure) and not inbetween the two oxygens $\left(C_{2 v}\right)$. They also observed the interconversion of the enol between the two equivalent enolone minima on the PES. The observation of a $\mathrm{C}_{\mathrm{s}}$ structure seems to contradict results from microwave spectra of acetylacetone that are compatible with a $C_{2 v}$ 
structure ${ }^{51}$. However, this 'contradiction' is only a rhetorical one; it can be easily understood that the microwave experiment will show an averaged $\mathrm{C}_{2 \mathrm{v}}$ structure provided the interconversion barrier between the two enolones is sufficiently low. The microwave experiment averages the tunnelling motion of the hydrogen atom between the two enolones in a similar way as donor-acceptor tunnelling in the ammonia dimer (see Section 2.2.1.). Similar results and conclusions were obtained for comparable systems exhibiting intramolecular hydrogen bonds such as malondialdehyde 52,53 .

For the non-specialist reader who might think experimental spectroscopic data can always be interpreted unambiguously it must be stated that structural information resulting from rotational constants is not unambiguous since one set of rotational constants can be assigned to various structures ${ }^{54}$. Hence, some additional information, sometimes referred to as chemical intuition, or another technique (mostly theoretical calculations) should be combined with the evidence from rotational constants to generate reliable structural information. Rotational constants for larger complexes can be obtained by using various experimental techniques: MW, $\mathrm{THZ}$, IR. One such is the determination of rotational constants in electronic excitation spectra, for instance in REM PI spectra $20-22,24,25,55$ and also, for the cation complex, from ZEKE spectra ${ }^{56}$, or from IR predissociation direct absorption in the cation monitored by predissociation ${ }^{57}$.

We will focus mainly on larger non-covalent complexes having more than 24 atoms (the benzene dimer, with 24 atoms, is considered here as an arbitrary boundary between small and medium, and extended complexes). Direct experimental determination of structure and geometry of extended non-covalent complexes is impractical: they can presently be obtained only indirectly from rotational constants.

\subsection{Microwave and Terahertz Spectroscopy}

\subsubsection{Ultrasoft Potentials: The Riddle of The Ammonia Dimer}

One of the most powerful methods in molecular sciences, one could even call it the classical method of molecular spectroscopy, is microwave spectroscopy. Microwave spectroscopy features superior, very high spectral resolution and allows to obtain - besides rotational constants - hyperfine splittings and other interactions in molecules and molecular clusters with unprecedented precision. This is a mature technique which is applied in a routine way in many laboratories to study a large variety of molecular prop- 
erties, including non-covalent interactions in molecular clusters and biomolecules in the gas phase ${ }^{16-18}$. What has to be said, however, is that structural determination is not as straightforward as one might believe, particularly for molecular clusters, in spite of the precision and accuracy of the spectral lines and the corresponding rotational constants and moments of inertia obtained. For rigid molecular systems there is a very clear recipe for molecular spectroscopy to reduce the rotational constants to moments of inertia, thus constructing a molecular structure. However, for non-rigid systems that exhibit large amplitude motion - this is a particular feature of many molecular clusters held together by non-covalent interactions - and that are furthermore particularly susceptible to centrifugal distortion effects, the connection between spectroscopic constants determined from experiment and structural determination is not so unambiguous.

A prime example of this comes from the problem of the structure of ammonia dimer, $\left(\mathrm{NH}_{3}\right)_{2}$, initiated by the seminal microwave spectroscopic experiments conducted by Klemperer and co-workers in the early 1980 's $\mathrm{s}^{58}$. Though this work has already been reviewed by us before ${ }^{6}$ we are mentioning it here once more because this molecular cluster shows two important features: large amplitude vibration and tunnelling motion. The original spectra were interpreted in terms of donor-acceptor tunnelling and rotational tunnelling of one $\mathrm{NH}_{3}$ moiety (but not inversion tunnelling, which was assumed to be quenched by the dimer formation), according to the molecular symmetry group $\mathrm{G}_{16}$ (refs ${ }^{59,60}$ ). Much to their surprise - and the surprise of the scientific community - their result implied a dimer structure that seemed not to be hydrogen-bonded at all. Their structure, shown in Fig. 3, seemed to resemble a cyclic structure with the nitrogen atoms more
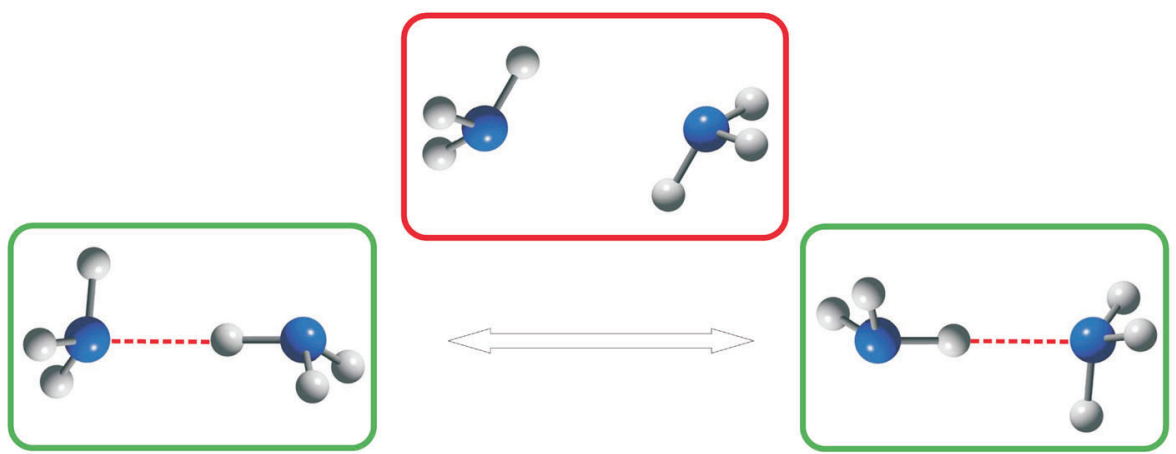

FIG. 3

Ammonia dimer structure as it appears after vibrational averaging 
or less facing each other in a distorted structure that did not at all seem to be hydrogen-bonded. However, it was quite apparent that the result from the microwave experiment would only be fully conclusive if a full interpretation of the rotational tunnelling structure could be determined from the starting point of a high-level potential energy surface calculated with a reliable ab initio method. This procedure took more than a decade and resulted in a most decisive contribution from van der Avoird and co-workers, who finally computed the potential energy surface to a sufficient precision to allow numerical determination of the full rotational tunnelling spectrum of the $\mathrm{NH}_{3}$ dimer, and also the perdeuterated species, within the molecular symmetry group $\mathrm{G}_{144}$ (ref. ${ }^{61}$ ). The result shows convincingly that, for an extremely flat potential with respect to the dimer bending motion, a normal microwave spectroscopy experiment would result in the determination of moments of inertia that represents an averaged vibrational-rotational structure. This shows clearly that the interpretation drawn from the first microwave spectra of the Klemperer group had, of course, been both correct but not fully conclusive at the same time. Correct in so far that - of course - the moments of inertia determined were correctly deduced from the experimental data, but incomplete in the context that only with a high-quality potential energy surface and a complete quantum calculation of energy states on that surface can the spectra be fully interpreted. These, naturally, were not available at the time when the first microwave measurements were carried out.

\subsubsection{From Water Clusters to a Potential for Liquid Water}

A story similar to that of the ammonia dimer, albeit of higher complexity, can be developed for water clusters. Water clusters are of substantial interest to understand not only the pairwise additive interactions, such as in the water dimer, but also the three-body (and many-body) interactions, such as those present in larger clusters like the trimer, tetramer, etc. The pioneering work of Saykally and co-workers ${ }^{62}$, inspired by the first spectrum of the water dimer by Miller63, has led to a very significant improvement in the understanding of these systems ${ }^{19}$. The method developed by Saykally is vibration-rotation-tunnelling spectroscopy carried out in the terahertz region of the electromagnetic spectrum, i.e. around $100 \mathrm{~cm}^{-1}$ (ref. ${ }^{19}$ ). In this spectral region, transitions are seen that involve not only rotations but also low-frequency vibrational and librational motions and, in particular, tunnelling motions. The water clusters all exhibit very significant tunnelling motions and the corresponding large-amplitude motions and passages 
through the associated transition states resemble complicated energy landscapes $^{64}$. A concerted effort by several theoretical groups from ab initio calculations by $X$ antheas $^{65}$ to full six-dimensional calculations by Leforestier ${ }^{66}$, earlier work of Kim and Jordan ${ }^{67}$, and the use of the diffusion Monte Carlo method by Clary 68,69 has now left us with a clearer picture of what is happening in water clusters and how their dynamics relate to the structure and dynamics of liquid water. The first conclusion that may be drawn is that when you build up the clusters to a certain size, the first three-dimensional structure is the hexamer, all other clusters are planar (or, more precisely, pseudo-planar because some hydrogens point out of plane) as can be seen in Fig. 4. Taking into account the ZPE, it is now believed that the cage structure for the water hexamer is the lowest in energy ${ }^{19 a}$, in contrast to earlier work which proposed the hexagonal structure. However, this might not yet be the final definitive answer for the hexamer structure since the full consideration of the zero-point energy level on such potentials of such high dimensionality is extremely demanding. In other words, for such systems entropy plays a most important role and it is necessary to consider not only the PES, but rather the free (Gibbs) energy surface (FES). With the present state-of-the-art it is now clear that the spectroscopy of water clusters and the corresponding theoretical efforts have led to very substantially improved potentials which will be useful for the simulation of bulk liquid water. These new potentials developed by different groups ${ }^{70,71}$ are based on

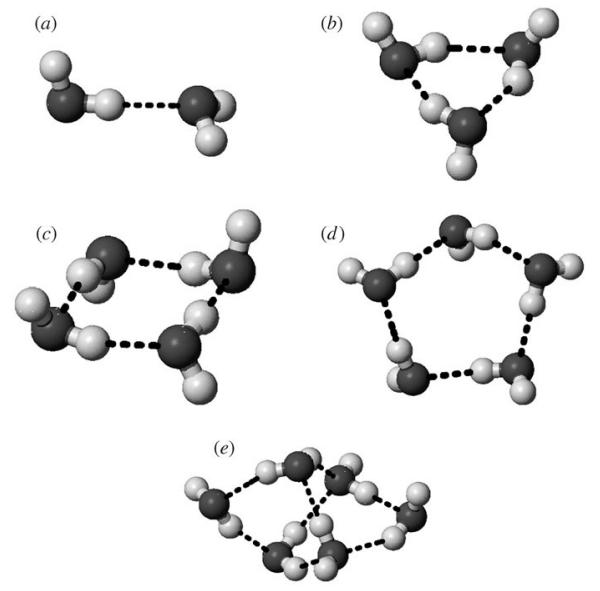

FIG. 4

Water cluster geometries, including the three-dimensional water hexamer structure. Reproduced from ref. ${ }^{19 a}$ with permission 
the knowledge gained from the cluster experiments: the pair potential must be known with extreme precision and the three-body interactions contribute about $20 \%$ to the potential energy; higher-body interactions give a rather negligible contribution. In the next few years we will see a very significant improvement in the modelling of the properties of liquid water from these results. In this context it should be mentioned that a completely different method for resolving the issue of modelling a complex hydrogen-bonded network such as water could come from the Carr-Parrinello method ${ }^{72}$. This method has been extensively tested on large complex systems and though it is based on density functional theory it is extremely powerful since the potential is calculated on the fly during the propagation of the dynamics, rather than using a static approximation.

\subsubsection{Rotational Coherence Spectroscopy}

Another, still quite new technique for the determination of rotational constants, using ultrafast laser pulses, is based on rotational coherence and the measurement of its recurrence ${ }^{73}$. Most recently, this method has been substantially refined by Brutschy, Riehn and co-workers ${ }^{74}$, using a two-colour, transform-limited picosecond laser system and ionization detection. Due to the very high quality of their spectra the analysis and the determination of rotational constants (which is not unambiguous) has been substantially improved and it is fair to state that rotational coherence spectroscopy is now a complementary method compared with energy level resolved spectroscopy ${ }^{74}$.

\subsubsection{Quantum Chemical ab initio Methods}

The 'inversion' of experimental data to obtain structure and geometry of a molecular cluster is normally not possible and fitting procedures using a suitable model are generally employed. In contrast, theoretical quantum chemical methods, using gradient optimization techniques, yield reliable structural and geometrical information even for extended complexes. Over the last half century, ab initio computational techniques have been developed and extensively used for covalent systems. The recipe for ab initio computations of non-covalent complexes is essentially the same: use basis sets as large as possible and take into account as much of the correlation energy as you can afford. For small complexes with no more than a few atoms, it is possible to optimize the geometry by using the most reliable (and most expensive) CCSD, CCSD(T) or even the CCSDT methods ${ }^{75}$. For 
extended complexes the use of these higher-level coupled cluster methods for geometry optimization is difficult and too expensive; as shown in ref. ${ }^{76}$, the coupled cluster methods do not give a very different geometry compared with MP2. The choice of basis set is critical for a reasonably reliable description of any structural type of non-covalent complex: the basis sets required should have at least two sets of first polarization functions and one set of second polarization functions, conditions satisfied by the cc-pVTZ basis set (for atoms of the second period and hydrogen: $[4 s 3 p 2 d 1 f / 3 s 2 p 1 d])$. A plethora of work provides strong evidence that the MP2/cc-pVTZ gradient optimization yields correct geometries for the neutral ground state of molecular complexes and that MP2/cc-pVTZ is the level of theory required to distinguish between, for instance, stacking and hydrogen bonding in nuclear base pairs. However, for extended complexes the MP2/cc-pVTZ level of theory is computationally challenging and becoming increasingly impractical with increasing size of the complex. Recently we explored $^{77}$ the applicability of the resolution of identity MP2 (RI-MP2) approximation method 78,79 . We have shown that when combined with extended basis sets containing f-functions, RI-MP2 is capable of accurate descriptions of $\mathrm{H}$-bonded and stacked DNA base interactions. The RI-MP2 method implemented in the TURBOMOLE code ${ }^{80}$ yields almost identical absolute as well as relative (interaction) energies as the exact MP2 method for the nucleic acid bases and base pairs studied ${ }^{77}$, whilst the computational time saving can be as large as one order of magnitude.

The reliability of the RI-MP2/TZVPP method (the TZVPP basis set (for atoms of the second period and hydrogen: [5s3p2d1f/3s $2 p 1 d]$ ) is practically identical with the Dunning's cc-pVTZ basis set) was tested for the phenol dimer $^{81}$ for which experimental rotational constants determined by time-resolved rotational coherence spectroscopy are available ${ }^{81}$. The optimized structure of the dimer is shown in Fig. 5 indicating that this dimer is

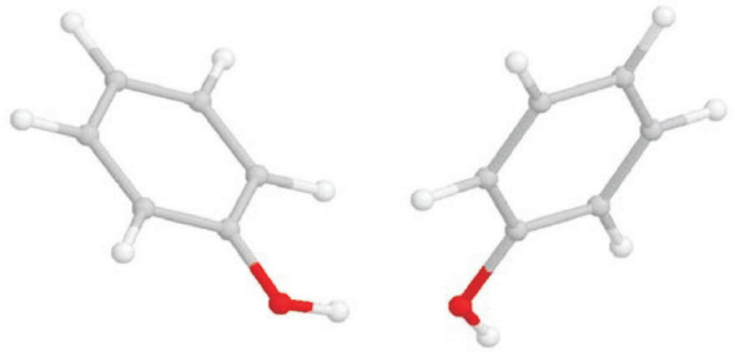

FIG. 5

Optimized structures of phenol dimer. Reproduced from ref. ${ }^{81}$ with permission 
not only stabilized by $\mathrm{OH}$...O hydrogen bonding but also by substantial interaction of the benzene rings. The correct description of such a structure thus requires not only a description of the $\mathrm{H}$-bond, which is not so particularly demanding, but also the description of the $\pi-\pi$ interaction, which is much more involved. Excellent agreement between experimental and theoretical rotational constants (with an average relative deviation from experimental value smaller than $1.5 \%$ ) was obtained. This gives us the confidence to propose the use of the RI-MP2/TZVPP method for the evaluation of complex geometries. When performing the calculations at the MP2/6-31G** level (this level is frequently used for complexes of this size), the relative deviation increased three times (to $4.5 \%$ ). Presently, we have no reason to doubt the reliability of the RI-MP2/TZVPP procedures for molecular clusters.

\subsubsection{Gradient Optimization and Basis Set Superposition Error}

The use of the gradient optimization techniques for non-covalent complexes is associated with a serious problem: the basis set superposition error $^{48}$ is normally not a priori included in the geometry optimization cycles. In contrast, the BSSE is mostly included a posteriori in order to improve the determination of stabilization energy. So, normally, this important correction is only taken into account when determining the stabilization energy but it is ignored in the geometry optimization of the complex. Only several years ago, a counterpoise-corrected gradient optimization procedure was introduced, which covers the basis set extension effect in each optimization cycle 82,83 . Until very recently, numerical applications were limited to small and medium basis sets as well as to structural motifs where the BSSE is rather small. Recently, we have used the counterpoise-corrected gradient optimization procedure for the phenol dimer ${ }^{81}$ : it was found that the relative deviation of rotational constants from experimental values was the same as in the case of standard MP2 gradient optimization, which we believe was due to the use of a small basis set. To test the procedure further we optimized the structure of stacked and planar $\mathrm{H}$-bonded uracil dimers and the stacked adenine...thymine pair ${ }^{76}$; besides the counterpoise-corrected gradient optimization, we also optimized the geometry of these clusters with a step-by-step procedure based on the CCSD(T) method, including the counterpoise correction in every step. The results can be summarized as follows: i) standard geometry optimization with small basis sets (e.g. 6-31G**) provides fairly reasonable intermolecular separation; ii) geometry optimization with extended basis sets at the MP2 level underesti- 
mates the intermolecular distances compared with the reference CCSD(T) results, whereas the MP2/cc-pVTZ counterpoise-corrected optimization agrees well with the reference geometries and, therefore, is recommended.

Geometrical data obtained from quantum chemical calculations corre spond normally to optimized geometries at stationary points on the PES. This data cannot be directly compared with experiment since experimental characteristics involve the vibrational energy. Even at $0 \mathrm{~K}$, the effect of the zero-point energy, to which all vibrations contribute, has to be considered. In order to compare to experiment, vibrationally averaged geometries have to be obtained around the stationary points on the PES. The spectacular effect of vibrational averaging was already demonstrated for the ammonia dimer in Section 2.2.1. In general, the less harmonic the PES is around a stationary point, the more pronounced is the effect of this vibrational averaging. When considering the zero-point vibrational energy level for very flat potentials, for which the harmonic approximation becomes meaningless, the position of the zero-point energy level can be rather difficult to estimate. For instance, for very flat potentials with two or more (adjacent) minima it is quite possible for the ZPE level to lie above a barrier.

The optimization procedure described above concerns rigid systems where the concept of structure is meaningful. In the case of floppy complexes, large-amplitude motions play a significant role such that the concept of a definite structure must be replaced by the more generalized concept of the potential energy surface (see Section 3.1. and the discussion of the ammonia dimer in Section 2.2.1.).

\subsection{Stabilization Energy}

We define stabilization energy as the negative of the dissociation energy measured from the dissociation asymptote to the minimum of the PES. The determination of stabilization energies is of key importance since it relates to fundamental thermodynamics. Stabilization energy can be only obtained from theoretical calculations, while experiments yield stabilization enthalpy (this corresponds to the inclusion of the zero-point energy). For experiments at very low temperatures (e.g. in a supersonic expansion molecular beam), the data refer to the stabilization enthalpy at $0 \mathrm{~K}$, which is obtained theoretically by adding the zero-point energy to the internal energy. However, for higher temperatures increases, the inclusion of temperature-dependent enthalpy terms, derived from the partition function, is required. 
Experimental determination of the stabilization enthalpy of a cluster is difficult and relevant data exists only for few complexes. The classical method involves measuring the temperature dependence of the equilibrium constant, which yields the stabilization enthalpy of complex formation. Using the field-ionization mass spectrometry method, Sukhodub et al. ${ }^{84}$ measured stabilization enthalpies of methylcytosine...methylcytosine, methylguanine...methylcytosine, methyladenine...methylthymine and methylthymine...methylthymine complexes. It must be stressed that these, almost 25 years old data are still the only data on stabilization energies (enthalpies) of DNA base pairs in vacuo.

\subsubsection{Mass-Resolved ZEKE Spectroscopy}

The best method so far to determine stabilization energies with spectroscopic precision comes from ZEKE spectroscopy ${ }^{9-11}$. The ZEKE spectroscopy method has been extensively reviewed ${ }^{85-95}$, also in the context of Rydberg state $^{96}$ and photoionization dynamics ${ }^{97}$ and applied to molecular clusters ${ }^{98}$. Let us recall that ZEKE spectroscopy is based on the pulsed field ionization of very long-lived Rydberg states ${ }^{9 d, 99,100}$ of very high principle quantum numbers ( $n>200$ ). The pulsed field ionization of a ZEKE Rydberg state produces both an electron and an ion. When this electron is measured we call this method zero electron kinetic energy (ZEKE) photoelectron spectroscopy; when the corresponding ion is selectively measured, we call this mass-selected ZEKE, or mass-analyzed threshold ionization (MATI)10,101-103. Since the motion of the Rydberg electron is completely decoupled from the internal motion of the ion core, a ZEKE spectrum results in the observation of vibrational and rotational structure of the corresponding molecular cation. This is fully equivalent to the spectrum one would obtain from photoelectron detection if one could improve the resolution to the required level. Mass-selected ZEKE provides the additional advantage of having a mass signature, which is particularly useful for molecular clusters and the observation of fragmentation processes. For molecular clusters, the most useful application of MATI comes from observation of the dissociation of the Rydberg state ion core which allows the determination of dissociation and binding energies of the molecular cluster cation. Once the cation dissociation energy is known, the dissociation energy of the neutral cluster is also known, as we have shown for the phenol... $\mathrm{N}_{2}$ and phenol...CO complexes $^{104}$ and for other complexes ${ }^{105}$. In terms of spectral resolution, the MATI resolution can be better than $0.1 \mathrm{~cm}^{-1}$ (ref. ${ }^{103}$ ), and ZEKE and MATI are fully equivalent ${ }^{10,106}$. For the phenol...argon complex, Fig. 6 shows a 
comparison between ZEKE electron (a) and MATI detection with high (b) and lower (c) spectral resolution. The insert in Fig. 6 (b) shows an enlarged section of both the phenol ${ }^{+} . .$. Ar (parent) and the phenol ${ }^{+}$(daughter) ion around the dissociation threshold of the phenol ${ }^{+} . .$. Ar cluster. It can be seen that the dissociation energy can be very precisely determined as the difference between the disappearance of the parent ion and the appearance of the daughter fragment ion. The corresponding energetics for the cation ground state, the neutral excited state and the neutral ground state are illustrated in Fig. 7 along the intermolecular dissociation reaction coordinate.

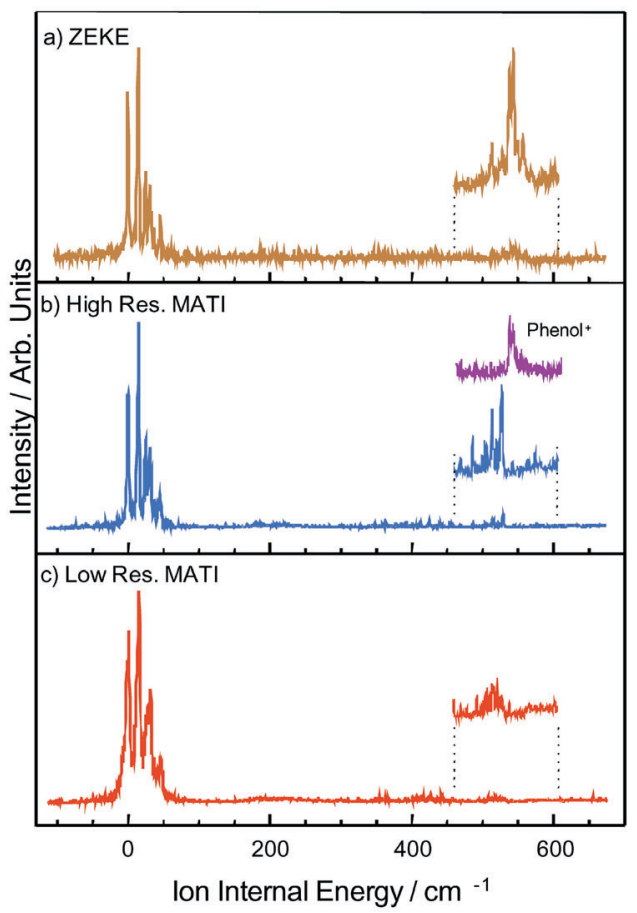

FIG. 6

ZEKE spectroscopy of phenol...argon: (a) High-resolution ZEKE spectrum (electron detection); (b) high-resolution and (c) low-resolution MATI spectra measured for the phenol ${ }^{+}$...argon (parent) and phenol ${ }^{+}$(daughter) dissociation product. The dissociation threshold is enlarged in the inserts in (b) and (c). The dissociation energy can be very precisely determined in (b) at the point of disappearance of the parent ion and the appearance of the daughter fragment ion. $\mathrm{Re}$ produced from ref. ${ }^{10}$ with permission 


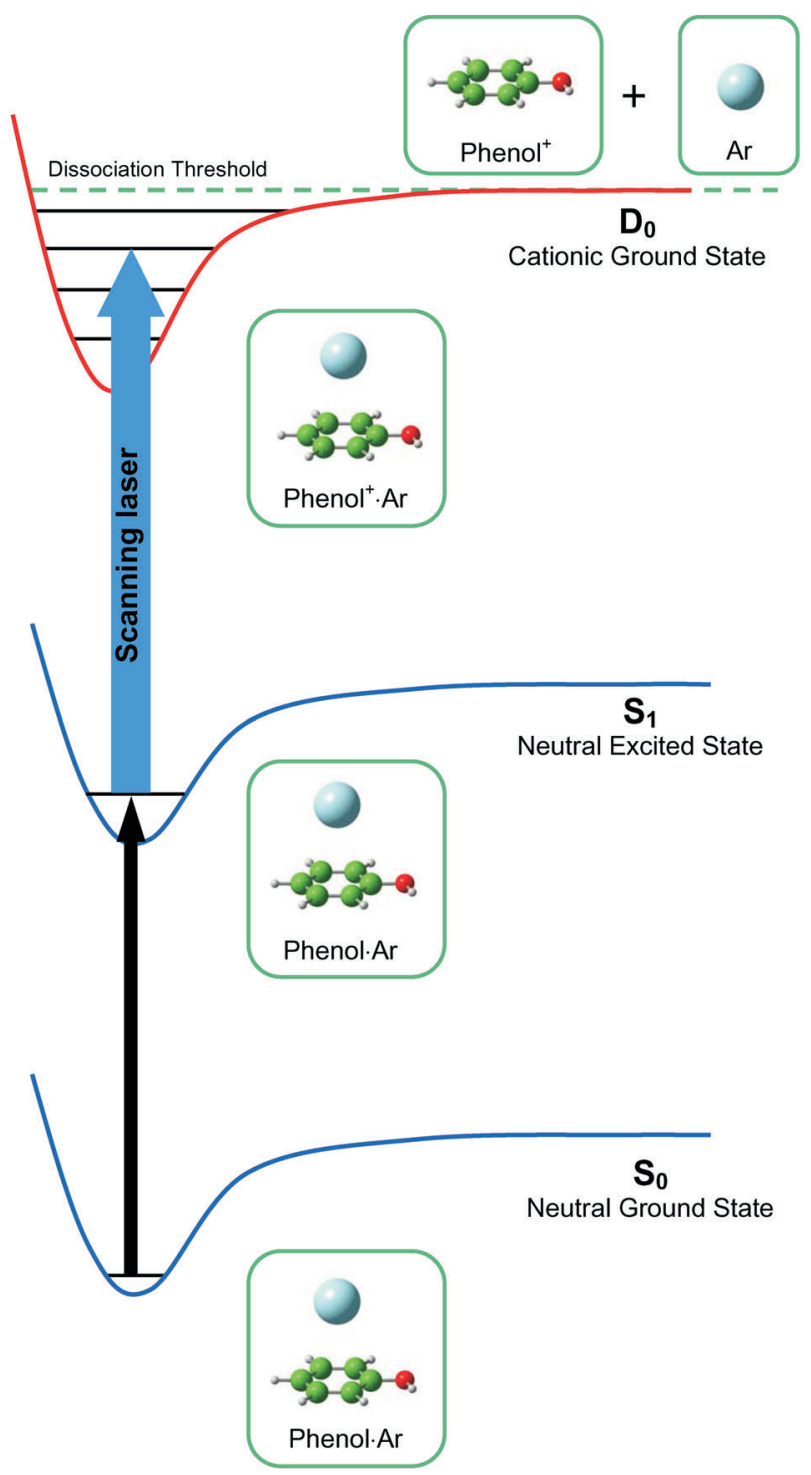

FIG. 7

Energy diagram for the MATI dissociation process 
The way that this high-precision dissociation spectroscopy works is depicted in Fig. 8. The ZEKE Rydberg electron circles around the ion core at a very large distance. Any dynamic process, such as vibrational predissociation, even electronic excitation in the cation in the core, will leave the Rydberg electron untouched. So if the ion core dissociates, the ionic fragment will continue to support the Rydberg electron whereas the neutral fragment will escape virtually unnoticed. Upon pulsed field ionization the ion generated will then be the daughter fragment ion and no longer the parent ion. In the time of flight spectrum, the daughter ion will be measured and if this is done simultaneously with the measurement of the parent ion then a spectrum such as that shown in Fig. 6 will be obtained. By invoking a Haber-Bosch cycle, the dissociation energy in the ionic complex can then immediately be used to determine the dissociation energies in the neutral excited state $\left(\mathrm{S}_{1}\right)$ and/or the neutral ground state $\left(\mathrm{S}_{0}\right)$. It cannot be stressed enough that presently the only viable method of high spectroscopic precision for the determination of dissociation and stabilization energies of molecular complexes comes from this mass-resolved variant of ZEKE spectroscopy.

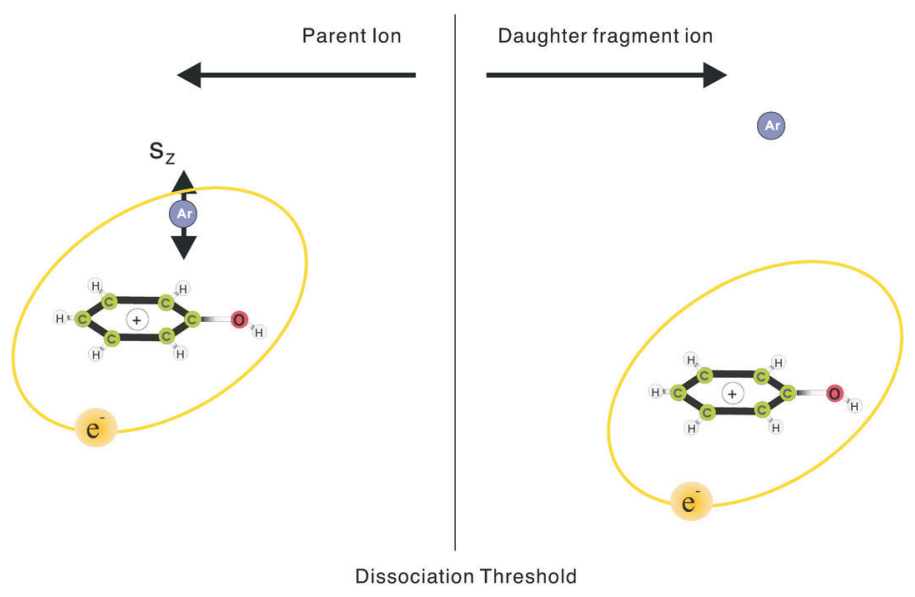

FIG. 8

Dynamics of the MATI dissociation process. The ZEKE Rydberg electron is not perturbed by any dynamic process, including vibrational predissociation of the ion core. When the ion core dissociates, the ionic fragment will continue to support the Rydberg electron due to the dominant Coulomb interaction. Upon pulsed field ionization, the ion produced is the (daughter) fragment ion. The neutral fragment is not detected 


\subsubsection{Computation of Stabilization Energy}

Compared with the determination of a geometry, the theoretical determination of stabilization energies requires using higher-level techniques (in comparison with the determination of a structure) since the stabilization energy is more sensitive to accurate description than the structure itself. First, it is not correct to limit the calculations solely to the MP2 level; higher correlation energy contributions should be included. It is thus not recommendable to use DFT techniques since they do not cover a sufficient part of the correlation energy. Specifically, the London dispersion forces are not covered if any of presently used DFT functionals are adopted. The CCSDT method, considering single, double and triple excitations (as well as part of higher excitations) iteratively (up to the infinite perturbation order), is still limited to complexes with less than 10 atoms. Only very recently, the first study appeared where the stabilization energies of model $\mathrm{H}$-bonded and stacked systems were determined at the CCSDT level ${ }^{107}$. A compromise between economy and accuracy is the $\operatorname{CCSD}(T)$ method where the triple excitations are covered in a non-iterative way. Because of the strong dependence of stabilization energy on the AO basis set size, it is important to perform the calculation with as a large basis set as possible or, preferably to do it at the complete basis set (CBS) limit. The determination of a CBS limit of the $\operatorname{CCSD}(T)$ calculations is impractical since we cannot obtain two energy points generated with systematically improved $A O$ basis sets. Let us mention that the first reliable basis set (in the cc-pVXZ series) is the Dunning aug-cc-pVDZ basis, and then aug-cc-pVTZ ${ }^{108}$. The CCSD(T) calculations for complexes mentioned are impractical for basis sets larger than $\mathrm{DZ}+\mathrm{P}$ (e.g. 6-31G** or cc-pVDZ). There exists, however, a single method by which one may overcome this problem. This is based on the fact that $\operatorname{CCSD}(\mathrm{T})$ and MP2 energies have a similar dependence on the size of basis set used (cf. Fig. 9). Assuming the difference between CCSD(T) and MP2 interaction energies $\left(\Delta \mathrm{E}^{\mathrm{CCSD}(\mathrm{T})}-\Delta \mathrm{E}^{\mathrm{MP2}}\right)$ exhibits a relatively small basis set dependence compared with MP2 CBS determination, the CBS CCSD(T) interaction energy can be approximated as

$$
\Delta \mathrm{E}^{\mathrm{CCSD}(\mathrm{T})}{ }_{\mathrm{CBS}}=\Delta \mathrm{E}^{\mathrm{MP2}} \mathrm{CBS}+\left.\left(\Delta \mathrm{E}^{\mathrm{CCSD}(\mathrm{T})}-\Delta \mathrm{E}^{\mathrm{MP2}}\right)\right|_{\text {medium basis set }} \cdot
$$

The CCSD(T)-MP2 difference has been investigated for $\mathrm{H}$-bonded as well as stacked model complexes ${ }^{46}$. It was found that even rather small basis sets like cc-pVDZ $(0.25,0.15)$, where the exponents of $d$ - and p-polarization 
functions were equal to 0.25 and 0.15 to ensure a better description of stacked complexes, yield satisfactory values for this difference (cf. Fig. 9).

The MP2 part of the stabilization energy is extrapolated to the CBS limit as follows. Whereas the HF interaction energy can be considered to converge with respect to the one-electron basis set al ready for relatively small basis sets, the MP2 part of the interaction energy converges to its complete basis set limit unsatisfactorily slowly (cf. Fig. 9). In order to correct the computed results for the basis set incompleteness error, several extrapolation schemes have been successfully employed in literature. This is the scheme of Helgaker and co-workers ${ }^{109}$ :

$$
E_{X}^{H F}=E_{C B S}^{H F}+A e^{-\alpha X} \quad \text { and } \quad E_{X}^{\text {corr }}=E_{C B S}^{\text {corr }}+B^{-3}
$$

and of Truhlar ${ }^{110}$ :

$$
E_{X}^{H F}=E_{C B S}^{H F}+A X^{-\alpha} \quad \text { and } \quad E_{X}^{H F}=E_{C B S}^{H F}+B X^{-\beta}
$$

where $E_{X}$ and $E_{C B S}$ are energies for the basis set with the largest angular momentum $X$ and for the complete basis set, respectively; $\alpha$ and $\beta$ are parameters fitted by the authors ${ }^{109,110}$. These schemes were chosen because (i) both

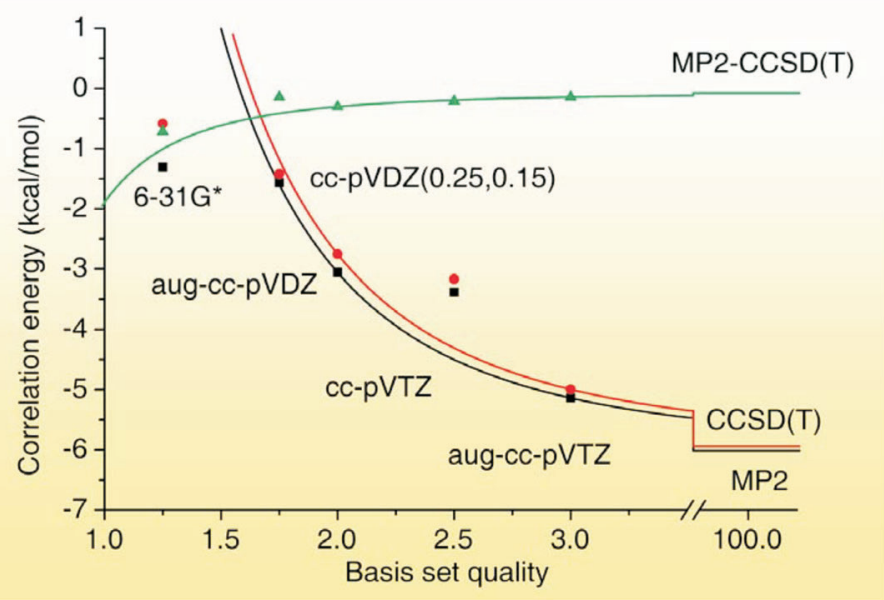

FIG. 9

The dependence of MP2, CCSD $(T)$ and $(M P 2-C C S D(T))$ interaction energies on the basis set size (2.0, 3.0 and 100.0 mean double-zeta, triple-zeta and CBS limit, respectively). Reproduced from ref. ${ }^{46}$ with permission 
approaches extrapolate HF and correlation energy separately and (ii) both use the two-point form (they extrapolate two successive basis sets results). The two-point extrapolation form is preferable as it was shown ${ }^{111}$ that inclusion of additional (lower-quality basis set) results in the extrapolation often spoils the quality of the fit, especially when the smallest basis set (cc-pVDZ) is used. For non-covalent complexes it is recommended to use augmented Dunning's basis sets rather than non-augmented ones to reduce the extrapolation error (note that the aug-cc-pVDZ basis set gives absolute energies as well as interaction energies comparable with those calculated with the TZVPP basis).

Extrapolation to the complete basis set limit is also important from the point of view of the basis set inconsistency problem. The BSSE gives rise to a better description of the supersystem compared with the subsystems; the supersystem uses basis sets of both subsystems (or all subsystems in more extended species), forming the larger dimmer-centred basis set (DCBS). The BSSE is largest for small basis sets and its value reduces when the basis set size increases. When working with infinite basis sets, the BSSE should converge, by definition, to zero. Figure 10 shows the dependence of stabilization energies of the adenine...thymine pair on the AO basis set size. It is evident that corrected as well as uncorrected stabilization energies converge to the same complete basis set limit, the first from above while the latter from below (though, in the second instance, not necessarily in a

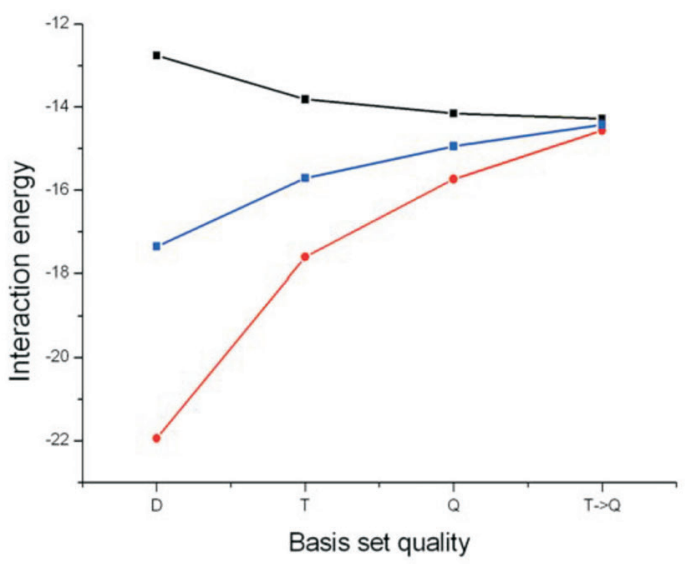

FIG. 10

Dependence of uncorrected (red line) and BSSE-corrected (blue and black lines refer to inclusion of $50 \%$ and $100 \%$ of BSSE) stabilization energies (energies in $\mathrm{kcal} / \mathrm{mol}$ ) of hydrogen-bonded structure of the adenine...thymine pair on the AO basis set size ( $T \rightarrow Q$ means CBS limit obtained from extrapolation of aug-cc-pVTZ and aug-cc-pVQZ energies) 
monotonic fashion). From Fig. 10 it is further evident that when using small and medium AO basis sets, the corrected stabilization energy is closer to the CBS limit and as such justifies the use of BSSE corrections when working with these basis sets.

Recently, numerous applications of extrapolation techniques for small systems have been described and reviewed ${ }^{112}$. This is illustrated by two earlier ${ }^{113 a, 113 b}$ and a few more recent works ${ }^{113 c-113 f}$. Equilibrium structures and energetics for two minima and an activated complex at the Ar...HF PES (minima: Ar...HF and Ar...FH) were calculated with the BSSE taken into consideration during gradient optimization ${ }^{113 a}$. The CBS MP2 value for water dimerization was ascertained ${ }^{113 b}$ to be $-4.94 \pm 0.02 \mathrm{kcal} / \mathrm{mol}$. A vdW radical, $\mathrm{HeBr}$, and a vdW molecule, $\mathrm{HeBr}_{2}$, were studied with basis sets of double through quintuple zeta quality (i.e., $X=2,3,4,5$ ). In addition, potential energy surfaces together with transport, scattering and spectroscopic properties were obtained ${ }^{113 c}$. For simple vdW molecules $\left((\mathrm{Ne})_{2},(\mathrm{Ar})_{2}\right.$, $(\mathrm{HF})_{2}$ and $\left.\left(\mathrm{H}_{2} \mathrm{O}\right)_{2}\right)^{113 d}$, acetylene dimer ${ }^{113 e}$, and for cations $\left(\mathrm{H}_{3}+\ldots \mathrm{Rg}\right)$, molecules $\left(\left(\mathrm{H}_{2}\right)_{2}\right)$, and anions $\left(\mathrm{CH}_{4} \ldots \mathrm{H}^{-}\right)^{113 f}$, CBS energies and structural features were obtained through plots of those characteristics against reciprocal values of the number of functions in the respective basis set.

The performance of the above mentioned theoretical procedure for determination of structure and stabilization energy was tested for the benzene...indole complex ${ }^{114}$ for which the stabilization enthalpy is known from the MATI experiments. As mentioned before, the technique does yield an experimental stabilization enthalpy but does not provide any information on the structure of a complex. We considered therefore two possible arrangement of the dimer, a stacked structure (a) and an $\mathrm{N}-\mathrm{H} . . . \pi \mathrm{H}$-bonded structure (b). In the first step, both structural motifs were optimized at the RI-MP2/TZVPP Ievel; the optimized geometries are presented in Fig. 11. The

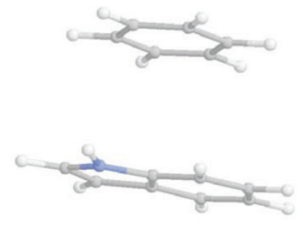

a

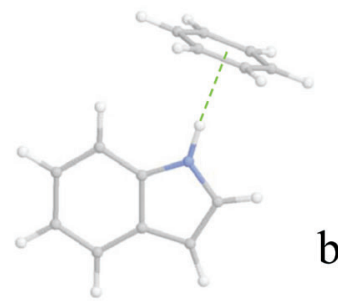

FIG. 11

Stacked (a) and N-H... $\pi$ hydrogen-bonded structure (b) of the benzene...indole dimer. Reproduced from ref. ${ }^{114}$ with permission 
RI-MP2 stabilization energy of the stacked structure was slightly larger than that of the $\mathrm{H}$-bonded structure $(6.57$ and $6.20 \mathrm{kcal} / \mathrm{mol})$ and this difference was further enlarged when passing to stabilization enthalpies at $0 \mathrm{~K}$ (the ZPE was added). Performing the $\operatorname{CCSD}(T)$ calculations with a 6-31G** $(0.25$, 0.15 ) basis set, the preference of both structures was reversed and the $\mathrm{N}-\mathrm{H} . . . \pi \mathrm{H}$-bonded structure becomes more stable $(4.24$ and $2.81 \mathrm{kcal} / \mathrm{mol})$. This is a remarkable effect giving clear evidence for the role of higher correlation energy terms. Evidently, when comparing stabilization energies of various structural motifs, the higher-order correlation energy contributions should be properly covered. Finally, the CBS limit of the M P2 stabilization energy was determined for the global minimum and putting together all the contributions we obtained an estimate of the true stabilization enthalpy of the $\mathrm{N}-\mathrm{H} \ldots \pi \mathrm{H}$-bonded structure $(5.3 \mathrm{kcal} / \mathrm{mol})$. This value nicely agrees with the experimental estimate $5.2 \mathrm{kcal} / \mathrm{mol}$ and enhances confidence in the use of this procedure for other extended non-covalent complexes. Let us finish, however, by saying that further testing of the procedure is necessary which requires more extensive sets of experimental data.

\subsection{Is Density Functional Theory (DFT) Capable of Describing Non-C ovalent Interactions?}

The answer is simple: In cases where the contribution of dispersion energy is significant or even dominant, DFT is not applicable or otherwise unsuitable to this kind of calculation ${ }^{115}$. This concerns not only the interaction of rare atoms, but also selected interaction types of nucleic acid bases and amino acids. In numerous other instances DFT works well ${ }^{116}$. This concerns geometry and energetics of $\mathrm{H}$-bonded complexes, geometry and spectral features of classic charge-transfer complexes ${ }^{116 a}$, the magnetic exchange coupling between transition-metal ions in dinuclear complexes ${ }^{116 b}$, metal ligand aromatic cation- $\pi$ interactions (topical for example in metalloproteins ${ }^{116 c}$ ) and NMR properties of xenon-containing compounds (interaction energies, however, were evaluated by the MP2 ab initio method $^{116 f}$ ). Finally, matrix-isolation experiments (IR) with complexes between $\mathrm{CO}$ and a set of dihalogen molecules were carried out and results were discussed on the basis of DFT calculations.

Attempts associated with overcoming this inherent weakness were made along several lines ${ }^{117}$. Ab initio DFT was developed and its relationship to coupled-cluster methods analyzed ${ }^{117 a-117 c}$. In contrast to the standard DFT (local, gradient-corrected, or hybrid), the ab initio version correctly de- 
scribes London interaction in, for example, $(\mathrm{He})_{2}$ and $(\mathrm{Ne})_{2}$. The DFT approach was also used for calculations of potential energy surfaces of van der Waals complexes ${ }^{117 d, 117 e}$.

Some deficiencies of the symmetry-adapted perturbation treatment, SAPT (Kohn-Sham), were overcome by using an asymptotic correction. This concerns the electrostatics, the first-order exchange, the second-order induction and exchange-induction ${ }^{117 d}$ energies. The remaining difficulty concerned dispersion energy. By means of a generalized Casimir/Polder formula (evaluated with dynamic density susceptibilities obtained from TD DFT), very good and economically acceptable results were obtained ${ }^{117 e}$ for $(\mathrm{He})_{2},(\mathrm{Ne})_{2}$, and $\left(\mathrm{H}_{2} \mathrm{O}\right)_{2}$. The results obtained for a weakly bound complex ${ }^{117 f}$ using the Perdew-Wang exchange and Perdew-Wang correlation terms (PW 91PW91) combined with Adamo and Barone's Becke-style one-parameter functional are promising, but more testing is necessary. However, the only generally acceptable DFT technique which covers dispersion energy is a combination of DFT and London dispersion energy ${ }^{116 d}$. This semiempirical method (combination of tight binding DFT and empirical dispersion correction) gives very promising results comparable with accurate correlated results for $\mathrm{H}$-bonded as well as stacked nucleic acid base pairs and various motifs of amino acid base pairs ${ }^{118}$.

\subsection{Vibrational Frequencies}

A very important feature of vibrational frequencies is the fact that they are observable and the formation of a non-covalent complex can be easily detected by measuring its intermolecular vibrational frequencies. Upon formation of a non-covalent complex, new intermolecular frequencies arise, which are generally much smaller than intramolecular frequencies and are typically observed in the sub- $100 \mathrm{~cm}^{-1}$ region of the vibrational spectrum; frequently, they are below $50 \mathrm{~cm}^{-1}$. Generally, six intermolecular frequencies exist, corresponding to the loss of three translational and three rotational degrees of freedom upon formation of a complex. Intermolecular frequencies are mostly rather similar for various types of non-covalent complexes and thus cannot be used for identification of a specific complex. For the identification, one can use changes of intramolecular frequencies upon formation of a complex, the observed shift of which usually correlates with the strength of molecular interactions. Well known is the shift of $\mathrm{X}-\mathrm{H}$ stretching frequencies to lower values upon formation of a hydrogen bond of the $\mathrm{X}-\mathrm{H}$...Y type. This red shift can be very large, even several hundreds of wavenumbers affording an easy and unambiguous way of proving the 
formation of an $\mathrm{H}$-bonded complex. In the recent years an opposite shift, i.e. a shift to higher frequencies (blue shift), was also observed for a similar class of systems and the so-called improper blue shifting $\mathrm{H}$-bond was detected in many complexes in nature. More detailed information on both types of $\mathrm{H}$-bonding will be provided later.

Harmonic frequencies are easily determined even for large non-covalent cluster using the Wilson FG analysis and the procedure is now routinely available in quantum chemical codes. The frequencies calculated, however, are in reality non-harmonic, and the effect of anharmonicity should be taken into consideration. Mostly this is covered via scaling and there exist recommended values of scaling factors, which are different for Hartree-Fock and correlated calculations. The use of scaling brings without doubt better agreement between experimental and theoretical values but its use cannot be generally recommended since it sometimes leads to deterioration of this agreement. The standard approach to the non-harmonic vibrational problem for large non-covalent clusters is the perturbation theory ${ }^{119,120}$. If the zero-order Hamiltonian (usually a harmonic oscillator) is a good approximation to the true vibrational Hamiltonian, the perturbation theory is a very efficient and reliable tool for calculating vibrational frequencies. In the traditional approach, the matrix representation of the molecular Hamiltonian is diagonalized by successive contact transformations. This procedure, however, fails in the case of accidental resonance, which is often the case if we deal with large systems with many vibrational modes. In this case the terms connecting the resonant levels have to be treated variationally ${ }^{120,121}$ and considerable progress was achieved in developing new procedures based on perturbative treatment for the calculation of anharmonic frequencies ${ }^{121-123}$. The potential energy function is constructed as a low-order polynomial (up to the fourth order) expressed in normal coordinates. The force constants are obtained by least-squares fitting of energies, gradients, and Hessians calculated at geometries close to the global minimum on the PES. The main advantage of this approach stems from its computational efficiency as the number of the required Hessians scales linearly with the number of vibrational modes. Thus, the method can even be used for large systems while respecting the full dimensionality of the problem. However, the applicability of the procedure is less straightforward for non-covalent clusters since they are non-rigid systems. The vibrational dynamics of floppy systems cannot be described in the framework of a single-reference Hamiltonian and, therefore, the perturbation series used are necessarily strongly divergent. In such a case the only alternative is a more exact treatment of the large-amplitude vibrational 
modes including all relevant parts of the coordinate space. This requires calculation of the global PES, which becomes computationally prohibitive even for systems with only a few degrees of freedom. However, the number of large-amplitude motions is usually a small fraction of the total number of vibrations. Consequently, the large-amplitude vibrations can be removed from the perturbative treatment and the Schrödinger equation for the effective large-amplitude Hamiltonian solved variationally. However, a coupling between large-amplitude vibrational mode and other modes is not considered and can lead to rather large errors.

The fully variational method is free of any limitation but it is prohibitively expensive even for problems of low-dimensionality. Literature on higher-dimensional anharmonic vibrational calculations of non-covalent clusters based on ab initio correlated calculations includes a variational six-dimensional intermolecular vibrational frequency calculation for the adenine...thymine Watson-Crick base pair ${ }^{124}$ and a twelve-dimensional vibrational frequency calculation for the water dimer by perturbation theory ${ }^{125}$. Also, a six-dimensional frequency calculation for the water dimer based on various empirical potentials was reported recently by LeForestier et al. ${ }^{66}$ Other rigorous treatments of vibrations in clusters have al so been reported ${ }^{126-129}$. Harmonic and anharmonic vibration frequencies were determined for the guanine...cytosine complex and compared with gas-phase IR-UV double resonance spectral data. Harmonic frequencies were obtained at RI-MP2/cc-pVDZ and RI-MP2/TZVPP levels and anharmonic frequencies were obtained by the CC-VSCF method based on improved semiempirical PM 3 results ${ }^{130}$. Comparison of the data with experimental results indicates that the average absolute percentage deviations for the method is $2.6 \%$ for harmonic RI-MP2/cc/pVDZ, 2.5\% for harmonic RI-MP2/TZVPP and 2.3\% for the adopted PM 3 CC-VSCF. The use of empirical scaling factor for the $\mathrm{ab}$ initio harmonic calculations improves the stretching frequencies but decreases accuracy of the other mode frequencies ${ }^{130}$.

The soft intermolecular vibrational modes may serve for structural elucidation. Their total number ranges from one ( $v d W$ system consisting of two atoms) up to six, which is available for any vdW system consisting of two polyatomic subsystems. The hydrogen fluoride dimer possesses four intermolecular modes for obvious reasons. The vibrational predissociation $^{131 a}$ and the vibrational second overtones ${ }^{131 b}$ of $(\mathrm{HF})_{2}$ were studied thoroughly. Counterpoise-corrected ab initio analytical potential energy and dipole hypersurfaces were computed and the dimer dissociation energy, $D_{e}=19.1 \mathrm{~kJ} / \mathrm{mol}$, was obtained ${ }^{131 c}$. The energy barrier to the hydrogen bond exchange amounts to $4.2 \mathrm{~kJ} / \mathrm{mol}$; the disrotatory in-plane bending vi- 
bration involved in this process was studied earlier ${ }^{131 d}$. A careful study of near infrared spectra of $(D F)_{2}$ permitted determination of all four intermolecular modes ${ }^{131 e}$. Extensive attention was paid to the role of the basis set superposition error (BSSE) in connection with oligomers $(H F)_{n}(n=3,4)$; the consequences are of greater importance ${ }^{131 f}$.

The high-resolution $\mathrm{IR}^{131 \mathrm{~g}}$ and vibrational excitation ${ }^{131 \mathrm{~h}}$ spectra were recorded and analyzed. An information on the coupling between the intraand intermolecular modes can be obtained from the red shift of the HF stretching mode; an analysis of the rotational constants ${ }^{131 \mathrm{~g}}$ can be used for the same purpose. The CCSD $(T)$ calculations were carried out in a study of vibrational predissociation of the $\mathrm{Ne} . . \mathrm{Br}_{2}$ system ${ }^{131 i}$. High-quality PES for $\left(\mathrm{N}_{2}\right)_{2}$ lead $^{131 j}$ to a T-shaped structure with a well depth of $107 \mathrm{~cm}^{-1}$ and a distance of $4.03 \AA$. A rotationally resolved IR spectrum for $\mathrm{C}_{2} \mathrm{H}_{4} \ldots \mathrm{HCl}$ (with ${ }^{35} \mathrm{Cl}$ and ${ }^{37} \mathrm{Cl}$ ) was interpreted with the assistance of the CCSD(T) calculations ${ }^{131 k}$.

A $\pi$-type complex between 2,3-dihydrobenzofuran (coumaran) and Ar was studied by combining resonance-enhanced multiphoton ionization and zero electron kinetic energy spectroscopy ${ }^{1311}$.

The $\mathrm{OH}$...CO vdW radical represents an intermediate in the $\mathrm{OH}+\mathrm{CO} \rightarrow \mathrm{H}+$ $\mathrm{CO}_{2}$ process, which assumes an important role in combustion and atmospheric chemistry ${ }^{132}$. Intermolecular excitations in the region $50-250 \mathrm{~cm}^{-1}$ were interpreted with the assistance of the CCSD(T) method.

Multiphoton IR photodissociation spectroscopy was used for the investigation of the solvation effect with the $(\mathrm{HBr})_{n} \mathrm{Br}^{-}(n=1,2,3)$ systems. A harmonic approximation is not sufficient for the interpretation of experimental IR spectra ${ }^{133 a}$. The intermolecular $\pi$-bond between protonized benzene $\left(\mathrm{C}_{6} \mathrm{H}_{7}^{+}\right)$and $\mathrm{Ar}, \mathrm{N}_{2}, \mathrm{CH}_{4}$, and $\mathrm{H}_{2} \mathrm{O}$ was studied by means of IR photodissociation spectra of mass-selected clusters and MP2 calculations ${ }^{133 b}$.

\section{POTENTIAL ENERGY AND FREE ENERGY SURFACES}

\subsection{Empirical Potentials and an Attempt to Pass to More and More} Extensive Systems: Combination of Various Elements of Theory

Empirical potentials continuously attract attention in the area of clusters of rare gas atoms as well as non-covalent species containing rare gas atoms and small molecules of various sorts. Examples come from the highly accurate semiempirical interatomic potential for argon clusters ${ }^{134}$ and from high-resolution spectroscopy, which served to obtain the potentials that 
permitted the determination of secondary minima for the Ar...HF ${ }^{135}$ and Ar...HCl ${ }^{136}$ non-covalent species.

During the last few years, increasing attention has been paid to empirical potentials for more complex systems, including water. The essential role of many-body contributions are well known for water molecule clusters; frequently, various potentials were used for the gas, liquid, and solid states. An effective pair potential was first introduced for all three phases ${ }^{137}$.

Monte Carlo and molecular dynamics simulations, in spite of an increasing tendency to use quantum chemical calculations, still frequently use empirical potentials; nowadays, however, a hybrid procedure based on a combination of quantum mechanical and molecular mechanical techniques has been widely used. Such a procedure makes it possible to obtain realistic values for hydration Gibbs energy, not only for a set of small molecules and ions, but also for acetic acid, glycine (in zwitterionic and non-zwitterionic forms) in water and for the $\mathrm{Ag}^{+} . .$. glycine complex ${ }^{138}$. An instructive review on sophisticated potentials suitable for MD treatment of biomolecules is available ${ }^{139}$.

\subsection{Nucleic Acid Base Pairs}

It is difficult to treat the PES of extended complexes containing large numbers of transition structures and energy minima separated by low-energy barriers ${ }^{64}$. Let us note here that the gradient optimization techniques localize the nearest energy minimum and then stop the calculation. On encountering such a stationary point (for which the gradient is zero by definition), which can be a minimum or, in fact, a saddle point, it is then necessary to restart the optimization from a different geometry and to hope that, after several trials, the whole PES will be sampled. It is true though that neither chemical intuition nor experience will necessarily be of any considerable help in elucidating a potential energy surface, especially in more complex systems. The use of an efficient sampling technique is thus inevitable and computer experiments offer an ideal solution. The aim is not only to localize the global minimum but also to identify all other energy minima, as a full description of the PES is of key importance for subsequent comparison of theory with experimental results. For this reason, the use of methods like simulated annealing, which aims to find global minima only, is of limited use. Instead, techniques like molecular dynamics (MD) simulations in combination with quenching techniques ${ }^{140}$ (MD/Q) are more useful. Variation of the kinetic energy (it should be higher than the energy of the highest transition structure) and the length of a quench ensure the proper sampling of 
the whole surface. In performing the longer MD simulations, one can obtain information about the population of various energy minima, which corresponds to the Gibbs energy change. Simulations can be performed in the NVT canonical or NVE microcanonical ensemble (N, V, E and T refer to the number of molecules in a system, its volume, energy and temperature, respectively). In the NVT canonical ensemble, the cluster is in thermal equilibrium with the surroundings and, accordingly, the NVT ensemble yields information about the behaviour of the cluster when it is interacting with the surroundings. In the NVE microcanonical ensemble, all systems have the same energy and each system is individually isolated. Performing simulations in either ensemble (depending on the type of experiment) allows to pass from the potential energy surface to the Gibbs energy surface. It is not surprising that entropy plays a different role for different types of molecular clusters and, thus, the potential energy surface and Gibbs energy surface can differ. Indeed, for the most parts the two surfaces are significantly different. Very frequently, the structure of a global minimum at the potential energy surface differs from that at the Gibbs energy surface.

The MD/Q technique allows to sample the surface, but the final description of the surfaces strongly depends also on the quality of the potential used. Despite the enormous progress of ab initio MD simulations, they are still limited to rather small systems; the size of DNA or RNA base pairs is still unattainable, being completely intractable for such procedures. The only chance for elucidation of such large systems is thus the use of empirical potentials. We must repeat that the quality of $\mathrm{MD} / \mathrm{Q}$ results is strongly affected by the quality of the potential and the use of an empirical potential that does not correctly describe the structure of a complex leads only to wrong and therefore misleading results. Thus, care should be taken in the choice of the potential, since not every potential used for simulations of DNA and RNA is also suitable for description of base pairs. We accumulated extensive evidence that the Cornell et al. potential ${ }^{141}$, prepared and parameterized in the Kollman laboratory, is well suited for these purposes. This evidence is based on comparison of structures and stabilization energies of a large number of DNA and RNA base pairs evaluated by this potential and by correlated nonempirical ab initio calculations ${ }^{142}$. Frequently we were surprised how well this potential (which is in fact rather simple and does not contain any features of the advanced potentials of the last generation, such as polarization terms or inclusion of higher than harmonic terms) describes various structural types of nucleic acid base pairs. Evidently, Kollman and his team were fortunate in the parameterization of 
this potential, which, due to compensation of many errors, describes nucleic acid bases and their complexes so accurately.

The MD/Q calculations were used intensively for study of potential energies and Gibbs energy surfaces of nucleic acid base pairs. First, individual base pairs were studied (uracil dimer ${ }^{143}$, the adenine...2,4-difluorotoluene pair $^{144}$, methyluracil dimers ${ }^{145}$, the adenine...thymine pair ${ }^{146}$ and the methyladenine...methylthymine pai ${ }^{147}$ ), and, later, all the DNA base pairs and methylated DNA base pairs were also considered ${ }^{148}$.

The complexity of the problem will be demonstrated on the case of the adenine...thymine (non-methylated) base pairs ${ }^{146}$. MD/Q calculations revealed twenty-seven energy minima, of which nine were $\mathrm{H}$-bonded, eight T-shaped and ten stacked (cf. Fig. 12). The H-bonded structures were the most stable ( $12 \mathrm{kcal} / \mathrm{mol})$, with stacked and T-shaped structures found to be less stable by at least $4 \mathrm{kcal} / \mathrm{mol}$. The global minimum and first two local minima surprisingly correspond neither to Watson-Crick nor to Hoogsteen structural types; the bonding is realized through $\mathrm{N}^{9}-\mathrm{H}$ and $\mathrm{N}^{3}$ functional
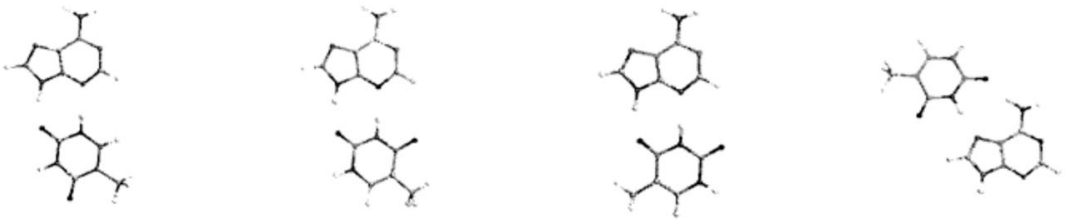

$\mathrm{p}(3192) ;-15.5 /-16.8 ; 29.1 \% \quad \mathrm{p}(3392) ;-14.2 /-14.3 ; 13.2 \%$

$\mathrm{p}(3394) ;-14.0 /-14.4 ; 12.2 \%$

$\mathrm{p}(6273) ;-13.0 /-12.6 ; 8.5 \%$
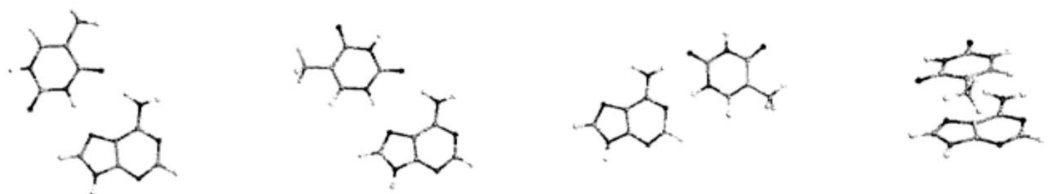

$\mathrm{p}(6473) ;-12.9 /-12.7 ; 8.5 \%$

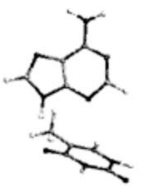

$\mathrm{p}(6271) ;-12.6 /-13.2 ; 4.4 \%$

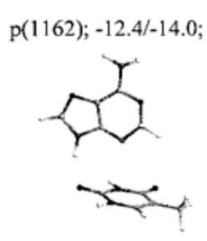

s1; $-11.4 /-10.8 ; 4.2 \%$

$\mathrm{t}(94) ;-11.4 /-9.1 ; 5.1 \%$

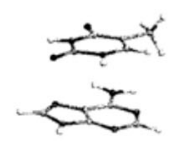

s2; $-11.1 /-10.5 ; 3.7 \%$

$\mathrm{t}(92) ;-10.8 / \mathrm{x} ; 1.9 \%$

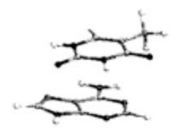

s3; $-10.5 /-9.0 ; 1.6 \%$

AT

FIG. 12

Structures of the adenine...thymine (non-methylated) base pairs. Numbers in parentheses refer to empirical and ab initio interaction energy and relative population (in $\mathrm{kcal} / \mathrm{mol}$ and \%). Reproduced from ref. ${ }^{146}$ with permission 
groups of adenine (and not through $\mathrm{N}^{6}$ amino group and ring $\mathrm{N}^{1}$ and $\mathrm{N}^{7}$ adenine positions like in the Watson-Crick and Hoogsteen structures). We are aware of the fact that these structures cannot occur in nucleic acids since the $\mathrm{N}^{9}$ position is blocked by the attached sugar ring. These results are, however, significant for gas-phase molecular beam experiments where the knowledge of the structure of the global minimum (or mostly populated minimum) is of key importance for interpretation of measured IR spectra. The Hoogsteen and Watson-Crick types of complexes represent the third and fourth local minima and are less stable by about $3 \mathrm{kcal} / \mathrm{mol}$ than the global minimum. The surprising energy preference of the global and the first two local energy minima was confirmed by correlated MP2 ab initio calculations using 6-31G** and 6-311G $(2 \mathrm{~d}, \mathrm{p})$ basis sets (structural types from MD/Q calculations were fully reoptimized at the ab initio level). For the sake of comparison with experiment performed at non-zero temperature, the relevant data are obtained from analysis of the Gibbs energy surface and not of the potential energy surface. The relative population of various structures (a quantity proportional to $\Delta \mathrm{G}$ of base pair formation) was determined by MD simulations in the NVE microcanonical ensemble. Although the stability order of the global and first two local minima is unaffected by including the entropy contribution, the stability order of the remaining structures is altered rather significantly in favour of stacked and T-shaped structures. The simulations further show that the population of the global minimum is about 35\%, meaning that experimental gas-phase studies are likely to detect a large number of mutually coexisting structures.

The potential energy and Gibbs energy surfaces of all ten canonical and methylated nucleic acid base pairs were studied ${ }^{148}$ and the results can be summarized as follows. More than a dozen of energy minima were located on the PES of each base pair. The global and first local minima of the non-methylated base pairs do systematically exhibit a planar $\mathrm{H}$-bonded structure, while T-shaped and stacked structures are less stable. Entropy does not play an important role and, therefore, the relative order of individual structures on the PES and FES does not differ to a large extent. However, methylation at purine $\mathrm{N}^{9}$ and pyrimidine $\mathrm{N}^{1}$ (positions where a sugar unit is attached) causes dramatic changes of the PESs and FESs. The main observation is that the most stable and most populated $\mathrm{H}$-bonded structures found for the non-methylated pairs are eliminated. For the methylated base pairs, entropy plays an important role and the structure of the global minimum does not usually correspond to the most populated structure. Frequently, it is a stacked structure which is the most populated one with entropy favouring stacking over $\mathrm{H}$-bonding. Calculations reveal that 
the PESs and FESs of most base pairs are very complex and are characterized by the coexistence of several structures, which makes assignment of various experimental characteristics difficult.

\subsection{Microhydrated Nucleic Acid Bases and Base Pairs}

It is well known that bulk water dramatically changes the structure, properties and reactivity of systems and this is particularly true for nucleic acid base pairs. Most of the base pairs in vacuo possess a planar $\mathrm{H}$-bonded structure but after placing them into water, the structure is changed to the stacked one. It is assumed that this is due to entropy of bulk water (hydrophobic effect). However, the question arises whether this is true and whether this pronounced effect is not just due to the action of few water molecules. Microhydration (addition of a few water molecules) has become popular among theoreticians as well as experimentalists and this process is under study in many laboratories. But even in microhydrated environment (in which temperature is very low), the static approach is no longer adequate and dynamic calculations are required. The dynamic structure of all ten possible nucleic acid and methylated nucleic acid base pairs hydrated by a small number of water molecules (from 1 to 16 ) was determined ${ }^{149}$ using molecular dynamics simulations in the NVE microcanonical ensemble with the Cornell et al. force field $d^{141}$. Figure 13 shows as an example the microhydration for adenine...thymine and 9-methyladenine...1-methylthymine base pairs. The presence of one water molecule does not affect the structure of any $\mathrm{H}$-bonded base pair but a higher number (mostly just two) of water molecules does. An equal population of the $\mathrm{H}$-bonded and stacked structures of adenine...adenine, adenine...guanine and adenine...thymine pairs is reached if as few as two water molecules are present, while obtaining equal population of these structures in the case of adenine...cytosine, cytosine...thymine, guanine...guanine and guanine...thymine pairs required the presence of four water molecules, and in the case of guanine...cytosine pair as many as six water molecules. A comparable population of $\mathrm{H}$-bonded and stacked structures for cytosine...cytosine and thymine...thymine base pairs was only obtained if at least eight water molecules hydrated the nucleobase dimer.

Methylation of bases changes the situation dramatically and the stacked structures were favoured over the $\mathrm{H}$-bonded ones even in the absence of water molecules in the majority of cases (this is, of course, also partially due to the fact that the hydrogen atom most suitable for the formation of $\mathrm{H}$-bonds was replaced by the methyl group). The data supply evidence that 

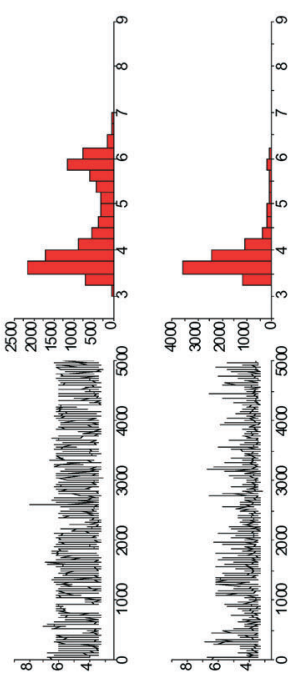

○

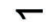

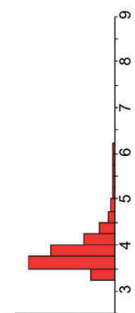
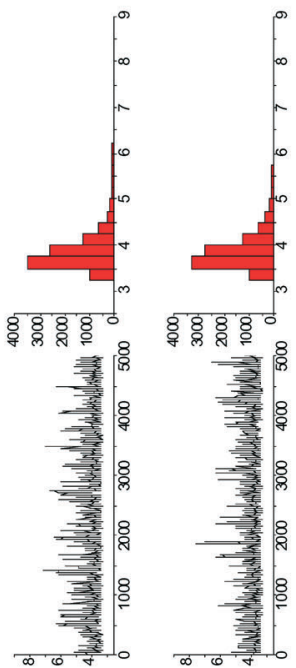

N

$\checkmark$
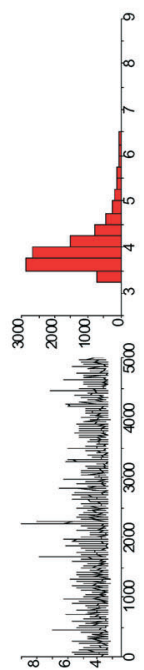

$\infty$

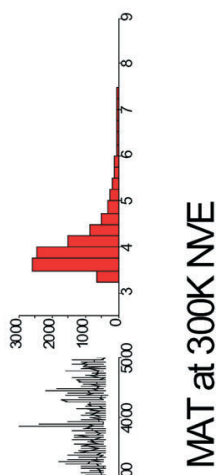

uo!̣ejndod
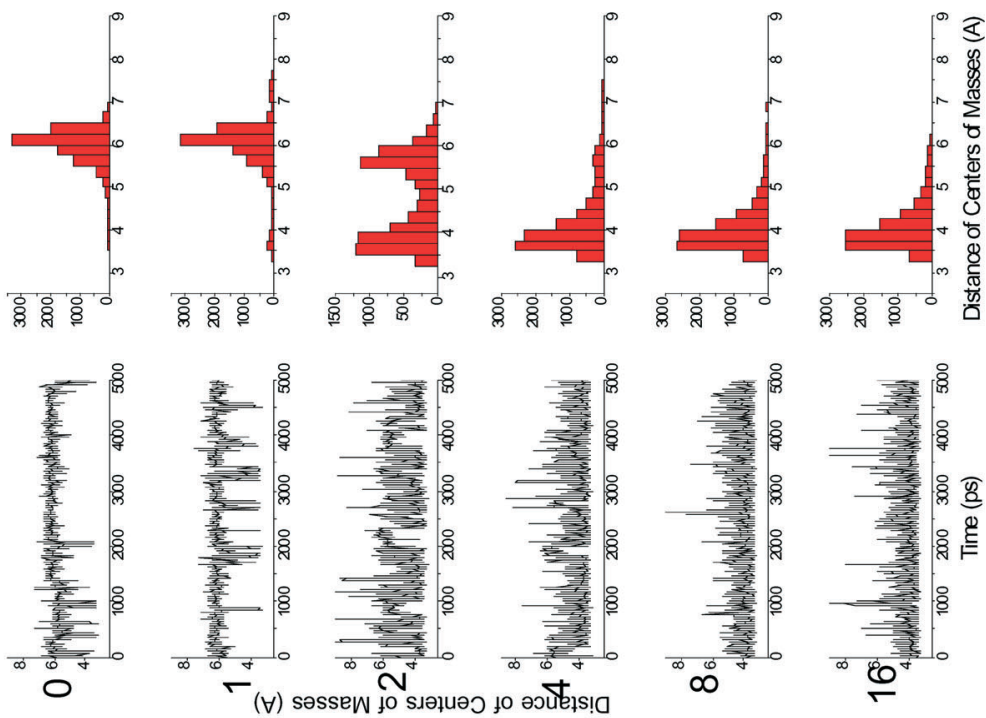

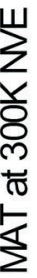

¿

든

है

4

के

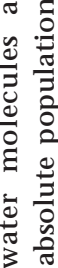

$6 \sum_{0}^{n}$

음

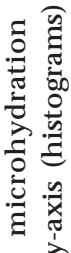

× ㅎํ ㅎํ

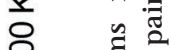

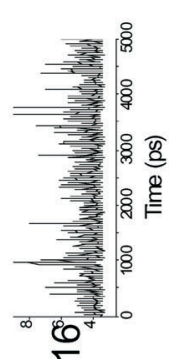

हิ ญ

ส

原

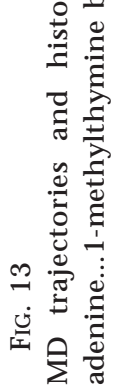

Collect. Czech. Chem. Commun. 2006, Vol. 71, No. 4, pp. 443-531 
the preferred stacked structure of DNA base pairs in a water solution might be due to the hydrophilic interaction of a small number of water molecules and not only due to hydrophobic effect of bulk water. The main conclusion from the study described, however, concerns the very pronounced role of microhydration which is able to dramatically change the structure and thus also properties of a cluster studied. Mono- and dihydration of the adenine...thymine pair was investigated recently ${ }^{149 b}$ using MD simulations and correlated ab initio optimizations. The latter study fully confirmed results from the previous study ${ }^{149}$ which were based on structures optimized with empirical potential.

Recently, the tautomeric equilibria of nucleic acid bases were studied in the gas phase, in a microhydrated environment and in aqueous solution ${ }^{150-153}$. It was shown that bulk water can change the relative stability of base tautomers significantly, for which the tautomerization of guanine serves as an excellent example ${ }^{151}$. The canonical form of guanine (the form which exists in DNA) and the 7-tautomer (hydrogen is placed at $\mathrm{N}^{7}$ instead of at $\mathrm{N}^{9}$ ) are the most stable forms in the gas phase, while the 7,9-tautomer (having hydrogens both at $\mathrm{N}^{7}$ and $\mathrm{N}^{9}$ ) is strongly destabilized (relative Gibbs energy amounts to about $20 \mathrm{kcal} / \mathrm{mol}$ ). The Gibbs energy of hydration of this tautomer is extremely high (about $-31 \mathrm{kcal} / \mathrm{mol}$ ) and as a result makes the relative Gibbs energy of this tautomer in an aqueous environment very favourable $(-11 \mathrm{kcal} / \mathrm{mol})$. A reason for this huge stabilization is a very large dipole moment of the 7,9-tautomer (9.1 D; canonical form has a dipole moment of $6.3 \mathrm{D}$ ). A very large dipole moment should be, however, also manifested by the large stabilization energy of this tautomer with water molecules; without water, the bare 7,9-tautomer is about $20 \mathrm{kcal} / \mathrm{mol}$ less stable than the canonical form. This very large energy difference is dramatically reduced upon complexation with one and two water molecules and becomes equal to about 13 and $9 \mathrm{kcal} / \mathrm{mol}$, respectively. A single water or two water molecules were not able to change the tautomeric equilibrium of isolated guanine and we expect that this is also true when a higher number of water molecules is added. Let us add that similar results were also obtained for microhydration of adenine and thymine. All these results indicate that microhydration plays a very significant role and must be properly considered.

\subsection{Carboxylic Acid Dimers}

Formic acid dimers ${ }^{154}$, acetic acid dimers ${ }^{155}$ and glycine dimers ${ }^{156}$ were studied in the same way as the above mentioned nucleic acid base pairs. 
The overall situation is similar in both cases and again, the global minimum of the PES sometimes differs from that of the FES. This is, in fact, a surprising result since the cyclic structures of the carboxylic acid dimers possessing two strong $\mathrm{C}=\mathrm{O} \ldots \mathrm{H}-\mathrm{O}$ hydrogen bonds (cf. Fig. 14) rank among the most stable complexes. The RI-MP2/TZVPP stabilization energies of formic acid and acetic acid dimers are 14.4 and $14.7 \mathrm{kcal} / \mathrm{mol}$, respectively, while the stabilization energy of the cyclic structure of the glycine dimer is slightly larger. The other structures of the first two acids are significantly less stable ${ }^{154,155}$. At low temperatures the cyclic structure of the formic acid dimer remains the global minimum. On increasing the temperature of the system, the population of the other cyclic structure (II on the left of Fig. 14) having one strong $\mathrm{C}=\mathrm{O} \ldots \mathrm{H}-\mathrm{O}$ and one weak $\mathrm{C}=\mathrm{O} \ldots \mathrm{H}-\mathrm{C}$ hydrogen bond becomes comparable; at higher temperatures (300 K) this structure even becomes the global minimum, as entropy considerations lead to stabilization
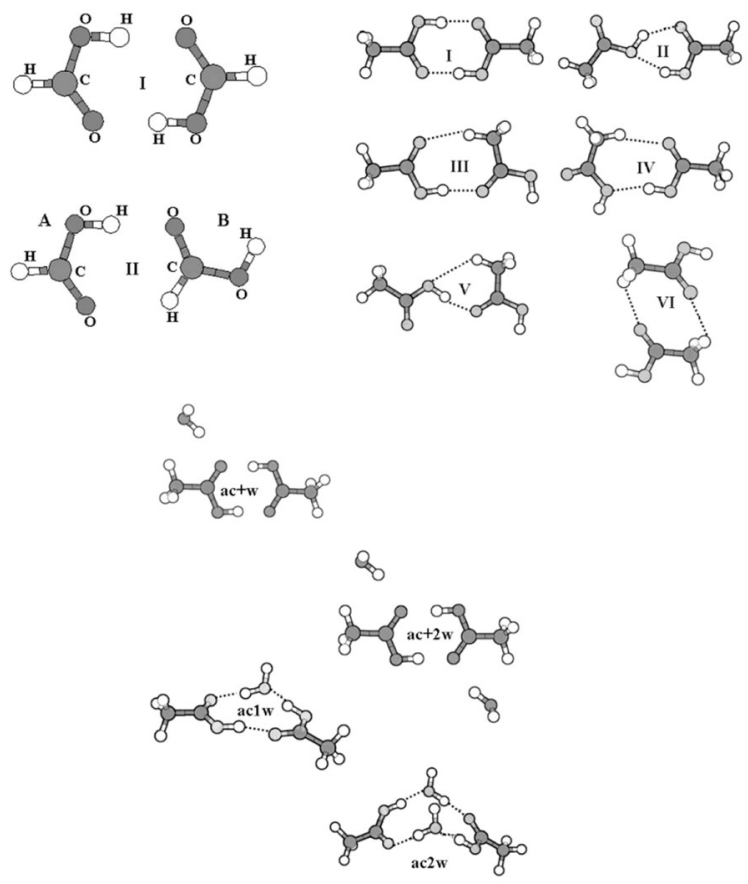

FIG. 14

Structures of carboxylic acid dimers and microhydrated carboxylic acid dimers. Reproduced from refs ${ }^{154,155}$ with permission 
of this structure. Evidently, entropy disfavours the strong (rather rigid) cyclic structure with two $\mathrm{C}=\mathrm{O} \ldots \mathrm{H}-\mathrm{O}$ hydrogen bonds and favours the weaker, more floppy cyclic structure having one strong $\mathrm{C}=\mathrm{O} \ldots \mathrm{H}-\mathrm{O}$ and one weak $\mathrm{C}=\mathrm{O} \ldots \mathrm{H}-\mathrm{C}$ hydrogen bond. This theoretically predicted behaviour was fully confirmed by the recent experiments of Sander et al. ${ }^{157}$ who measured IR spectra of the formic acid dimer in an argon matrix (though it must be mentioned that gas-phase experiments did not detect any evidence of this structure ${ }^{158}$ ). When performing their experiment at low temperature, the authors detected only the cyclic structure I (see Fig. 14) with two $\mathrm{C}=\mathrm{O} \ldots \mathrm{H}-\mathrm{O}$ hydrogen bonds. Upon increasing the temperature, the population of the second cyclic dimer II (see Fig. 14) having one strong $\mathrm{C}=\mathrm{O}$...H-O and one weak $\mathrm{C}=\mathrm{O} \ldots \mathrm{H}-\mathrm{C} \mathrm{H}$-bond becomes dominant. Surprisingly, experiments using helium droplets ${ }^{159}$ confirmed only the existence of the less stable cyclic structure. Since this cannot be due to entropy effects (the temperature in He-droplets is extremely low), further explanation of this phenomenon is required (cf. Section 5.5.)

In the case of acetic acid dimer the cyclic structure I, with two $\mathrm{C}=\mathrm{O} \ldots \mathrm{O}-\mathrm{H}$ bonds, remains the dominantly populated structure even when the temperature increases. Microhydration of the dimer (studied by MD/Q methods), however, shows changes in the dimer structure with the addition of only a single water moiety, resulting in breaking one of the $\mathrm{O}-\mathrm{H}$... O bonds and allowing the water molecule to be incorporated into the structure of the dimer (cf. Fig. 14). The second water molecule behaves in a similar way and usually breaks the other $\mathrm{O}-\mathrm{H}$... O bond, and similarly is also incorporated into the structure (cf. Fig. 14). On addition of further water molecules, different water-separated complexes of the acetic acid dimer are formed; the most frequently appearing complexes correspond to those incorporating one and two water molecules.

Experiments on the glycine dimer performed in Hedroplets ${ }^{159}$ gave unambiguous evidence of the presence of a free (unbound) $\mathrm{O}-\mathrm{H}$ group. Evidently, the dimer cannot have an expected cyclic structure with two $\mathrm{C}=\mathrm{O} \ldots \mathrm{H}-\mathrm{O} \mathrm{H}$-bonds ( $\mathrm{Cl}$ structure in Fig. 15) since the red shift of the $\mathrm{O}-\mathrm{H}$ stretching vibration in the dimer is large. The only way of interpreting the experiment is to admit that this cyclic structure is not present and another dimer structure having an unbound $\mathrm{O}-\mathrm{H}$ group is dominantly populated. We performed the MD/Q simulations ${ }^{156}$ using the Cornell et al. potential ${ }^{141}$ and found several hundreds of structures, of which 22 with the lowest energy were investigated in detail. To our surprise, the empirical potential favoured the stacked structure (S1 in Fig. 15) of the dimer over the cyclic C1 structure by about $3 \mathrm{kcal} / \mathrm{mol}$. Both $\mathrm{Cl}$ and $\mathrm{S} 1$ structures were re-optimized 
at ab initio correlated level (MP2/6-31G**) for which the results followed their expectations: The $\mathrm{C} 1$ structure was found to be the global minimum whilst the $\mathrm{S} 1$ structure was higher in energy by about $2 \mathrm{kcal} / \mathrm{mol}$. On the basis of our experience with stacking structures, however, we realized that the MP2/6-31G** description is not adequate (it is, on the other hand, quite sufficient for planar $\mathrm{H}$-bonding). Upon increasing the theoretical level (enlarging the $\mathrm{AO}$ basis set and increasing the level of electron correlation), we came to the conclusion that the stabilization energy of both structures is comparable, and from the extrapolation results obtained it may even be expected that the stacked structure becomes more stable. However, this surprising result did not solve the experimental finding since the stacked structure investigated also exhibits a rather large red shift of the $\mathrm{O}-\mathrm{H}$ stretching frequencies. Evidently, another explanation, as for the case of experiments on formic acid in He-droplets, is required (see Section 5.5.).

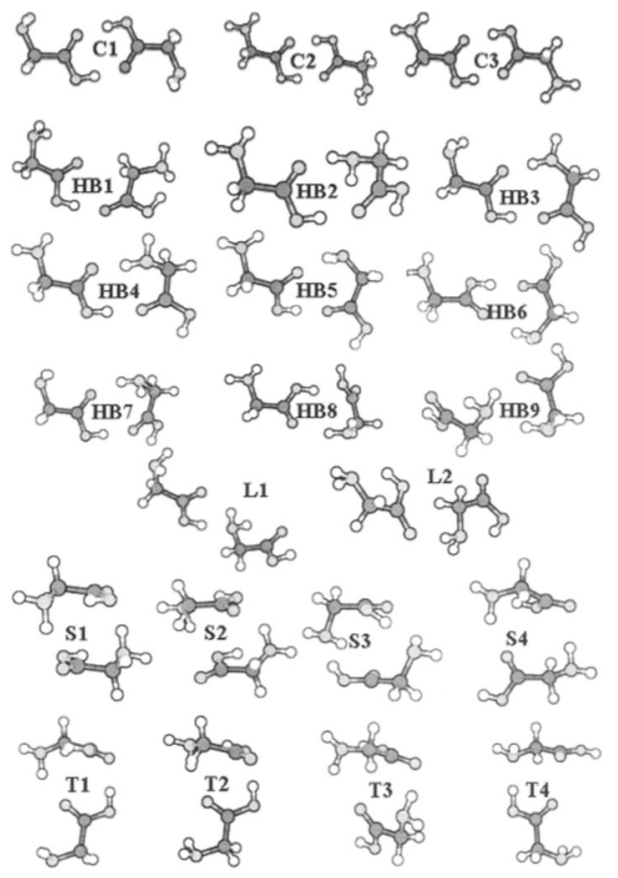

FIG. 15

Structures of glycine dimer. Reproduced from ref. ${ }^{156}$ with permission 


\subsection{Experimental Methods for Exploring Stationary Points on the PES: Stimulated Emission Pumping}

A problem associated with the spectroscopy of molecular clusters or molecules of biological interest in the gas phase is associated with the complexity of the potential energy surface. Complexity here means that the different conformers associated with stationary points on the potential energy surface, i.e. the global minimum and local minima with comparable energy, lead to several conformers populated in the supersonic jet expansion. This problem is related to the folding of polymers and proteins, which depends on the flexibility of sites whose energetic barriers are dictated largely by 'single bonds'. Molecules with flexible side chains can have several low-energy conformational minima with no significant energy barriers between them. From the experimental viewpoint a difficulty arises when distinguishing between these different conformers. If the energy barriers are sufficiently high and vibrational spectra are well resolved, then a distinction can be made by infrared hole-burning. By similar reasoning, this distinction may also be made by UV hole-burning, for which a resonant excitation into an electronically excited state, for instance the $S_{1}$ state, is used to deplete the population of one conformer (just replace the IR photon in Fig. 2 with a UV photon thus transferring population from the $\mathrm{S}_{0}$ into the $S_{1}$ ). However, for more complex molecules and clusters, these single photon hole-burning experiments become more and more difficult due to the inherent signal-to-noise limitations of signal depletion experiments. A recent experiment from the group of Zwier has shown that stimulated emission pumping-hole filling spectroscopy (SEP-HFS) can be used to determine different conformers in an elegant way by increasing their popula tion ${ }^{28 a}$. This was done for tryptamine, a molecule for which several conformers exist, which are stabilized by non-coval ent intramolecular interactions. The method is described in Fig. 16. Very close to the nozzle of supersonic jet expansion, i.e. in a region with high collison rates, a single conformer (e.g., conformer $A$ ) is selectively excited from its zero-point vibrational level to a certain vibrational level using $\operatorname{SEP}\left(\lambda_{1}\right.$ and $\left.\lambda_{2}\right)$. Once the barrier to isomerization is exceeded to form a given product (e.g., conformer $\mathrm{C}$ ), the isomerization followed by collisional cooling results in an increase in the population of the zero-point level of that conformer (C) downstream in the expansion. This change is detected, in this case, by laser induced fluorescence (LIF), using a third pulsed tunable UV laser $\left(\lambda_{3}\right)$. As $\lambda_{2}$ is tuned further towards higher energy, the ratelimiting barriers to other isomerization pathways are overcome, producing gains in the population in 


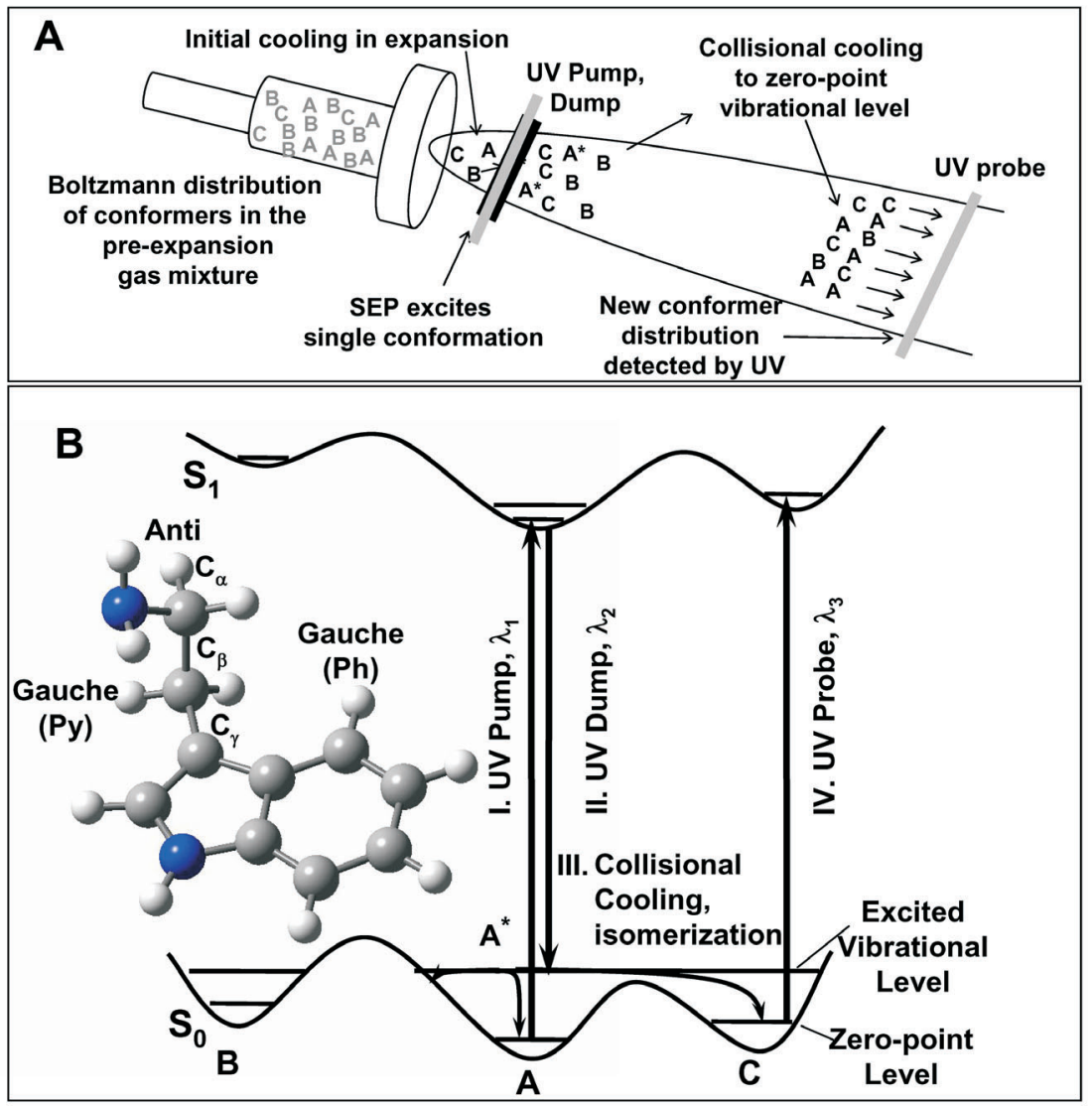

Fig. 16

(A) Schematic diagram of the spatial and temporal arrangement and (B) energy-level diagram for the SEP-HFS (stimulated emission pumping-hole filling spectroscopy) experiment. A single conformer (e.g., conformer A) is selectively excited to a certain vibrational level using SEP $\left(\lambda_{1}\right.$ and $\lambda_{2}$ ) from its zero-point vibrational level. Once the barrier to isomerization is exceeded to form a given product (e.g., conformer $\mathrm{C}$ ), the isomerization followed by collisional cooling will result in an increase in the population in the zero-point level of conformer $C$ downstream in the expansion. This change is detected via LIF using a third pulsed tunable UV laser $\left(\lambda_{3}\right)$. As the $\lambda_{2}$ wavelength is tuned further towards higher energy, the rate-limiting barriers to other isomerization pathways are overcome, producing gains in the population in other conformational zero-point levels (again, after collisional cooling). Reproduced from ref. ${ }^{28 a}$ with permission 
other conformational zero-point levels (again, after collisional cooling). Hence, this method can also be used to access conformers that are not at all populated in the supersonics expansion. Clearly, the method can also be used with the third pulsed tunable UV laser $\left(\lambda_{3}\right)$ ionizing the molecule for mass-selected REMPI detection. The experimental trick of SEP-HFS is to carry out the pump/dump stimulated emission early in the supersonic expansion, making it possible to recool the isomerized products into their vibrational zero-point levels from which they can be interrogated downstream.

\section{MOST IMPORTANT TYPES OF NON-COVALENT COMPLEXES}

\subsection{Classification of Non-Covalent Complexes}

Non-covalent complexes can be classified with respect to the dominant contribution to stabilization energy, by following a structural type, or simply on the basis of their size. Classification based on the former criterion is not unique since only very rarely one particular energy term is dominant. Probably the only complexes that fulfil this criterion are van der Waals complexes where the dominant attraction comes from the London dispersion energy; the only complexes that belong to this class are rare gas dimers. Electrostatic complexes can be considered as the other class, but here also the other energy contributions (induction and dispersion) contribute considerably. Further, these complexes mostly contain hydrogens and are called $\mathrm{H}$-bonded complexes (see Section 4.3.). The electrostatic term in $\mathrm{H}$-bonded complexes is mostly dominant but an important feature of these complexes is the non-negligible role played by induction and dispersion contributions. In addition, we meet difficulties with another energy contribution, namely the charge-transfer term. In the perturbation theory classification of this energy contribution is missing but it is included in the induction term. The concept of charge transfer is difficult since it is not experimentally detectable, but we know from experience that electron density is transferred from one system to another and this transfer takes place not only if one of the subsystems is a good electron donor and the other a good electron acceptor. An important feature of a $\mathrm{H}$-bonded complex is just this electron density transfer from the proton acceptor to the proton donor.

Classification of $\mathrm{H}$-bonds on the basis of structure is not unambiguous either and here we mostly recognize only the $\mathrm{H}$-bonded complexes which are planar, and stacked structures with vertical (i.e. vertical with respect to the main nodal plane of planar $\pi$-systems) $\pi-\pi$ interactions. Dominant attrac- 
tion with stacked structures is the London dispersion energy, but again the other energy terms are not negligible. The orientation of the subsystems in the stacked complex is due to the electrostatic term.

The specificity of $\mathrm{H}$-bonded systems does not come from the nature of non-covalent interaction (see Section 4.2.) but it is associated rather with their abundance in nature and their very specific and easily detectable spectroscopic manifestation. Besides $\mathrm{H}$-bonded complexes and other similar improper $\mathrm{H}$-bonded complexes, rather rare complexes with a dihydrogen bond will also be considered.

Classification based on the cluster size is also not unambiguous, but small complexes of different types exhibit some common specific features and it is thus advantageous to start a description of different non-covalent complexes with these systems. Let us only mention again that the benzene dimer with 24 atoms makes an arbitrary borderline between small (and medium) and extended non-covalent complexes.

\subsection{Small and Medium Size Non-Covalent Systems}

Many hundreds of non-covalent (vdW) complexes have been studied experimentally and theoretically in the gas phase of which we will cite a few dozens of studies for illustrative purposes. The systems will be divided into five groups: Rare gas atom complexes ${ }^{160}$, complexes between rare gas atoms and small and medium-size molecules ${ }^{161}$, complexes between small and medium-size species (mostly molecules) ${ }^{162}$, solvated (hydrated) ions ${ }^{163}$, and complexes between aromatic hydrocarbons and between those and other species $^{164}$.

It was only about ten years ago ${ }^{160 a}$ that the existence of the helium dimer was proven experimentally. The experimental binding energy of about $1 \mathrm{mK}$ ( $1 \mathrm{~K} \sim 4 \mathrm{cal} / \mathrm{mol}$ ) agrees with the result of numerous calculations of the potential energy well depth and interatomic distance based on both ab initio $^{160 b, 160 c}$ and empirical potentials ${ }^{160 d}$ (10.9 K and $2.97 \AA$ ). The first calculated value by Slater amounted to $8.9 \mathrm{~K}$ 160e; for a review, see ref. ${ }^{160 f}$ An explicitly correlated coupled cluster R12 calculation proves the high quality of the CCSD values ${ }^{160 \mathrm{~g}, 160 \mathrm{~h}}$. Ab initio potentials offer more accurate values than those based on the best measurements ${ }^{160 i, 160 j}$. Transmission of $\mathrm{He}$ and $(\mathrm{He})_{2}$ through nanoscale sieves lead to the determination of the size of $\left.(\mathrm{He})_{2}{ }^{160 k}\right)$. Experimental work on helium clusters was reviewed ${ }^{1601}$ and special attention was paid to $(\mathrm{He})_{2}$ and $(\mathrm{He})_{3}{ }^{160 m}$. In the course of time, interatomic potentials for various rare gas pairs have been improved (Ne...Ne ${ }^{160 n, 1600}, \mathrm{Ar} . . . \mathrm{Kr}^{160 p}$ ) with potential parameters for 21 homoge- 
neous and heterogeneous rare gas pairs currently available ${ }^{160 q}$. Complete basis set limits were obtained for high-level ab initio calculations for $(\mathrm{He})_{2}$, $(\mathrm{Ne})_{2}$, and $(\mathrm{Ar})_{2}{ }^{160 r}$. A critical survey of DFT studies on rare gas dimers ${ }^{160 \mathrm{~s}}$ also deserves attention.

An efficient procedure was developed which permits rapid estimates of the correlation energy in complexes of the RgX type $(\mathrm{Rg}=\mathrm{Ar}, \mathrm{Kr} ; \mathrm{X}=\mathrm{F}, \mathrm{Cl}$, $\mathrm{Br})^{161 a}$. Laser spectroscopy of GeAr and discussion on related systems is available in the literature ${ }^{161 b}$. $\operatorname{CCSD}(T)$ calculations were carried out for complexes of $\mathrm{Rg}$ atoms (He through $\mathrm{Xe}$ ) with the sulfur atom ground state $\left({ }^{3} \mathrm{P}\right)^{161 \mathrm{c}}$. A sophisticated potential energy surface has been calculated for the He.... $\mathrm{H}_{2}$ system ${ }^{161 d}$ and for $\mathrm{Rg} . . \mathrm{Br}_{2}(\mathrm{Rg}=\mathrm{He}, \mathrm{Ne}, \mathrm{Ar})$ complexes $^{161 e}$. Studies in liquid helium nanodroplets are very promising; this was suitably demonstrated for $\mathrm{Ar}_{\mathrm{n}} \ldots \mathrm{HF}$ complexes ${ }^{161 f}$. Accurate intermolecular potentials are available for the Rg...molecule (e.g., Ar...HCl) ${ }^{161 g}$ and Rg...ion complexes (e.g., Rg....CO ${ }^{+161 h}$. KrO- photoelectron spectra have been successfully interpreted by means of nonempirical calculations, and negative ion photoelectron spectra ( $\mathrm{Ar}, \mathrm{Kr}, \mathrm{Xe}$, and $\mathrm{N}_{2}$ with $\mathrm{O}^{-}$) have been recorded and interpreted ${ }^{161 j}$. Three-body effects have been analyzed in the $\mathrm{Ar}_{2} \mathrm{O}^{-}$trimer; the total three-body effect is given by the induction nonadditivity ${ }^{161 k}$. Attractive possibilities which offer photodissociation of non-covalent complexes have been well illustrated by analysis of the $\mathrm{CH}_{4} \ldots$ Ar system ${ }^{1611}$. Investigations of non-covalent complexes consisting of two or three subsystems (small- and medium-size species) provide a better understanding of these complexes and also allow for a deeper insight into behaviour of subsystems in biologically active supramolecules.

Specifically, the role of tunnelling in biology is still not sufficiently recognized. This process continues to attract attention, for instance for the vibrational dependence of tunnelling in $(\mathrm{HF})_{2}{ }^{162 a}$. The BSSE significantly influences the force constants and harmonic vibrational frequencies ${ }^{162 b}$ in $(\mathrm{HF})_{2}$ and $\left(\mathrm{H}_{2} \mathrm{O}\right)_{2}$; this problem was solved by using a procedure based on the 'chemical Hamiltonian approach' 162c.

Currently, however, high-quality results can be obtained by extrapolations to the CBS limit. Good- or high-quality calculations of parts of potential energy surfaces are available for $\mathrm{He} . . . \mathrm{ICl}$ and $\mathrm{Ne} . . . \mathrm{ICl}{ }^{162 d}, \mathrm{H}_{2} \mathrm{O} \ldots \mathrm{H}_{2}{ }^{162 e}$, and $\mathrm{N}_{2} \ldots \mathrm{HArF}$ and $\mathrm{N}_{2} \ldots \mathrm{HF}^{162 f}$ complexes. The $\mathrm{N}_{2} \ldots \mathrm{HArF}$ species exhibits a large blue shift of Ar-H stretching vibration frequency of $195 \mathrm{~cm}^{-1}$. The matrix isolation study allows detection of rotation around the $\mathrm{O}-\mathrm{O}$ axis in the $\mathrm{H}_{2} \mathrm{O}-\mathrm{OH}^{\cdot}$ complex ${ }^{162 \mathrm{~g}}$. Two isomers were studied in the $\mathrm{H}_{2} \mathrm{O}$-methanol complex by the diffusion Monte Carlo method with constraint dynamics; 
to obtain correct results the $\mathrm{CH}_{3}$ - and $\mathrm{OH}$-rotation must not be frozen ${ }^{162 \mathrm{~h}}$. A spectroscopic and theoretical analysis of the dimethyl ether.... $\mathrm{CO}_{2} \mathrm{com}$ plex ${ }^{162 i}$ lead to the structure $\left(\mathrm{CH}_{3}\right)_{2} \mathrm{O} \ldots \mathrm{CO}_{2}$. Morokuma's version ${ }^{162 \mathrm{j}}$ of the QM/MM procedure was used for establishing the structure of serin- $\mathrm{H}_{2} \mathrm{O}$ complexes ${ }^{162 k}$; in this connection the role of the hydrogen bonding on the conformers of valine ${ }^{162 l}$ should also be mentioned. Portions of the potential energy surfaces, three-body effects, and matrix studies (in some instances) were carried out for complexes consisting of three molecules or two molecules and one ion: $(\mathrm{Ar}-\mathrm{H}-\mathrm{Ar})^{+162 \mathrm{~m}}, \mathrm{Ar}_{2} \mathrm{HBr}{ }^{162 \mathrm{n}}, \mathrm{HXeOH}-\mathrm{H}_{2} \mathrm{O}^{1620}$, $\mathrm{I}^{-} \ldots\left(\mathrm{CH}_{3} \mathrm{CN}\right)_{2}{ }^{162 \mathrm{p}},(\mathrm{OCS})_{3}{ }^{162 \mathrm{r}}, \mathrm{CH}_{4}\left(\mathrm{H}_{2} \mathrm{O}\right)_{2}$ and $\mathrm{CH}_{4}\left(\mathrm{H}_{5} \mathrm{O}_{2}\right)^{+162 \mathrm{~s}}$, and $\left(\mathrm{H}_{2} \mathrm{O}\right)_{3}{ }^{-162 \mathrm{t}}$. Complete basis set characteristics were obtained for the $\mathrm{H}_{3}{ }^{+}$cation 'solvated' by one through four $\mathrm{H}_{2}$ molecules ${ }^{162 u}$.

Progress has been achieved in the calculation of hydration enthalpies of monoatomic cations $s^{163}$. Besides the long- and short-range interactions between the ion and water ligand, field stabilization was also taken into consideration. Calculated hydration enthalpies for 48 cations (alkali metal, alkaline earth metal, and transition metal ions with valency ranging from one to four) confirm experimental values.

Studies of interactions between planar aromatic hydrocarbons might be considered as purely 'academic' research. In fact the very opposite is true. Namely, this type of interaction plays a fundamental role in molecular recognition between supramolecular biosystems. The benzene dimer represents a valuable model system. Experimental studies (molecular beam electric resonance ${ }^{164 a, 164 b}$, rotational spectrum ${ }^{164 c}$ ) provided evidence for the T-shape dimer. Sophisticated computational studies ${ }^{164 d-164 h}$ lead to three stationary points on the $\left(\mathrm{C}_{6} \mathrm{H}_{6}\right)_{2}$ potential energy surface: T-shaped, parallel-displaced, and sandwiched, with binding energies of between 2 and $3 \mathrm{kcal} / \mathrm{mol}$. Analogous studies on the benzene-naphthalene ${ }^{164 i}$, naphthalene-naphthalene and naphthalene-anthracene complexes ${ }^{164 j}$ were performed, and an attempt was made to compensate the MP2 overestimation of the electron correlation with aromatic clusters by using only a mediumsize basis set.

The energetics, nuclear dynamics, and spectral features for the anthracene...He $(n=1,2)$ complexes were studied with combined quantum mechanical and empirical potential procedures ${ }^{164 k}$. Structure and spectra for complexes between substituted benzenes and various small molecules were studied: $\mathrm{H}_{2}{ }^{164 l}, \mathrm{H}_{2} \mathrm{O}$ and $\mathrm{H}_{2} \mathrm{~S}^{164 m}$, aniline... $\mathrm{N}_{2}{ }^{164 n}$, p-difluorobenzene... $\mathrm{N}_{2}{ }^{1640}$, and anisole $\left(\mathrm{H}_{2} \mathrm{O}\right)_{n}(\mathrm{n}=1,2,3)^{164 p}$. On the basis of an MD study ${ }^{164 q}$, the Gibbs energy of competition between the stacked and T-shaped structures was established for aromatic amino acids (phenyl- 
alanine, tyrosine). The interactions of benzene and fullerene with paramagnetic atoms, N, P, O, and S were analyzed, including evaluation of structures and binding energies by the MP2 theory ${ }^{164 r}$. Two interesting works on benzene-anion $\left(\mathrm{O}_{2}^{-}, \mathrm{NO}^{-}\right)$complexes have appeared in the literature ${ }^{164 s, 164 t}$. Finally, evidence has been accumulated that van der Waals complexes play an important role in electrophilic substitution of arenes; the same seems to hold true in some instances of bioreactivity.

\subsection{Hydrogen Bonding and Improper Hydrogen Bonding}

Hydrogen bonding is a subject which occupies quite a unique position in the realm of chemistry and it is linked to a story which started early in the 20th century. This is not an appropriate place to attempt a synthesis of all our knowledge; it seems, however, appropriate to try to pick up a few recent research tendencies. Moreover, it is virtually impossible to give a full account of hydrogen bonding. This is indicated, for instance, by Gálvez et al. ${ }^{165}$, who found that the keyword hydrogen bond, just in the period 1996-1999, is associated with about 16000 works in the Chemical Abstracts Database.

The hydrogen bond ( $\mathrm{H}$-bond) is one of the strongest and the most common type of non-covalent bond. It is difficult to define $\mathrm{H}$-bonds in terms of all the features ascribed to them in different branches of science; moreover, some older definitions now appear ill-conceived. The most recent and general definition states that the $\mathrm{H}$-bond describes an attractive interaction between two species (atoms, groups, molecules) in a structural arrangement where a hydrogen atom, covalently bound to a sufficiently electronegative atom of one species, is non-covalently bound to a place with an excess of electrons of the other species.

The $\mathrm{H}$-bond plays a key role in chemistry, physics, and biology and its consequences are enormous. Hydrogen bonds are responsible for the structure and properties of water, an essential compound for life. Further, $\mathrm{H}$-bonds also play a key role in determining the shapes, properties and functions of biomolecules. For a survey, the reader is referred to recently published monographs on H-bonding: 'The Weak Hydrogen Bond' 166 by Desiraju and Steiner, 'Hydrogen Bonding' 167 by Scheiner and 'An Introduction to Hydrogen Bonding' 168 by Jeffrey. The term 'hydrogen bond' was probably first used by Pauling in his paper on the nature of chemical bond ${ }^{8}$.

As mentioned above, the $\mathrm{H}$-bond is a non-covalent bond between electrondeficient hydrogen and a region of high electron density. Most frequently, 
an $\mathrm{H}$-bond is of the $\mathrm{X}-\mathrm{H}$... $\mathrm{Y}$ type, where $\mathrm{X}$ is the electronegative element and $Y$ is the contact with excess of electrons. $H$-bonds with $X, Y=F, O$ and $\mathrm{N}$ are the most frequent and most extensively studied ${ }^{166-168}$, though $\mathrm{X}-\mathrm{H} \ldots \pi$ hydrogen bonds (for $\mathrm{X}=\mathrm{O}$ and $\mathrm{C}$ ) have also been observed ${ }^{169-176}$.

Despite an enormous amount of literature dedicated to this subject an important question can still be raised: does an $\mathrm{H}$-bond represent some special type of non-covalent interaction? The answer is unambiguous - no. Any type of $\mathrm{H}$-bonding is stabilized by the same energy components as any other non-covalent bonding. The most important electrostatic contribution is accompanied by induction and dispersion contributions; these attractive terms are balanced by exchangerepulsion. There is nothing special about this energy decomposition and, from the point of view of intermolecular non-covalent bonding, $\mathrm{H}$-bonds do not form a special class. However, the $\mathrm{H}$-bond comes with a peculiar directionality: it is the sharing of the hydrogen atom between two electronegative atoms (the most frequent example) that causes the typical pseudo-linearity of the $\mathrm{X}-\mathrm{H}$...Y arrangement. Furthermore, sharing of a very light hydrogen atom between two electronegative atoms results in a rather dramatic change of properties of the $\mathrm{X}-\mathrm{H}$ covalent bond. This bond becomes weaker upon formation of the $\mathrm{H}$-bond and this weakening is the key factor in our understanding of the novel properties of the $\mathrm{X}-\mathrm{H}$ stretching vibrational frequency.

We can summarize that the characteristic features ${ }^{168}$ of the $\mathrm{X}-\mathrm{H}$...Y hydrogen bond are: (i) the $\mathrm{X}-\mathrm{H}$ covalent bond stretches in correlation with the strength of the $\mathrm{H}$-bond; (ii) a small amount of electron density (0.01-0.03 e) is transferred from the proton acceptor $(Y)$ to the proton donor molecule $(\mathrm{X}-\mathrm{H})$; (iii) the band that corresponds to the $\mathrm{X}-\mathrm{H}$ stretching shifts to lower frequency (red shift), increases in intensity and broadens. A shift to lower frequencies is called a red shift, and is the most important, easily detectable manifestation of the formation of the $\mathrm{H}$-bond. Indeed, it is the experimental basis for detection of $\mathrm{H}$-bonding. A red shift represents a 'fingerprint' of the $\mathrm{H}$-bonding and a 'no red shift - no $\mathrm{H}$-bonding' relation was until very recently a statement of dogma. None of the three books ${ }^{166-168}$ mentioned above on $\mathrm{H}$-bonding - which appeared at the very end of the last century - presents a single piece of evidence against this 'rule'.

However, reading the literature carefully, we found a few studies ${ }^{177-179}$ showing that the $\mathrm{X}-\mathrm{H}$...Y arrangement (which is attractive) can be also accompanied by an opposite geometrical and spectral manifestation. Instead of elongation of the $\mathrm{X}-\mathrm{H}$ bond accompanied by a red shift of the $\mathrm{X}-\mathrm{H}$ stretching vibration, the contraction of this bond and an associated blue 
shift of the respective stretching have also been detected. Moreover, the intensity of $\mathrm{X}-\mathrm{H}$ stretching vibrational frequency decreased upon formation of the $\mathrm{X}-\mathrm{H}$.... $\mathrm{Y}$ contact in strong contrast to 'standard' $\mathrm{H}$-bonding. Because several features of this novel bonding differ from standard $\mathrm{H}$-bonding whilst others are similar, we called it improper, blue-shifting $\mathrm{H}$-bonding ${ }^{180}$.

Before discussing the nature of improper, blue-shifting $\mathrm{H}$-bonding we must elucidate the origin of the 'standard' $\mathrm{H}$-bonding. There are two models describing the origin of $\mathrm{H}$-bonding: electrostatic and hyperconjugative charge-transfer (CT). The former model explains the formation of the $\mathrm{H}$-bond on the grounds of energetics: elongation of the $\mathrm{X}-\mathrm{H}$ bond increases the dipole moment of the proton donor and thus also the dipole-dipole attraction between proton donor and proton acceptor. Consequently, the total stabilization energy becomes larger. This model explains the geometrical, energetical and also vibrational characteristics of an $\mathrm{H}$-bonded complex. Is it thus necessary to include the concept of charge-transfer? Let us mention that the phenomenon of charge-transfer in hydrogen bonding is rather vague. We cannot detect it directly and its theoretical justification is not unambiguous. The first clear evidence supporting the CT concept comes from Coulson ${ }^{181}$ who showed that without allowing for electron transfer between proton acceptor and proton donor, one cannot explain the dramatic intensity increase in the $\mathrm{X}-\mathrm{H}$ stretching vibration upon formation of the $\mathrm{H}$-bond. Later, the concept of CT was proved by using the Natural Bond Orbital (NBO) analysis. Reed, Curtiss and Weinhold ${ }^{182}$ performed an NBO analysis for several typical H-bonded systems, demonstrating charge transfer from the lone pairs of the proton acceptors to the $\mathrm{X}-\mathrm{H} \sigma^{*}$ antibonding orbital of the proton donors. An increase in electron density in the antibonding orbitals results in a weakening of the $\mathrm{X}-\mathrm{H}$ covalent bond and this is accompanied by a concomitant lowering of the $\mathrm{X}-\mathrm{H}$ stretching frequency. The NBO analysis is thus a very useful technique for the study of the underlying principles of $\mathrm{H}$-bonding. It becomes clear at this stage that a realistic picture of the $\mathrm{H}$-bonding is based on a combination of both models; electrostatic and charge-transfer approaches complement each other, and only in borderline cases does one approach become dominant.

Are these models applicable also to the improper H-bond? Electrostatic models give, in many cases, qualitatively correct answers and can be used for a description of improper $\mathrm{H}$-bonds. Necessary condition for the successful use of this model is the negative sign of the proton donor dipole moment derivatives with respect to the stretching coordinate ${ }^{183}$; this means that the dipole moment increases with contraction of the $\mathrm{X}-\mathrm{H}$ bond. Such 
behaviour is not typical since in most cases the contraction of a bond is associated with a decrease in dipole moment, but for some classes of systems (e.g. $\mathrm{CHX}_{3}$, where $\mathrm{X}$ is halogen), this condition can be fulfilled. But improper $\mathrm{H}$-bond has also been detected in many complexes where the proton donor did not exhibit a negative gradient and in these cases the electrostatic model fails completely. So what is this telling us about the nature of the improper $\mathrm{H}$-bond? First of all, the overall charge-tran sfer between proton acceptor and proton donor is smaller than in the case of the $\mathrm{H}$-bonding; in this instance, the charge-transfer is mostly directed to the $\mathrm{X}-\mathrm{H} \sigma^{*}$ antibonding orbitals. In improper $\mathrm{H}$-bonding only a small fraction of charge-transfer goes to this orbital while largest portion of CT finally lands in the remote part of the proton donor. (On the basis of this difference the so-called $\mathrm{H}$-index ${ }^{184}$ was defined which unambiguously discriminates between $\mathrm{H}$-bonding and the improper $\mathrm{H}$-bonding.) However, even $\mathrm{a}$ small increase in electron density in the $\mathrm{X}-\mathrm{H} \sigma^{*}$ antibonding orbitals of the proton donor leads to significant weakening and elongation of this bond and not to the observed contraction. Contraction of $\mathrm{X}-\mathrm{H}$ bond would require a decrease in electron density in this antibonding orbital which seems to be impractical because the antibonding orbital should be the electrondonating and not electron-accepting orbital.

This unusual effect (a decrease in electron density in the $\sigma^{*}$ antibonding orbitals) was found ${ }^{185}$ in isomers of the guanine dimer (K9K9-1, K9K7-1 and $\mathrm{K} 7 \mathrm{~K} 7-1$, where $\mathrm{K} 9$ indicates the canonical tautomer and $\mathrm{K} 7$ indicates the tautomer having the hydrogen at N7 instead at N9; cf. Fig. 17) possessing two $\mathrm{N}-\mathrm{H} \ldots \mathrm{O}=\mathrm{C} \mathrm{H}$-bonds. Dimers were optimized at the HF/6-31G** level and vibrational frequencies were calculated. Amino groups in these isomers were not directly involved in the $\mathrm{H}$-bonding. Besides the red shifts of the $\mathrm{N}^{1}-\mathrm{H}$ stretching vibrations (supporting the $\mathrm{H}$-bonding character of these contacts), an unexpected blue shift of amino $\mathrm{N}-\mathrm{H}$ stretching vibrations was found, which fully agrees with published experimental results ${ }^{186}$. The blue shift of the amino group $\mathrm{N}-\mathrm{H}$ stretching vibrations in all the guanine dimer structures was clarified by the planarization of the guanine amino group (in the isolated guanine the amino group is strongly nonplanar). Absolute values of harmonic amino $\mathrm{N}-\mathrm{H}$ stretching frequencies and their shifts upon planarization were verified by performing two-dimensional anharmonic vibrational analyses. The planarization of the guanine amino group cannot be interpreted on the basis of an electrostatic model and is due to redistribution of electron density in subsystems upon dimerization; this redistribution occurred within the aromatic ring as well as at the amino group nitrogen and leads to the formation of a new mesomeric structure. The 
electron density decrease in the lone electron pair of amino nitrogen yields rehybridization of the respective atomic orbitals (changing them from the $\mathrm{sp}^{3}$ to the $\mathrm{sp}^{2}$ state) resulting in planarization of the amino group. The other consequence is decrease of electron density in the amino $\mathrm{N}-\mathrm{H} \sigma^{*}$ antibonding orbitals. The (amino) $\mathrm{N}-\mathrm{H} . . . \mathrm{O}=\mathrm{C}$ contacts can be thus described as improper, blue-shifting $\mathrm{H}$-bonds. An increased amino $\mathrm{N}-\mathrm{H}$ stretching frequency is the fingerprint of the planarization of the guanine amino group and is the first spectroscopic manifestation of the fact that the amino group in nucleic acid bases is nonplanar. Blue shifts of the amino $\mathrm{N}-\mathrm{H}$ stretching frequencies occur only if the amino group is bifurcated (cf. Fig. 17). The guanine dimer is the first complex where one proton acceptor ( $\mathrm{C}=\mathrm{O}$ group) is simultaneously linked to two proton donors $\left(\mathrm{NH}\right.$ and $\mathrm{NH}_{2}$ ) by the $\mathrm{H}$-bond and the improper, blue-shifting $\mathrm{H}$-bond.

The mechanism of the improper $\mathrm{H}$-bonding was originally ${ }^{180}$ explained by the charge transfer to the remote part of the proton donor leading there to elongation of $X-Y$ (mostly C-halogen) bonds, which subsequently causes contraction of the $\mathrm{X}-\mathrm{H}$ bond. The two-step mechanism suggested manifests itself by red shifts of the $X-Y$ stretching frequencies and a blue shift of $a$ $\mathrm{X}-\mathrm{H}$ stretching frequency, and for the dimethyl ether...fluoroform complex was indeed observed experimentally ${ }^{187}$.

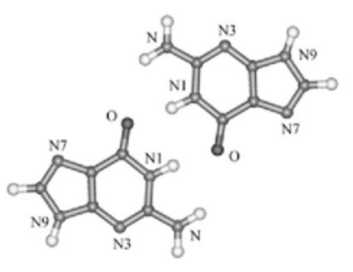

K9K9 - 1

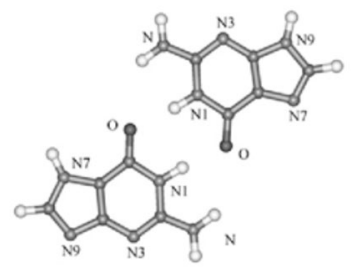

K9K7 - 1

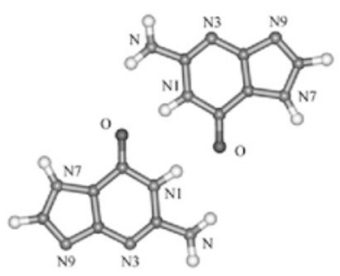

K7K7 - 1

FIG. 17

Structures of the guanine dimer. Reproduced from ref. ${ }^{185}$ with permission 
An elegant interpretation of the $\mathrm{H}$-bonding and the improper $\mathrm{H}$-bonding, based on application of Bent's rule, was recently presented by Alabugin and co-workers ${ }^{188}$. These authors described the role of the two effects present in both types of $\mathrm{H}$-bonded complexes: The hyperconjugative (charge-transfer) $\mathrm{X}-\mathrm{H}$ bond weakening and the rehybridization-promoted $\mathrm{X}-\mathrm{H}$ bond strengthening. The former effect, described above, is well recognized while the latter is not. The $\mathrm{X}-\mathrm{H}$ strengthening is due to an increase in the $\mathrm{s}$-character of the atom $\mathrm{X}$ hybrid orbital in the $\mathrm{X}-\mathrm{H}$ bond, which occurs upon a decrease in the $\mathrm{X}-\mathrm{H}$...Y distance. A direct consequence of Bent's rule is that a decrease in effective el ectronegativity of hydrogen (or an increase in hydrogen net charge) in an $\mathrm{X}-\mathrm{H}$ bond leads to an increase in the s-character of the atom $X$ hybrid orbital of this bond; the increase in $s$-character is associated with the bond contraction. Since the total s-character at atom $\mathrm{X}$ is conserved, an increase in s-character in the $\mathrm{X}-\mathrm{H}$ bond must be accompanied by a simultaneous increase in the $\mathrm{p}$-character (or a decrease in s-character) of other bonds connected to the $X$ atom; the increase in p-character is associated with the bond elongation. Both effects, i.e. the hyperconjugative (charge-transfer) $\mathrm{X}-\mathrm{H}$ weakening and the (rehybridization-promoted) $\mathrm{X}-\mathrm{H}$ bond strengthening are always present within any type of $\mathrm{H}$-bonded complex and the final picture (elongation or contraction) depends on the balance of these effects. The theory mentioned is consistent with structural reorganization in the remote part of the molecule suggested by us ${ }^{180}$ but does not require a two-step mechanism.

The direct consequence of the present theory is the fact that the blue shift decreases when going from $\mathrm{sp}^{3}$ - to $\mathrm{sp}^{2}$-hybridized atoms and completely vanishes with sp-hybridized atoms. This is, however, not completely true and even the first complex for which the improper $\mathrm{H}$-bonding was theoretically predicted, benzene dimer in the T-shaped structure ${ }^{189}$, belongs to this family. It is thus evident that still other mechanisms must exist. Let us repeat that the $\mathrm{C}-\mathrm{H}$ bond of the benzene proton donor contracts upon the formation of the T-shaped structure and the calculated blue shift is rather large. The most accurate anharmonic calculations ${ }^{189}$ show a shift of about $50 \mathrm{~cm}^{-1}$; presently, this shift has not been confirmed experimentally due to the fact that the spectral region where the shifted frequency is expected is overlapped by other stronger spectral bands. The shift is currently explained ${ }^{189-192}$ by the balance between dispersion attraction and exchange-repulsion and in the absence of other data, this explanation is still valid. The movement of the $\mathrm{X}-\mathrm{H}$ bond of the proton donor against a repulsion wall leads to contraction of the bond and a concomitant blue shift of its stretching frequency. This is the simplest and the most natural model 
for improper $\mathrm{H}$-bonding. Similarities and differences between $\mathrm{H}$-bonded and improper $\mathrm{H}$-bonded complexes were recently studied using the perturbation SAPT calculations. In the red-shifting complexes, the induction energy is mostly larger than the dispersion energy while, in the case of blue-shifting complexes, the situation is opposite. Dispersion as an attractive force increases the blue shift in the blue-shifting complexes as it compresses the $\mathrm{H}$-bond and, therefore, it increases the Pauli repulsion ${ }^{193}$.

Achievements in the real $\mathrm{m}$ of the $\mathrm{H}$-bonding were treated in a review ${ }^{194}$ dealing mainly with hydrogen bonds in the gas phase and solution. Hydrogen bonding in the solid state deserves special attention ${ }^{195}$. In this review thorough attention was paid to the history of the discovery of the hydrogen bond and to pioneering monographs. Features related to DFT calculations in the solid state are discussed with respect to periodicity in a paper dealing with ammonia and urea195b.

Recent papers of general importance deal with the decomposition of interaction energy in the $\mathrm{H}$-bonded dimers ${ }^{196 a}$, with the role of nuclear quantum effects on the structure of $\mathrm{H}$-bonded systems ${ }^{196 b}$, interpretation of the nature of the hydrogen bond in terms of topological descriptors (location of the bond critical point and geometry of the lone pair) ${ }^{196 c}$, and with charge transfer in $\mathrm{H}$-bonded clusters ${ }^{196 d}$.

The role that the $\mathrm{H}$-bonding plays in molecular recognition ${ }^{197 a, 197 b}$ and with spontaneous self-organization ${ }^{197 c, 197 d, ~ n o t ~ o n l y ~ w i t h ~ b i o l o g i c a l ~ s y s-~}$ tems, is overwhelming. Nitrogen-, oxygen-, and fluorine-containing compounds represent systems for classical $\mathrm{O}-\mathrm{H} \ldots \mathrm{O}, \mathrm{N}-\mathrm{H} \ldots \mathrm{O}$, and $\mathrm{N}-\mathrm{H} \ldots \mathrm{N}$ bonds; significantly weaker $\mathrm{C}-\mathrm{H} \ldots \mathrm{O}, \mathrm{C}-\mathrm{H} \ldots \pi$, and $\mathrm{N}-\mathrm{H} \ldots \pi$ have been investigated only in recent years. Valuable references on these types of interactions are available in a recent publication ${ }^{197 c}$.

Hydrogen bonding in water clusters is an evergreen ${ }^{198}$. A quarter of century elapsed before the pioneering work on $\mathrm{H}_{2} \mathrm{O}$ and $\mathrm{D}_{2} \mathrm{O}$ clusters in the gas phase $^{198 a}$ was complete. Water clusters were studied in hydrophobic solvents (liquid helium droplets, solid parahydrogen, and $\mathrm{CCl}_{4}$ ) and also quantum-chemically ${ }^{198 b}$. Three-body interactions in water clusters were studied, which led to a new ab initio three-body potential ${ }^{198 c, 198 d}$. New water chains and layers have been observed, including $\left(\mathrm{H}_{2} \mathrm{O}\right)_{12}$ rings $^{198 e}$. $\left(\mathrm{H}_{2} \mathrm{O}\right)_{20}$ clusters have attracted attention for years; there are 30026 symmetrydistinct ways of arranging twenty water molecules ${ }^{198 f}$. The presence of ions in water has only a very small effect on the $\mathrm{H}$-bond structure of liquid water ${ }^{1989}$. Increasing attention has been paid to the water structure in solid hosts ${ }^{198 h, 198 i}$. 
With regard to the role which the hydrogen-bonded oligomers of $\mathrm{HF}$ have played, a classic work on $(\mathrm{HF})_{2}, \mathrm{HFDF}$, and $(\mathrm{DF})_{2}$ should be quoted ${ }^{199 a}$ together with a work on $\mathrm{J}-\mathrm{J}$ correlations in state-to-state photodissociation ${ }^{199 b}$ of $(\mathrm{HF})_{2}$.

Within the framework of classic $\mathrm{H}$-bonds ${ }^{200}$, various complexes have been studied, e.g., formamide-methanol200a, oligomers of formamide and thioformamide $200 \mathrm{~b}$, indole-pyrrole $\mathrm{e}^{200 \mathrm{c}}$, together with the role of $\mathrm{H}$-bonding in Schiff bases ${ }^{200 d}$, furan-hydrogen halides (rotational spectroscopy) ${ }^{200 e}$, strong complexes (e.g., $\left.\mathrm{H}_{3} \mathrm{~N}-\mathrm{HF}\right)^{200 f}$, vibrational coupling through individual $\mathrm{H}$-bond chains ${ }^{200 \mathrm{~g}}$ in helices of pentapeptides), and a strong $\mathrm{Br}-\mathrm{H}-\mathrm{Br}^{-}$ bond $200 \mathrm{~h}$. It is possible to obtain evidence about the strength of the $\mathrm{H}$-bonding from NMR isotope shifts ${ }^{200 i}$.

It is now widely recognized and accepted that correction for BSSE in calculations of $\mathrm{H}$-bonded systems is highly desirable. The use of a function counterpoise procedure is reasonably efficient ${ }^{200 j}$, though BSSE corrections are unnecessary when we are able to get extrapolated $\Delta \mathrm{E}$ values for infinite basis sets ${ }^{200 k}$.

The existence of the $\mathrm{C}-\mathrm{H}$...O bond has been felt intuitively since the middle of the past century. In 1962 a paper appeared on the presence of this bond in crystals ${ }^{201 a}$. Since the turn of the last century, however, the lack of literature in this area has changed significantly and the number of works has been increasing very rapidly.

Most often theoretical and experimental tools are used simultaneously. On the theoretical side, the MP type of calculations and economically attractive DFT procedures with e.g. B3LYP functional are most frequently used. Infrared spectroscopy and NMR are the most powerful tools for characterizing hydrogen bonding ${ }^{201}$. In this illustrative set of systems, bonds such as $\mathrm{C}-\mathrm{H} \ldots \mathrm{O}, \mathrm{C}-\mathrm{H} \ldots \mathrm{N}, \mathrm{C}-\mathrm{H} \ldots \mathrm{F}, \mathrm{C}-\mathrm{H} \ldots \pi, \mathrm{O}-\mathrm{H} \ldots \pi$, are included. Also here extrapolations to an infinite basis set were carried out ${ }^{201 q}$, with regard to the formation of acetylene dimer $(\Delta \mathrm{E})$ and its isomerization $\left(\Delta \mathrm{E}^{\neq}\right)$to an equivalent form.

During the last few years the term 'halogen bonding' has been occurring in the literature again and again ${ }^{202}$. This term refers to an attractive interaction between a halogen atom with a free electron pair of atoms in the neighbourhood 202a. Evidence has been accumulated that close chalcogen-chalcogen contacts are responsible for the formation of tubular structures in solids ${ }^{202 b}$. The $X . . . X$ distance in numerous instances is significantly shorter than the sum of van der Waals radii of $X$; the interaction energy amounts to $0.5-2 \mathrm{kcal} / \mathrm{mol}$. A theoretical analysis of halogen-halogen interactions is available $e^{202 c}$, in which the authors define two types of inter- 
action. Papers on resonance-assisted intramolecular $\mathrm{X} \ldots \mathrm{X}(\mathrm{X}=$ halogen) interactions ${ }^{202 d}$, on polyhalomethane-halogen molecular complexes ${ }^{202 e}$, and on $\mathrm{N}$...Br bonding ${ }^{202 f}$ indicate the frequent occurrence of such bonding. No doubt, this type of interaction plays an important role not only in 'crystal engineering' but also in the formation of various types of supramolecular systems. However, we do not like the tendency to search for a close parallelism with hydrogen bonding. The hydrogen bond is really a bond sui generis; the other types of bonding mentioned are obviously due to dispersion (London) interactions.

What has been considered for years as 'nonbonded steric repulsion' between hydrogen atoms ( e.g., in planar biphenyl), contributes in fact to the stabilization of the system by about $10 \mathrm{kcal} / \mathrm{mol}{ }^{203 a}$. The authors stress that also another type of $\mathrm{H}-\mathrm{H}$ interaction exists, the interaction labelled 'dihydrogen bonding' (see the next paragraph), a bond with the hydride ion in the role of a base. Recently, the shortest $\mathrm{H}$...H distance $1.95 \AA$ was found by neutron diffraction in a derivative of D-ribofuranose ${ }^{203 b}$. For the sake of completeness, we need to add that a remarkably strong, very short $\mathrm{H}-\mathrm{H}$ bond $(0.095 \AA)$ exists in the radical cation of the methane dimer, which has the linear structure $203 \mathrm{C} \mathrm{H}_{3} \mathrm{C}-\mathrm{H}-\mathrm{H}-\mathrm{CH}_{3}{ }^{\cdot+}$.

\subsection{Dihydrogen Bonding}

Complexes with the dihydrogen bond are much less numerous than the previous ones but represent an interesting class of non-covalent complexes. The dihydrogen bond of the type $\mathrm{M}-\mathrm{H} \ldots \mathrm{H}-\mathrm{Y}$ was originally found ${ }^{204}$ in metal complexes ( $M=$ metal), but was also later detected ${ }^{205}$ in the $\mathrm{H}_{3} \mathrm{BNH}_{3}$ dimer. The unusually high boiling point of this system gave evidence for $\mathrm{a}$ strong intermolecular attraction that was finally shown to be of dihydrogen origin.

The explanation for this unconventional $\mathrm{H}$-bond is surprising but straightforward 206: two hydrogens may only interact if one is positive and the other negative. This can be realized if one hydrogen is bound to an electropositive element whilst the other is bound to a very electronegative element. The most common elements that are more electropositive than hydrogen are boron, alkali metals and heavy transition metals; dihydrogen bonding was found for various complexes containing these elements. The hydrogen bound to this element becomes negative whilst the one bound to a very electronegative element becomes positive. Thus, there is an electrostatic attraction between these hydrogens. Literature on this type of bond- 
ing is growing and many complexes with different types of dihydrogen bond are now known to exist ${ }^{207}$.

\subsection{Cooperative Hydrogen Bonding}

Dannenberg and co-workers carried out several DFT/B3YP calculations on peptide models that consider the effects of cooperative interactions with proximate $\mathrm{H}$-bonds and local geometry ${ }^{208}$. The calculations predict that cooperative interactions with other $\mathrm{H}$-bonds within a $\mathrm{H}$-bonding chain can significantly increase the strength of these coupling. Such increases are due to a combination of the presence of the neighbouring $\mathrm{H}$-bonds and the slight increase in the $\mathrm{C}=\mathrm{O}$ distances expected for peptide $\mathrm{H}$-bonds near the centres of $\mathrm{H}$-bonding chains. The results mentioned can be questioned since the BSSE was not considered.

\subsection{Nucleic Acid Components, Nucleic Acids, Peptides and Proteins}

The cooperative contribution to the $\mathrm{H}$-bond formation energy (i.e., formation of two $\mathrm{H}$-bonds) is similar in A...T and G...C base pairs. As the A...T interaction energy is smaller, the cooperative interaction (contributing to about $30 \%$ of its value) is larger by a factor of two than that with the G...C pair ${ }^{209}$. In connection with electron affinity calculations of the G...C base pair, it was shown (DFT) that the unpaired electron is essentially localized on the cytosine moiety ${ }^{210}$. Tautomeric mispairing and the Watson-Crick to Hoogsteen conversion were analyzed in the A...T pair ${ }^{211}$. In a study aimed at intercalation of carcinogens, a model interaction between benzo(a)pyrene or its derivatives with the G...C base pair were investigated $^{212}$ with convincing results on the structure of the complex formed. The influence of substituents (both electronegative and electropositive) on the hydrogen bond energy were studied (MP2) in the A...U, A...T and G...C Watson-Crick base pairs ${ }^{213}$. A new base-pairing motif (including imidazopyridopyrimidine nucleosides) can lead to forms having four hydrogen bonds ${ }^{214}$. The distance between the subunits in the uracil...adenine radical anion is significantly less than in the neutral parent system ${ }^{215}$. The restricted open-shell Kohn-Sham method was used successfully ${ }^{216}$ for geometry optimization of the six lowest energy tautomers in the first excited singlet state; while the enol tautomers remain nearly planar, the keto tautomers deviate significantly from planarity.

$\beta$-Hairpin peptides are used for probing various aspects of conformational stability. The energy stabilization due to Glu-Lys salt bridges was estab- 
lished by MD calculations using the Cornell et al. potential ${ }^{217 a}$. Adsorption of the first water molecule on unsolvated alanine-based peptides with adopted secondary structure was measured and enthalpy and entropy changes established. MD simulations satisfactorily reproduce experimental findings ${ }^{217 b}$. The positive role of water molecules in protein-protein interaction was proved; this concerns in particular stabilization of positively or negatively charged groups in protein interfaces ${ }^{218}$. Very recently, a step forward was made by a more realistic description of non-covalent interactions between protein molecules in an electrolyte solution ${ }^{219}$. The activation entropy is an essential component of the driving force of the gas-phase dissociation of proteins and protein-ligand complexes ${ }^{220}$. Extraordinarily tempting is a comparison of structure and other features of protein-ligand complexes in the gas and aqueous phases. Unfortunately, it is not clear as to what extent specific interactions in the gas phase are preserved. However, evidence has been accumulated, that one of the specific hydrogen bonds in a protein-oligosaccharide non-covalent complex remains untouched ${ }^{221}$. A model was developed to describe DNA-protein complexes including aspects of mechanical modelling, electrostatic interactions and hydrodynamics capable of describing inhomogeneous effects ${ }^{222}$. This model is an essential supplement to models describing DNA-protein complexes at the atomic level.

\section{INTERPRETATION OF EXPERIMENTAL RESULTS}

Interpretation of experimental data on non-covalent systems is not straightforward and requires a detailed knowledge of studied non-covalent systems; moreover, several additional factors should be taken into consideration. The first factor concerns the temperature of experiment. This is associated with the fact that in the world of non-covalent species entropy always plays an important, sometimes even decisive role. When translational, vibrational and rotational temperatures are low, we can safely use information obtained from the PES. When, however, the temperature is non-zero, then it is inevitable that the system will pass from the PES to the Gibbs energy surface (FES). The second factor is associated with the fact that the whole surface should be known, which means that all energy minima should be considered and must be properly weighted. The experimental information on, e.g., stabilization energy should be thus considered not only for the most stable or most highly populated structure but also other structures should be taken into account. Finally, the role of environment should be properly understood and adequate theoretical calculations 
should be performed. It is no longer considered appropriate to perform calculations in the gas phase and to use these results for interpretation of liquid-phase experiments, the fact which is now more or less accepted in the scientific community. We will show, however, some less frequent examples concerning the interpretation of experimental results from He-droplets experiments.

\subsection{Phenol...Argon and Benzene Dimer in vacuo}

In the following section we will demonstrate the problems in interpreting experimental data for two extensively studied non-covalent complexes, phenol...Ar and the benzene dimer. A very important general problem concerns the question 'will a non-covalently bound system assume a planar or abovering structure?'. This question is of particular importance concerning the structure and dynamics of nucleic acid base pairs (see Section 4.6.). We want to illustrate this with a very simple example, the phenol... argon complex. In contrast to benzene...argon, extensively studied by REMPI and other excited state (e.g., LIF) spectroscopy 223-227, large benzene clusters ${ }^{228}$ and benzene...A $\mathrm{Ar}_{\mathrm{n}}{ }^{229,230}$ clusters, complexes containing phenol are interesting because two principal ligand binding sites are available: via the $\mathrm{OH}$ group and via interaction with the aromatic $\pi$-system. The phenol...argon complex can be expected to show two main structural motifs: a plane hydrogen-bonded geometry with the argon bonded to the $\mathrm{OH}$ group and an above-ring van der Waals-bonded geometry with the argon bonded to the aromatic $\pi$-system.

We investigated the phenol...argon complex in the neutral ground state and the cation ground state by ab initio methods and also studied it experimentally using REMPI and ZEKE spectroscopy 231,232. The energetics obtained are shown in Fig. 18. Experimental vibrational frequencies for the $S_{1}$ state and the cationic ground state $\mathrm{D}_{0}$ and calculated frequencies for the $\mathrm{S}_{0}$ and the $D_{0}$ are summarized in Table I. For the neutral cluster, the abovering $\pi$-bound structure is the global minimum and the Ar...H-O-Ph hydrogen-bonded conformer does show up as a very shallow local minimum or, more likely, as a transition state, according to the vibrational frequency calculation at the MP2/cc-pVDZ level of theory (Table I). However, for the ionic state, the calculation confirms that the hydrogen-bonded conformer is indeed the global minimum and the above-ring $\pi$-bound structure is a local minimum. It should be noted that phenol...argon is, at the simplest level, comparable with the concept of going from hydrogen-bonded to stacked structures in the nucleic acid base pairs. 

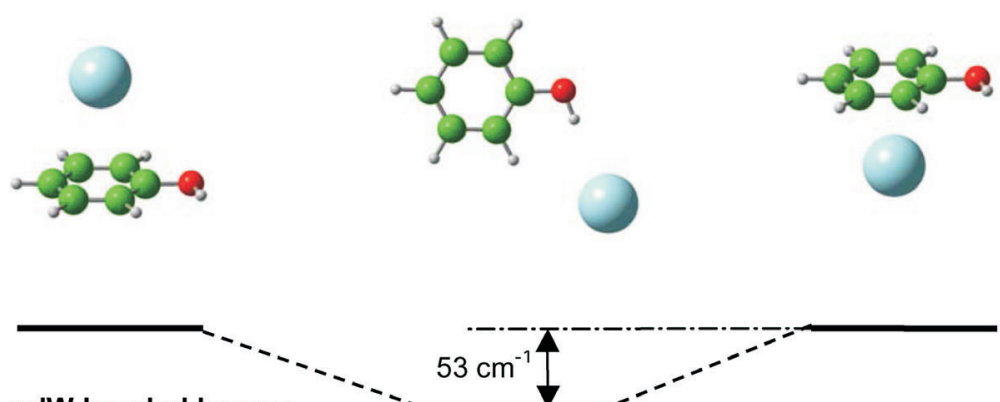

vdW bonded Isomer

\section{Hydrogen-bonded Isomer}

\section{Hydrogen-bonded TS}

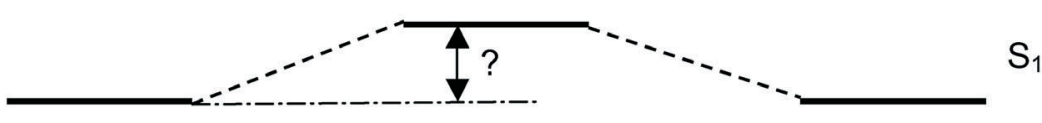

vdW bonded Isomer

\section{Hydrogen-bonded TS}

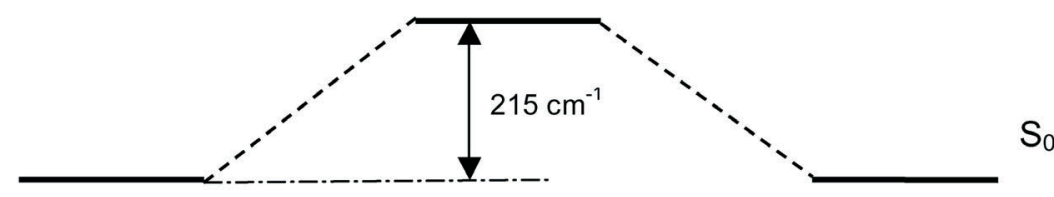

\section{vdW bonded Isomer}

\section{FIG. 18}

The energetics for the phenol...argon complex. For the $S_{0}$ neutral ground state, the above ring structure has a substantially higher stabilization energy than the hydrogen-bonded conformer, but the energy ordering is reversed in the cation. Reproduced from ref. ${ }^{231}$ 
The experimental study of the phenol...argon system showed very conclusively that for the $\mathrm{S}_{1} \leftarrow \mathrm{S}_{0}$ transition, as shown in Fig. 19, the above-ring structure is observed in the REMPI and MATI experiment ${ }^{232,55}$. This result was conclusively obtained from the simulation of the rotational band structure of the $\mathrm{S}_{1} \leftarrow \mathrm{S}_{0}$ transition $231,232,55$. For this procedure, which has been pioneered by Simons and co-workers for molecules of biological interest in

TABLE I

Intermolecular vibrational frequencies for phenol...Ar calculated at MP2 (aug-cc-pVDZ) level. From ref. 231

Intermolecular vibration, $\mathrm{cm}^{-1}$

$\mathrm{S}_{0}$

Neutral ground state

$\mathrm{D}_{0}$

Cationic ground state
vdW bonded isomer

hydrogen-bonded TS

vdW bonded isomer

hydrogen-bonded isomer

experimental value $b_{x}$

25

$-5$

5

18

14 $b_{y}$

40

48

$21 \quad 35$

37

50

61

66

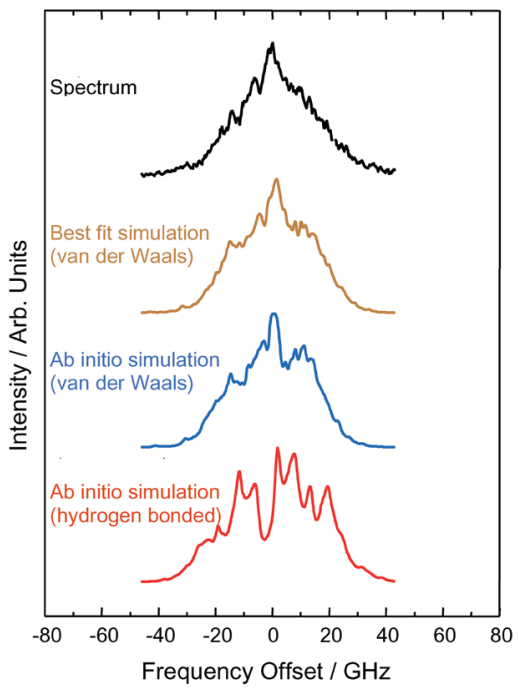

FIG. 19

Rotational contours for the phenol...argon $\mathrm{S}_{1} \leftarrow \mathrm{S}_{0}$ transition: (a) for the van der Waals above-ring structure, (b) for the hydrogen-bonded local minimum structure. Reproduced from ref. $^{231}$ (improved fitting compared to ref. ${ }^{232}$ ) 
the gas phase ${ }^{24,25}$ different conformers, such as in-plane or above-ring, do show spectral bands with a rather different rotational contour. The fitting procedure is carried out by simultaneously optimizing the direction of the transition moment and the two sets of rotational constants for the $S_{0}$ and the $S_{1}$. An improved result for this fitting procedure, compared with the one in ref. ${ }^{232}$, is shown in Fig. $19^{231}$. Even with only partial rotational resolution, the fitting procedure results in a very satisfactory accuracy for the rotational constants. For the cation, the ZEKE spectra obtained via several different intermediate vibrational states of phenol...argon showed very clearly that the cation structure observed in the experiment must also be the van der Waals above-ring structure ${ }^{55,232}$.

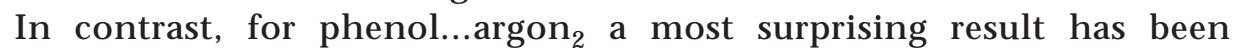
obtained recently: upon ionization one of the two argon atoms moves to the hydrogen-bonded $\operatorname{site}^{45}$. Upon cation formation, the barrier between the $\pi$-bound and the hydrogen-bonded geometry seems to be very low, resulting in a fast geometry change. The ZEKE/MATI spectra of phenol....argon 2 reveal this geometry change in great detail 233,234 .

\subsection{The Benzene Dimer: A Test Case for Our Understanding}

As a good test case for our understanding of the interplay of electrostatic and dispersion forces, we may consider the case of $\pi-\pi$ interactions in the benzene dimer.

Unlike H-bonded systems, where dipolar forces are dominant in deciding the ultimate geometry of the complex, the benzene dipole moment is vanishing for symmetry reasons. Nevertheless, the benzene dimer geometry is determined by electrostatic quadrupole-quadrupole interaction (the benzene quadrupole moment amounts to $8.5 \pm 1.4 \mathrm{D} \AA)^{235}$. Being ultimately weaker than dipole-dipole interactions, the balance of the electrostatic quadrupole-quadrupole interaction (which can be attractive or repulsive) and attractive dispersion interaction becomes much more important and, consequently, presents a difficult challenge to experimental and theoretical interpretation in terms of structure and energetics determination.

Indeed, in both experimental and computational studies published, various proposals for the expected global minimum structure on the benzene dimer PES exist. If, for a moment, we neglect existence of the benzene quadrupole, then we may only conclude that the dimer structure will most likely take a completely overlapping 'sandwich' structure (see Fig. 20) to allow for the maximum dispersive interaction between the two rings. Experiments performed about 15 years ago initially supported this conclu- 
sion $^{236,237}$, but were soon contradicted by hole-burning ${ }^{238}$ and microwave ${ }^{239}$ studies. Both these studies supported a T-shaped (see Fig. 20) global minimum structure and, in ref. ${ }^{237}$, showed evidence of three isomeric forms.

Consideration of the quadrupole-quadrupole interaction is important to account for the observation of all possible dimer species; this has been amply demonstrated by qualitative estimates ${ }^{240}$ of this contribution and, subsequently, by ab initio calculations ${ }^{164 e, 241}$. In the MP2 and CCSD(T) computational studies, a T-shaped structure was also found to be the global minimum in accord with experiment. Similar studies conducted at the same time showed that the Perdew-Wang functional describes the benzene dimer PES relatively correctly while the BLYP and B3LYP functionals fail 242 . The importance of the quadrupole moment for the stabilization of the benzene dimer was al ready shown in 1983 by Karlstrom et al. ${ }^{243}$

With respect to refs ${ }^{240,241}$, we may conclude that whilst a consideration of the quadrupole-quadrupole interaction is certainly important, the failure of the BLYP and B3LYP models - and the relatively good performance of correlated ab initio calculations - suggests that the dispersive contribution to binding is significant to the extent that both electrostatics and dispersion must be treated in an even-handed manner. Further computational studies have examined this requirement in detail. Whilst MP2/aug-cc-pVDZ has been found to be sufficient for structural predictions, much higher basis sets and highly correlated methods of the CCSD(T) type - that is, the higher angular momentum functions, diffuse functions and methodologies important for calculating dispersion contributions - were required for accurate prediction of binding energies ${ }^{244-246}$ suggesting that dispersion energy is the dominant energy contribution.
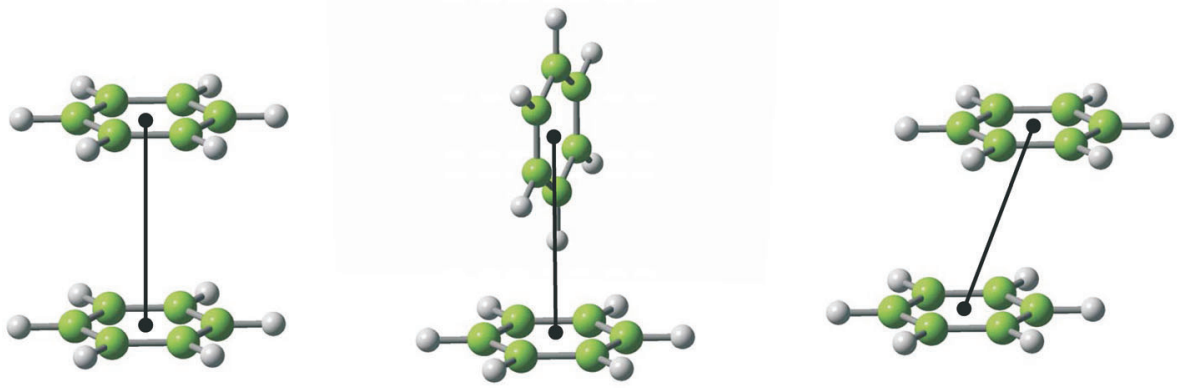

FIG. 20

Parallel (sandwich), T-shaped, and parallel displaced structures of benzene dimer 
As a further curiosity, it may be noted that the third isomer of the dimer, the parallel-displaced structure (see Fig. 20), is predicted by these later studies to be extremely close in energy to the T-shaped structure, and may in fact be the global minimum geometry. Thus, we must further consider the relative exchange-repulsion energy of the parallel-displaced and T-shaped isomers as well as the relative quadrupole-quadrupole and dispersion contributions as a delicate balance of energy contributions in deciding the true global minimum. Whilst it does seem clear from experiment that the T-shaped structure is the true global minimum, it is obvious that the benzene dimer, as a prototype of $\pi-\pi$ type interactions, provides a sensitive test of current computational models and will continue presenting a challenge to computational chemistry in the foreseeable future.

The quadrupole-quadrupole interaction discussed in the benzene dimer plays an important role not only in non-covalent complexes but is also important for the structure of proteins. The structure of crystalline phenylalanine is to a large extent determined by the interaction of benzene rings. In an investigation of the crystal structures of phenylalanine, a high occurrence of T-shaped and parallel-displaced structures of the benzene rings was found ${ }^{247}$. The quadrupole-quadrupole interaction is attractive only for these two structures; all other structures show repulsive quadrupole-quadrupole interaction.

\subsection{Nucleic Acid Base Pairs in vacuo}

The structure and dynamics of nucleic acid molecules are influenced by a variety of contributions. Among those, the interactions occurring between the nucleic acid base heterocycles are of particular importance. In DNA, the bases are involved in two qualitatively different mutual interaction types: $\mathrm{H}$-bonding and aromatic stacking. The $\mathrm{H}$-bonded base pair geometries observed in crystal structures of DNA fragments correspond to the minima on potential energy surfaces of isolated DNA base pairs. In contrast, stacked configurations present in crystals of DNA fragments are rather variable and in many cases do not correspond to energetically optimal stacked arrangements.

To study the intrinsic interactions of DNA bases experimentally, one needs to carry out accurate gas phase experiments. Gas phase experiments provide data giving insight into the physico-chemical origin of $\mathrm{H}$-bonding and stacking. However, experiments on DNA base pairs in vacuo are very difficult to perform. At this moment, we still have to rely on the field ionization mass spectroscopy data provided by Yanson, Teplitsky and 
Sukhodub ${ }^{84}$ for which there are problems regarding their interpretation and the comparison of these data with theoretical results. Let us further stress that there are still no other reliable gas phase experiments reporting on energetics of base pairing, even though any such experimental data would be of enormous value. The state-of-the-art gas-phase experiments $37 a, 186,248$ from the laboratories of de Vries and Kleinermanns give evidence only about the vibrational spectrum of a selected nucleic acid base pair but do not yield direct information on its structure or stabilization energy.

Experimental results on 9-methyladenine...1-methylthymine and 9-methylguanine...1-methylcytosine pairs were obtained from the temperature dependence of equilibrium constants measured at rather high temperatures (average temperatures were 323 and $381 \mathrm{~K}$ for $\mathrm{mA}$...mT and $\mathrm{mG}$...mC, respectively) and thus correspond to stabilization enthalpies at these temperatures ${ }^{84}$. After performing the first correlated ab initio calculations on the mA...mT and mG...mC complexes in the 1990's ${ }^{249,250}$, the resulting stabilization energies of the Hoogsteen and Watson-Crick structures, respectively, were compared with experimental data. The very good agreement obtained between theoretical and experimental data was claimed as evidence of the reliability of the theoretical procedure used. The question arises, however, whether this treatment was justified. Is it correct to compare the experimental value only with the (expected) most stable structure? The answer is no. The data ${ }^{84}$ show no evidence that only one structure was populated at the experimental temperatures. It must be expected that not only the most stable structures of the base pair but also many other structures should be indispensably populated. In the following paragraph we will show a correct procedure for comparison of theoretical data with the above mentioned experiment ${ }^{251}$.

Planar $\mathrm{H}$-bonded and stacked structures of the 9-methylguanine... 1-methylcytosine and 9-methyladenine...1-methylthymine were optimized at the RI-MP2 level using the TZVPP (5s3p2d1f/3s2p1d] basis set. The planar $\mathrm{H}$-bonded structure of the $\mathrm{mG}$...mC corresponds to the Watson-Crick (WC) arrangement, while the mA...mT possesses the Hoogsteen (H) structure. Stabilization energies for all structures were determined as the sum of the complete basis set limit of MP2 energies and the correlation $\left(\Delta \mathrm{E}^{\mathrm{CCSD}(\mathrm{T})}\right.$ $\left.\Delta \mathrm{E}^{\mathrm{MP2}}\right)$ correction term evaluated with the $6-31 \mathrm{G}^{* *}(0.25,0.15)$ basis set. The complete basis set limit was determined by a two-point extrapolation using the aug-cc-pVXZ basis sets for $X=D$ and $T$, and $T$ and $Q$, respectively. The convergence of the MP2 interaction energy for the studied complexes is rather slow and it is thus inevitable to include the extrapolation to the complete basis set limit. The M P2/aug-cc-pVQZ stabilization energies for all 
complexes were already very close to the complete basis set limit. Much cheaper D-T extrapolation provided a complete basis set limit which is very close (by less than $0.7 \mathrm{kcal} / \mathrm{mol}$ ) to the accurate T-Q term and can be recommended for evaluation of complete basis set limits of more extended complexes (e.g. larger motifs of DNA). The convergence of the $\left(\Delta \mathrm{E}^{\mathrm{CCSD}(\mathrm{T})}-\right.$ $\Delta E^{M P 2}$ ) term is known to be faster than that of the MP2 or CCSD(T) correlation energies themselves, and the $6-31 G^{* *}(0.25,0.15)$ basis set provides reasonable values for planar $\mathrm{H}$-bonded as well as stacked structures ${ }^{252}$. Inclusion of $\mathrm{CCSD}(\mathrm{T})$ correlation corrections is inevitable for obtaining reliable relative values between planar $\mathrm{H}$-bonding and stacking interactions; neglect of these corrections results in large errors of $2.5-3.4 \mathrm{kcal} / \mathrm{mol}$ (in relative energies).

Final stabilization energies (in $\mathrm{kcal} / \mathrm{mol}$ ) for base pairs studied were very large (mA...mT H 16.3, mA...mT stacked 13.1, mG...mC WC 28.5 and mG...mC stacked $18.0 \mathrm{kcal} / \mathrm{mol})$, much larger than values previously published. These stabilization energies should be first corrected for the zero-point energies and then the temperature-dependent enthalpy terms should be added. Finally, each structure should be weighted according to its population at experimental conditions. On the basis of our previous calculations ${ }^{148}$ using MD/Q simulations with the Cornell et al. empirical potential, stacked structures of $\mathrm{mG} . . . \mathrm{mC}$ and $\mathrm{mA}$...mT pairs are populated about 21 and $81 \%$ ( $T=300$ and $400 \mathrm{~K}$, respectively, close to experimental conditions). It is thus evident that experimental stabilization enthalpy from ref. ${ }^{84}$ should be compared with the weighted average of stabilization enthalpies of all non-negligibly populated structures rather than with the energy of the most stable (in terms of $\Delta \mathrm{H}_{\mathrm{T}}{ }^{0}$ ) but rarely present structure (mA...mT Hoogsteen: 4.0\%, mG...mC Watson-Crick: 28.2\%). Populations (weighting factors) were taken from MD/Q results ${ }^{148}$ and previous low-level ab initio interaction energies were scaled. $\mathrm{H}$-bonding and stacking energies were scaled ${ }^{251}$ by the ratio of the calculated $\Delta \mathrm{H}_{\mathrm{T}}{ }^{0}$ and the respective low-level value from ref. ${ }^{148}$, while the T-shaped structures were scaled by a factor taken as the average of the factors found for $\mathrm{H}$-bonding and stacking. The resulting interaction enthalpies, $\Delta \mathrm{H}^{0}{ }_{323 \mathrm{~K}}=-11.3 \mathrm{kcal} / \mathrm{mol}(\mathrm{mA} \ldots \mathrm{mT})$ and $\Delta \mathrm{H}^{0}{ }_{381 \mathrm{~K}}=-18.0 \mathrm{kcal} / \mathrm{mol}(\mathrm{mG} . . . \mathrm{mC})$, are in good agreement with experiment $\left(\Delta \mathrm{H}^{0}{ }_{323 \mathrm{~K}}=-13.0 \mathrm{kcal} / \mathrm{mol}(\mathrm{mA} \ldots \mathrm{mT})\right.$ and $\Delta \mathrm{H}^{0}{ }_{381 \mathrm{~K}}=-21.0 \mathrm{kcal} / \mathrm{mol}$ $(\mathrm{mG} . . \mathrm{mC}))$. Here we would like to point out that the present theoretical values are considerably larger than those published previously.

On the basis of error analysis we expect the present $\mathrm{H}$-bonding energies to be close to the true values, whilst stacked energies are still by about $10 \%$ smaller. The stacking energies for the mG...mC pair are considerably lower 
than the respective $\mathrm{H}$-bonding energies but they are larger than the $\mathrm{mA}$...mT $\mathrm{H}$-bonding energies. Present stabilization energies for the $\mathrm{H}$-bonding and stacking energies are the most accurate and reliable values and can be considered as new reference data. The conclusion about the importance of the stacking interaction can change our view on the importance of specific $\mathrm{H}$-bonding interactions and non-specific stacking interactions in nature. This concerns not only DNA, RNA and other DNA architectures (like hairpin) but also amino acids and proteins. The stacking of amino acids in proteins is evidently much more important than it has been previously believed and, indeed, can form one of the dominant stabilizing contributions.

\subsubsection{Ultrafast Hydrogen Atom Transfer in Clusters of Aromatic Molecules Including Base Pairs}

Presently, only very few groups worldwide have been able to show that nucleic acid base pairs can be produced in a supersonic jet and that hole burning can be used to help distinguish the concomitant conformers. So far no threshold ionization spectra have been attempted except one experiment by Kim and co-workers, who have recently reported the photoionization efficiency spectra for adenine via the $S_{1}$ state ${ }^{253}$. The photochemical stability of DNA and nucleic acid base pairs has been a subject of considerable discussion for some time; it poses very interesting questions as to exactly why nature has chosen to make some species undergo very rapid non-radiative processes upon electronic excitation to the $S_{1}$ state $e^{254-256}$. Lifetimes and decay channels reported in the literature are quite controversial 257,258 , due in part to the currently available methods of study and the concomitant amenability of available data to various equally valid but contradictory interpretations; all that is currently clear is that we are dealing with some very interesting and important dynamical processes.

This quandary can be related to the very recent and very surprising discovery by Jouvet and co-workers of hydrogen atom transfer (in contrast to proton transfer) in phenol...ammonia clusters ${ }^{254}$ and other systems ${ }^{255}$. This hydrogen detachment channel seems to be a general feature of a variety of other aromatic molecules, including nucleic acid base prototypes such as indoles. It can be contemplated that this discovery will certainly have an important impact on the understanding of the excited state dynamics of biologically important systems. Sobolewski and Domcke 255 (see Fig. 21) have shown that hydrogen detachment and hydrogen transfer is driven by repulsive $n \sigma^{*}$ states and that the cross-section for hydrogen atom detachment 
and transfer depends on the position of the conical intersections produced by the interaction of the $n \sigma^{*}$ surface with the $\pi \pi^{*}, \pi \sigma^{*}$ and $S_{0}$ potential energy surfaces. Time-resolved studies by Fujii and co-workers on phenol...ammonia clusters have monitored the hydrogen atom transfer on the picosecond time-scale ${ }^{259}$. Such dynamics will be further accessible experimentally by femtosecond and picosecond photoelectron spectroscopy and picosecond ZEKE spectroscopy.

Another very important dynamic process is charge-transfer (CT), which may be studied by time-resolved femtosecond photoelectron spectroscopy (fs-TRPES). This method, which has been pioneered by Stolow ${ }^{260}$, allows one to follow the charge-transfer dynamics from an electronically excited doorway state $\alpha$ to CT state $\beta$ (see Fig. 22). The probe laser is then used to project this dynamics onto the surface of the corresponding cation, as depicted in Fig. 22 for conformers $\alpha^{+}$and $\beta^{+}$. Especially for hydrogen atom and proton transfer dynamics, this projection will result in the observation of photoelectrons of different kinetic energy, associated with either conformer $\alpha^{+}$or $\beta^{+}$. The evolution of the wave packet on the excited state surface will result in a time evolution of the transition probabilities to ionic conformers $\alpha^{+}$and $\beta^{+}$.

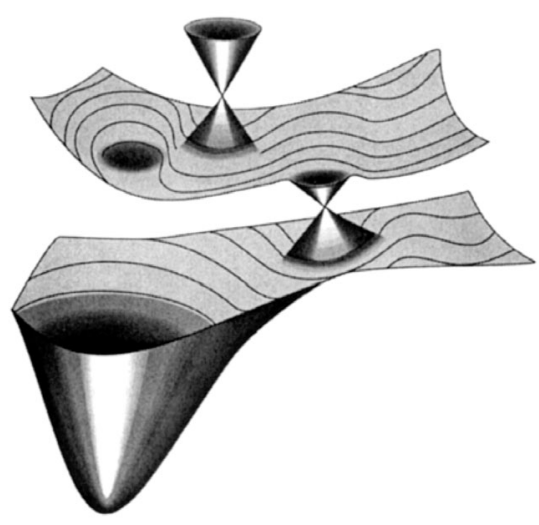

FIG. 21

Conical intersections produced by the interaction of the $n \sigma^{*}$ surface with the $\pi \pi^{*}, \pi \sigma^{*}$ and $\mathrm{S}_{0}$ potential energy surfaces. Reproduced from ref. ${ }^{255 a}$ with permission 
5.4. Biomolecules in the Gas Phase

\subsubsection{Photochemical Selectivity in Nucleic Acid Bases}

The photochemical stability of the genetic material and the components of DNA is quite surprising considering that, in comparison to a large variety of aromatic molecules, strong decay channels are present. A very topical question in this context is what is responsible for the photochemical stability: is it a property of the nucleic acid bases themselves, is it their attachment to sugar or phosphoric acid, or is it aided by the hydrogen bonds formed in the nucleic acid base pairs? The nucleic acid bases composing DNA exhibit rather short excited state lifetimes of the order of one picosecond or less. It has been argued that this rapid decay to the electronic ground state serves to protect these bases against photochemical damage because they do not cross into the reactive triplet state.

In a recent study256, 27 different DNA base pairs were studied by high-resolution UV spectroscopy and correlated ab initio calculations. Of these, 24 pairs exhibit sharp UV spectra and the remaining three pairs broad UV spectra. All the three structures were Watson-Crick ones and were characteristic by rapid internal conversion that makes these pairs uniquely stable. This finding suggests that, when constructing possible

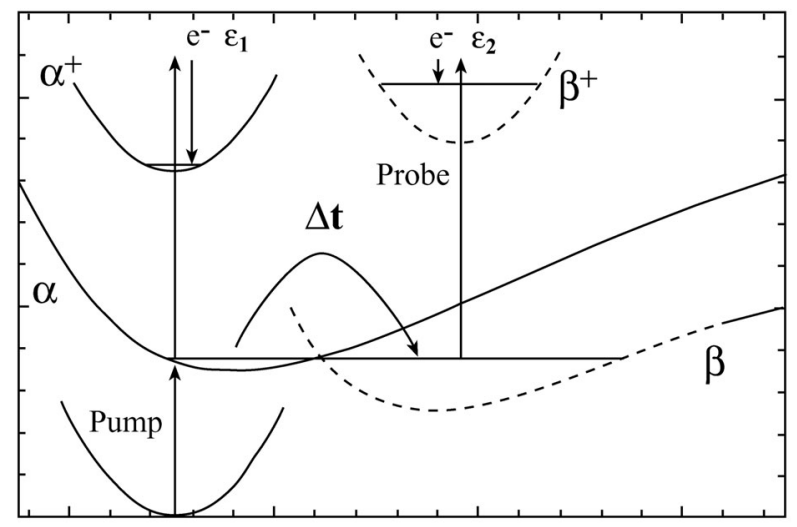

Reaction Coordinate

FIG. 22

Charge-transfer dynamics from an electronically excited doorway state $\alpha$ to CT state $\beta$. The probe laser is then used to project this dynamics onto the surface of the corresponding cation, as depicted for conformers $\alpha^{+}$and $\beta^{+}$. Reproduced from ref. ${ }^{260}$ with permission 
scenarios for prebiotic chemistry, the photostability of different DNA base pair structures should be considered. The special photostability of the Watson-Crick arrangements could have made an important contribution to the evolution of the recognition mechanism for the transfer of genetic code.

\subsection{Helium Nanodroplets: Formic Acid Dimer and Glycine Dimer}

As discussed earlier, the most stable structure of the formic acid dimer is without any doubt the cyclic structure with two strong $\mathrm{O}-\mathrm{H}$...O hydrogen bonds ${ }^{154}$. This structure exists dominantly in the gas phase and it was expected that it must also predominantly exist in He-droplet environment since this phase was believed to be close to a gas phase. The IR spectra of the dimer detected in He-droplets ${ }^{159}$ were, however, not consistent with the global minimum but supported the existence of a local minimum, also cyclic in structure but, instead, with one $\mathrm{O}-\mathrm{H} . . . \mathrm{O}$ and one $\mathrm{C}-\mathrm{H} . . . \mathrm{O}$ contact. We have shown that the dimer formed in He-droplet environment is controlled by electrostatic forces and the most stable structure corresponds to that with the most favourable orientation of dipoles ${ }^{159}$. These theoretical calculations revealed several reaction channels and the energetically most favourable one suggests a structure with one $\mathrm{O}-\mathrm{H} \ldots \mathrm{O}$ and one $\mathrm{C}-\mathrm{H} \ldots \mathrm{O}$ contact. Evidently, formation of a complex in a He-droplet is governed by factors other than those prevalent in molecular beams.

The situation for the glycine dimer, the IR spectra of which were also detected in He-droplets, is probably very similar. In Section 3.4. we discussed the disagreement between theoretical calculations and experimental findings ${ }^{156}$. We have shown that despite increasing the level of calculations, we were not able to interpret the experimental findings that showed the existence of a free $\mathrm{OH}$ group. We now believe the problem is not caused by inadequate theoretical treatment but it is due to the dimer, in He-droplet experiments, being formed in a completely different structure compared with that deduced from isolated molecule, gas-phase calculations. By analogy with the previous complex, the structure of the glycine dimer is believed to be determined by the long-range electrostatic interactions. These electrostatic forces are of long range and hence the two subsystems are already oriented preferentially according to their dipole (and quadrupole moments) when separated by very large distances. This preferential orientation leads to the formation of a dimer with a different energy minimum structure than that found in the gas phase; a similar mechanism of complex formation in the He-droplet environment has been previously described ${ }^{261}$. 


\section{MOLECULAR RECOGNITION AND SELF-ORGANIZATION}

\subsection{Recognition at the Molecular Level}

Understanding biological recognition on the molecular level is the conditio sine qua non for gaining a deeper knowledge of functionality in biological systems as well as of paths leading to new, more efficient drugs. The speed of development in this area is impressive but still there is a long way to go. The combination of experimental (calorimetry, X-ray structural analysis, NMR) and theoretical techniques (quantum chemistry and empirical potentials, Monte Carlo and molecular dynamics methods) is a powerful tool for investigating interactions between small and medium-size molecules and proteins and nucleic acids. For these interactions, it is highly desirable to dissect the Gibbs energy of the binding process into enthalpic and entropic parts. Moreover, the overall binding Gibbs energy is decomposed into several contributions, namely hydrophobic, non-covalent, and conformational. Both fundamental modes of non-covalent interaction between the drug and DNA have been considered: intercalation and minor groove binding. Only the former is associated with a significant conformational change. This work ${ }^{262}$ as well as the review ${ }^{263}$ contains a valuable list of references. The extensive review ${ }^{263}$ deals with several kinds of interactions between aromatic rings and an appropriate partner, which is essential for molecule-protein interactions. Specifically, interactions are considered between a proton donor (including weak proton donors) and a conjugated system, between cations of various sorts and a conjugated system and between different conjugated systems (stacking and other types of interaction). The semiempirical PM 3 method was used for calculations of enthalpy changes related to eight model systems for protein...ligand complexes ${ }^{264}$. Although good agreement was found between calculated and observed values, we are puzzled that this is the case when using a semiempirical procedure.

Although cation...conjugated systems are more common than analogue interactions with anions, anion recognition by $\pi$-acidic rings might be important, and not only in chemistry ${ }^{265}$. Interactions between (fluorinated) 1,3,5-triazine and small anions were studied at the MP2 level of theory. Besides the anion... $\pi$ contact, also hydrogen bonding interaction was considered.

A few more works on molecular recognition should be cited here: DNA recognition with alternative heterocycles (the benzimidazole-imidazole pair) ${ }^{266}$, sequence selectivity of molecular recognition between peptide $\beta$ 
sheets ${ }^{267}$, interactions between gases and biomolecules, which are simple models for studying molecular recognition 268 , and exploitation of NMR spectroscopy for the study of protein-ligand interactions ${ }^{269}$.

\subsection{Self-Assembly}

Several types of interactions are involved in self-assembly processes: various classes of hydrogen (and, e.g., halogen) bonding, hydrophobic forces, interactions between conjugated systems and metal ion templation. The preparation of drugs and drug carriers on the one hand and nanoscale devices for - perhaps - electronics on the other hand are distinguished reasons for an effort in this region.

For illustration, two different mechanisms are presented: the first one is the formation of linear supramolecular polymers from $\mathrm{N}, \mathrm{N}^{\prime}$-disubstituted ureas (with substituents which must not be too bulky) dissolved in heptane, which is due to the very strong, bifurcated hydrogen bonds between the urea moieties ${ }^{270}$. The second one is the self-association of sulfonated polymeric dialkynyldigold(I) with diphosphane ligands, which leads to macrocyclic ring complexes or to [2]catenanes ${ }^{270 b}$. This is in agreement with repeated observations that gold is valuable for building organometallic network polymers. The tendency to form Au...Au type bonds might be due to London forces (an analogy to the chalcogen-chalcogen interaction).

\section{OUTLOOK AND CONCLUSIONS}

One of the most exciting features of non-covalent interactions is that they are responsible for the structure and dynamics and, consequently, also the function of biological macromolecules such as DNA and proteins. Among various energy contributions, the London dispersion energy, for instance, in stacking interactions of aromatic rings, plays a much more important role than previously believed, and frequently it is even a dominant energy term. We have shown here, compared with our review in $2000^{6 a}$, that enormous advances have been achieved with the development of new theoretical and experimental methods for the study of non-covalent interactions. An ongoing and big challenge is the understanding of non-covalent interactions in biomolecules and their microsolvated clusters in the gas phase, including their ultrafast dynamics. More insight will develop from the more widespread application of new methods of laser evaporation of molecules of biological interest and their use in conjunction with a range of 
powerful laser-based experimental methods, both energy- as well as time-resolved, together with high-level computational techniques.

At the newly established Photon Science Institute at The University of Manchester, which provides a resourceful inter- and multidisciplinary environment, and at the Centre for Biomolecules and Complex Molecular Systems of the Academy of Sciences of the Czech Republic, we will continue developing and applying methods for the understanding of non-covalent interactions. The immense complexity of this field requires a more and more cross-disciplinary approach, inclusive of the life sciences and materials sciences, for instance. We are prepared for new and spectacular new insights in the next five years and we are looking forward to reviewing the field again then.

We gratefully acknowledge the contribution of our co-workers and students as cited in the references. Financial support provided from the Centre for Biomolecules and Complex Molecular Systems (project LC512 of Ministry of Education, Youth and Sports of the Czech Republic), and grants 203/05/0009 (Grant Agency of the Czech Republic) and A40055905 (Grant Agency of the Academy of Sciences of the Czech Republic) is acknowledged. K. Müller-Dethlefs acknowledges funding from the Engineering and Physical Sciences Research Council. The authors are grateful to Mark Watkins (Manchester) and Xin Tong (Manchester) for valuable discussions.

\section{REFERENCES}

1. van der Waals J. D.: Ph.D. Thesis. Leiden 1873. Quoted according to Brown L. M., Pais A., Pippard B. (Eds): Twentieth Century Physics, Vol. I. American Institute of Physics Press, New York 1995.

2. London F.: Z. Phys. Chem., B 1930, 11, 222.

3. Hellmann H.: Acta Physicochim. 1935, 2, 273.

4. Hirschfelder J. O., Curtiss C. F., Bird R. B.: Molecular Theory of Gases and Liquids. Wiley, New York 1954.

5. Hobza P., Zahradník R.: Intermolecular Complexes. Elsevier, Amsterdam 1988.

6. a) Müller-Dethlefs K., Hobza P.: Chem. Rev. 2000, 100, 143; b) Van der Waals Complexes I, II, III: Chem. Rev. 1988, 88; 1994, 94; 2000, 100.

7. Primas H.: Chemistry, Quantum Mechanics, and Reductionism, p. 344. Springer-Verlag, Berlin 1981.

8. a) Pauling L.: J. Am. Chem. Soc. 1931, 53, 1367; b) Pauling L.: The Nature of the Chemical Bond. Cornell University Press, Ithaca 1939.

9. a) Müller-Dethlefs K., Sander M., Schlag E. W.: Z. Naturforsch., A 1984, 39, 1089; b) Müller-Dethlefs K., Sander M., Schlag E. W.: Chem. Phys. Lett. 1984, 112, 291; c) Müller-Dethlefs K., Sander M., Chewter L. A. in: Laser Spectroscopy VII (T. W. Hänsch and Y. R. Shen, Eds), p. 118. Springer Verlag, Berlin 1985; d) Reiser G., Habenicht W., Müller-Dethlefs K., Schlag E. W.: Chem. Phys. Lett. 1988, 152, 119; e) Habenicht W., Reiser G., Müller-Dethlefs K.: J. Chem. Phys. 1991, 95, 4809; f) Müller-Dethlefs K.: J. Chem. Phys. 1991, 95, 4821. 
10. Dessent C. E. H., Haines S. R., Müller-Dethlefs K.: Chem. Phys. Lett. 1999, 315, 103.

11. Dessent C. E. H., Müller-Dethlefs K.: Chem. Rev. 2000, 100, 3999.

12. a) Kim W., Schaeffer M. W., Lee S., Chung J. S., Felker P. M.: J. Chem. Phys. 1999, 110, 11264; b) Lee S., Chung J. S., Felker P. M., Cacheiro J. L., Fernandez B., Pedersen T. B., Koch H.: J. Chem. Phys. 2003, 119, 12956; c) Lee S., Romascan J., Felker P. M., Pedersen T. B., Fernandez B., Koch H.: J. Chem. Phys. 2003, 118, 1230; d) Tanner C., Henseler D., Leutwyler S., Connell L. L., Felker P. M.: J. Chem. Phys. 2003, 118, 9157.

13. a) Garrett B. C., Dixon D. A., Camaioni D. M., Chipman D. M., Johnson M. A., Jonah C. D., Kimmel G. A., Miller J. H., Rescigno T. N., Rossky P. J., Xantheas S. S., Colson S. D., Laufer A. H., Ray D., Barbara P. F., Bartels D. M., Becker K. H., Bowen H., Bradforth S. E., Carmichael I., Coe J. V., Corrales L. R., Cowin J. P., Dupuis M., Eisenthal K. B., Franz J. A., Gutowski M. S., Jordan K. D., Kay B. D., LaVerne J. A., Lymar S. V., Madey T. E., McCurdy C. W., Meisel D., Mukamel S., Nilsson A. R., Orlando T. M., Petrik N. G., Pimblott S. M., Rustad J. R., Schenter G. K., Singer S. J., Tokmakoff A., Wang L. S., Wittig C., Zwier T. S.: Chem. Rev. 2005, 105, 355; b) Abou El-Nasr A., Fujii A., Ebata T., Mikami N.: Mol. Phys. 2005, 103, 1561; c) El-Hakam E. A., Ei-Nasr A., Fujii A., Yahagi T., Ebata T., Mikami N.: J. Phys. Chem. A 2005, 109, 2498; d) Imhof P., Krugler D., Brause R., Kleinermanns K.: J. Chem. Phys. 2004, 121, 2598; e) Wu R. H., Brutschy B.: J. Phys. Chem. A 2004, 108, 9715.

14. a) Jacoby C., Roth W., Schmitt M., Janzen C., Spangenberg D., Kleinermanns K.: J. Phys. Chem. A 1998, 102, 4471; b) Roth W., Schmitt M., Jacoby C., Spangenberg D., Janzen C., Kleinermanns K.: Chem. Phys. 1998, 239, 1; c) Schmitt M., Jacoby C., Gerhards M., Unterberg C., Roth W., Kleinermanns K.: J. Chem. Phys. 2000, 113, 2995; d) Schmitt M., Ratzer C., Meerts W. L.: J. Chem. Phys. 2004, 120, 2752; e) Westphal A., Jacoby C., Ratzer C., Reichelt A., Schmitt M.: Phys. Chem. Chem. Phys. 2003, 5, 4114.

15. a) Casaes R. N., Paul J. B., McLaughlin R. P., Saykally R. J., van Mourik T.: J. Phys. Chem. A 2004, 108, 10989; b) Huneycutt A. J., Casaes R. N., McCall B. J., Chung C. Y., Lee Y. P., Saykally R. J.: Chemphyschem 2004, 5, 321; c) Biennier L., Salama F., Allamandola L. J., Scherer J. J.: J. Chem. Phys. 2003, 118, 7863; d) Tan X. F., Salama F.: J. Chem. Phys. 2005, 122, 84318; e) Witkowicz E., Linnartz H., de Lange C. A., Ubachs W., Sfounis A., Massaouti M., Velegrakis M.: Int. J. Mass Spectrom. 2004, 232, 25.

16. a) Cole G. C., Legon A. C.: J. Chem. Phys. 2004, 121, 10467; b) Fowler P. W., Legon A. C., Thumwood J. M. A., Waclawik E. R.: Coord. Chem. Rev. 2000, 197, 231.

17. a) Giuliano B. M., Caminati W.: Angew. Chem., Int. Ed. 2005, 44, 603; b) Giuliano B. M., Ottaviani P., Caminati W., Schnell M., Banser D., Grabow J. U.: Chem. Phys. 2005, 312, 111; c) Alonso J. L., Antolinez S., Blanco S., Lesarri A., Lopez J. C., Caminati W.: J. Am. Chem. Soc. 2004, 126, 3244.

18. a) Caminati W., Lopez J. C., Alonso J. L., Grabow J. U.: Angew. Chem., Int. Ed. 2005, 44, 3840; b) Sanchez R., Blanco S., Lesarri A., Lopez J. C., Alonso J. L.: Chem. Phys. Lett. 2005, 401, 259; c) Lesarri A., Cocinero E. J., Lopez J. C., Alonso J. L.: Angew. Chem., Int. Ed. 2004, 43, 605; d) Blanco S., Lopez J. C., Lesarri A., Caminati W., Alonso J. L.: Mol. Phys. 2005, 103, 1473.

19. a) Goldman N., Leforestier C., Saykally R. J.: Philos. Trans. R. Soc. London, Ser. A 2005, 363, 493; b) Keutsch F. N., Cruzan J. D., Saykally R. J.: Chem. Rev. 2003, 103, 2533; c) Cruzan J. D., Braly L. B., Liu K., Brown M. G., Loeser J. G., Saykally R. J.: Science 1996, $271,59$. 
20. a) Chervenkov S., Wang P. Q., Braun J. E., Georgiev S., Neusser H. J., Nandi C. K., Chakraborty T.: J. Chem. Phys. 2005, 122, 244312; b) Georgiev S., Neusser H. J.: J. Electron Spectrosc. Relat. Phenom. 2005, 142, 207; c) Siglow K., Neusser H. J.: Chem. Phys. Lett. 2001, 343, 475.

21 a) Aguirre F., Pratt S. T.: J. Chem. Phys. 2004, 121, 9855; b) Hockridge M. R., Robertson E. G., Simons J. P., Borst D. R., Korter T. M., Pratt D. W.: Chem. Phys. Lett. 2001, 334, 31.

22. a) Berden G., Meerts W. L., Schmitt M., Kleinermanns K.: J. Chem. Phys. 1996, 104, 972;

b) Gerhards M., Schmitt M., Kleinermanns K., Stahl W.: J. Chem. Phys. 1996, 104, 967.

23. a) Kang C., Pratt D. W.: Int. Rev. Phys. Chem. 2005, 24, 1; b) Kang C. W., Yi J. T., Pratt D. W.: J. Chem. Phys. 2005, 123, 94306; c) Nguyen T. V., Korter T. M., Pratt D. W.: Mol. Phys. 2005, 103, 2453; d) Pratt D. W.: Science 2002, 296, 2347; e) Reese J. A., Nguyen T. V., Korter T. M., Pratt D. W.: J. Am. Chem. Soc. 2004, 126, 11387.

24. Lee Y. H., Jung J. W., Kim B., Butz P., Snoek L. C., Kroemer R. T., Simons J. P.: J. Phys. Chem. A 2004, 108, 69.

25. Borst D. R., Joireman P. W., Pratt D. W., Robertson E. G., Simons J. P.: J. Chem. Phys. 2002, 116, 7057.

26. a) Ford M. S., Tong X., Dessent C. E. H., Müller-Dethlefs K.: J. Chem. Phys. 2003, 119, 12914; b) Tong X., Ford M. S., Dessent C. E. H., Müller-Dethlefs K.: J. Chem. Phys. 2003, 119, 12908; c) Ford M. S., Müller-Dethlefs K.: Phys. Chem. Chem. Phys. 2004, 6, 23.

27. Riehn C., Lahmann C., Wassermann B., Brutschy B.: Chem. Phys. Lett. 1992, 197, 443.

28. a) Dian B. C., Clarkson J. R., Zwier T. S.: Science 2004, 303, 1169; b) Clarkson J. R., Dian B. C., Moriggi L., DeFusco A., McCarthy V., Jordan K. D., Zwier T. S.: J. Chem. Phys. 2005, 122, 214311.

29. a) Tanabe S., Ebata T., Fujii M., Mikami N.: Chem. Phys. Lett. 1993, 215, 347; b) Miyazaki M., Fujii A., Ebata T., Mikami N.: Science 2004, 304, 1134; c) Miyazaki M., Fujii A., Mikami N.: J. Phys. Chem. A 2004, 108, 8269; d) Venkatesan V., Fujii A., Mikami N.: Chem. Phys. Lett. 2005, 409, 57.

30. a) Gerhards M., Jansen A., Unterberg C., Kleinermanns K.: Chem. Phys. Lett. 2001, 344, 113; b) Hunig I., Seefeld K. A., Kleinermanns K.: Chem. Phys. Lett. 2003, 369, 173; c) Linder R., Nispel M., Haber T., Kleinermanns K.: Chem. Phys. Lett. 2005, 409, 260.

31. Honda M., Fujii A., Fujimaki E., Ebata T., Mikami N.: J. Phys. Chem. A 2003, 107, 3678. 32. a) Gerlach A., Unterberg C., Fricke H., Gerhards M.: Mol. Phys. 2005, 103, 1521; b) Gerhards M., Unterberg C., Gerlach A., Jansen A.: Phys. Chem. Chem. Phys. 2004, 6, 2682; c) Gerhards M.: Opt. Commun. 2004, 241, 493.

33. Gerhards M.: Private communication.

34. Doria A., Bartolini R., Feinstein J., Gallerano G. P., Pantell R. H.: IEEE J. Quantum Electron. 1993, 29, 1428.

35. Ding M. S., Weits H. H., Oepts D.: Nucl. Instrum. Methods Phys. Res., Sect. A 1997, 393, 504.

36. a) Carcabal P., Jockusch R. A., Hunig I., Snoek L. C., Kroemer R. T., Davis B. G., Gamblin D. P., Compagnon I., Oomens J., Simons J. P.: J. Am. Chem. Soc. 2005, 127, 11414; b) Jockusch R. A., Kroemer R. T., Talbot F. O., Snoek L. C., Carcabal P., Simons J. P., Havenith M., Bakker J. M., Compagnon I., Meijer G., von Helden G.: J. Am. Chem. Soc. 2004, 126, 5709.

37. a) Nir E., Plutzer C., Kleinermanns K., de Vries M.: Eur. Phys. J. D 2002, 20, 317; b) Seefeld K. A., Plutzer C., Lowenich D., Haber T., Linder R., Kleinermanns K., Tatchen J., 
Marian C. M.: Phys. Chem. Chem. Phys. 2005, 7, 3021; c) Brause R., Schmitt M., Krugler D., Kleinermanns K.: Mol. Phys. 2004, 102, 1615.

38. a) Abd El Rahim M., Antoine R., Arnaud L., Barbaire M., Broyer M., Clavier C., Compagnon I., Dugourd P., Maurelli J., Rayane D.: Rev. Sci. Instrum. 2004, 75, 5221; b) Chin W., Compagnon I., Dognon J. P., Canuel C., Piuzzi F., Dimicoli I., von Helden G., Meijer G., Mons M.: J. Am. Chem. Soc. 2005, 127, 1388; c) Polfer N. C., Paizs B., Snoek L. C., Compagnon I., Suhai S., Meijer G., von Helden G., Oomens J.: J. Am. Chem. Soc. $\mathbf{2 0 0 5}, 127,8571$.

39. Gregurick S. K., Chaban G. M., Gerber B.: Biophys. J. 2001, 80, 1256.

40. Chaban G. M., Jung J. O., Gerber R. B.: J. Chem. Phys. 1999, 111, 1823.

41. a) Yamada Y., Ebata T., Kayano M., Mikami N.: J. Chem. Phys. 2004, 120, 7400; b) Miyazaki M., Fujii A., Ebata T., Mikami N.: Chem. Phys. Lett. 2004, 399, 412; c) Yamada Y., Mikami N., Ebata T.: J. Chem. Phys. 2004, 121, 11530; d) Ebata T., Kayano M., Sato S., Mikami N.: J. Phys. Chem. A 2001, 105, 8623; e) Kayano M., Ebata T., Yamada Y., Mikami N.: J. Chem. Phys. 2004, 120, 7410.

42. a) Pitts J. D., Knee J. L.: J. Chem. Phys. 1998, 109, 7113; b) Pitts J. D., Knee J. L., Wategaonkar S.: J. Chem. Phys. 1999, 110, 3378; c) Zhang X., Knee J. L.: Discuss. Faraday Soc. 1994, 299.

43. a) Bellm S. M., Whiteside P. T., Reid K. L.: J. Phys. Chem. A 2003, 107, 7373; b) Davies J. A., Reid K. L., Towrie M., Matousek P.: J. Chem. Phys. 2002, 117, 9099; c) King A. K., Bellm S. M., Hammond C. J., Reid K. L., Towrie M., Matousek P.: Mol. Phys. 2005, 103, 1821.

44. a) Solca N., Dopfer O.: J. Phys. Chem. A 2001, 105, 5637; b) Solca N., Dopfer O.: J. Mol. Struct. 2001, 563-564, 241.

45. Ishiuchi S., Sakai M., Tsuchida Y., Takeda A., Kawashima Y., Fujii M., Dopfer O., Müller-Dethlefs K.: Angew. Chem., Int. Ed. 2005, 44, 6149.

46. a) Jurečka P., Hobza P.: Chem. Phys. Lett. 2002, 365, 89; b) Hobza P., Šponer J.: J. Am. Chem. Soc. 2002, 124, 11802; c) Jurečka P., Hobza P.: J. Am. Chem. Soc. 2003, 125, 15608.

47. Autumn K., Liang Y. A., Hsieh S. T., Zesch W., Chan W. P., Kenny T. W, Fearing R., Full R. J.: Nature 2000, 405, 681.

48. Boys S. F., Bernardi F.: Mol. Phys. 1970, 19, 553.

49. a) Buemi G., Gandolfo C.: J. Chem. Soc., Faraday Trans. 2 1989, 85, 215; b) Dannenberg J. J., Rios R.: J. Phys. Chem. 1994, 98, 6714.

50. a) Srinivasan R., Feenstra J. S., Park S. T., Xu S. J., Zewail A. H.: J. Am. Chem. Soc. 2004, 126, 2266; b) Xu S. J., Park S. T., Feenstra J. S., Srinivasan R., Zewail A. H.: J. Phys. Chem. A 2004, 108, 6650.

51. Caminati W.: Private communication.

52. Rowe W. F., Duerst R. W., Wilson E. B.: J. Am. Chem. Soc. 1976, 98, 4021.

53. Karlstrom G., Jonsson B., Roos B., Wennerstrom H.: J. Am. Chem. Soc. 1976, 98, 6851.

54. a) Gerhard D., Hellweg A., Merke I., Stahl W., Baudelet M., Petitprez D., Wlodarczak G.: J. Mol. Spectrosc. 2003, 220, 234; b) Schnell M., Grabow J. U., Hartwig H., Heineking N., Meyer M., Stahl W., Caminati W.: J. Mol. Spectrosc. 2005, 229, 1.

55. Ford M. S., Haines S. R., Pugliesi I., Dessent C. E. H., Müller-Dethlefs K.: J. Electron Spectrosc. Relat. Phenom. 2000, 112, 231.

56. Ford M., Lindner R., Müller-Dethlefs K.: Mol. Phys. 2003, 101, 705. 
57. a) Andrei H. S., Solca N., Dopfer O.: J. Phys. Chem. A 2005, 109, 3598; b) Lorenz U., Solca N., Dopfer O.: Chem. Phys. Lett. 2005, 406, 321; c) Solca N., Dopfer O.: Chem. Phys. Lett. 2003, 369, 68; d) Solca N., Dopfer O.: J. Am. Chem. Soc. 2004, 126, 9520.

58. Nelson D. D., Fraser G. T., Klemperer W.: J. Chem. Phys. 1985, 83, 6201.

59. Bunker P. R., Jensen P.: Molecular Symmetry and Spectroscopy, 2nd ed. NRC, Ottawa 1998.

60. Herzberg G.: Molecular Spectra and Molecular Structure, Vol. III, 2nd ed. Krieger Publishing Company, Malabar 1966.

61. Olthof E. H. T., van der Avoird A., Wormer P. E. S.: J. Mol. Struct. (THEOCHEM) 1994, 307, 201.

62. Cohen R. C., Saykally R. J.: Annu. Rev. Phys. Chem. 1991, 42, 369.

63. Huang Z. S., Miller R. E.: J. Chem. Phys. 1988, 88, 8008.

64. Wales D. J.: Energy Landscapes. Cambridge University Press, Cambridge 2003.

65. Fanourgakis G. S., Apra E., Xantheas S. S.: J. Chem. Phys. 2004, 121, 2655.

66. Fellers R. S., Braly L. B., Saykally R. J., Leforestier C.: J. Chem. Phys. 1999, 110, 6306.

67. Kim K., Jordan K. D.: J. Phys. Chem. 1994, 98, 10089.

68. Gregory J. K., Clary D. C.: Chem. Phys. Lett. 1994, 228, 547.

69. Gregory J. K., Clary D. C.: J. Chem. Phys. 1995, 102, 7817.

70. a) Burnham C. J., Xantheas S. S.: J. Chem. Phys. 2002, 116, 1500; b) Xantheas S. S., Burnham C. J., Harrison R. J.: J. Chem. Phys. 2002, 116, 1493.

71. Goldman N., Fellers R. S., Brown M. G., Braly L. B., Keoshian C. J., Leforestier C., Saykally R. J.: J. Chem. Phys. 2002, 116, 10148.

72. Car R., Parrinello M.: Phys. Rev. Lett. 1985, 55, 2471.

73. a) Felker P. M., Zewail A. H.: J. Chem. Phys. 1987, 86, 2460; b) Baskin J. S., Felker P. M., Zewail A. H.: J. Chem. Phys. 1987, 86, 2483.

74. a) Jarzeba W., Matylitsky V. V., Riehn C., Brutschy B.: Chem. Phys. Lett. 2003, 368, 680; b) Jarzeba W., Matylitsky V. V., Weichert A., Riehn C.: Phys. Chem. Chem. Phys. 2002, 4, 451.

75. Gauss J., Stanton J. F.: J. Chem. Phys. 2002, 116, 1773.

76. Dabkowska I., Jurečka P., Hobza P.: J. Chem. Phys. 2005, 122, 204322.

77. Jurečka P., Nachtigall P., Hobza P.: Phys. Chem. Chem. Phys. 2001, 3, 4578.

78. a) Feyereisen M., Fitzgerald G., Komornicki A.: Chem. Phys. Lett. 1993, 208, 359;

b) Vahtras O., Almlöf J., Feyereisen M.: Chem. Phys. Lett. 1993, 213, 514.

79. Bernholdt D. E., Harrison R. J.: Chem. Phys. Lett. 1996, 250, 477.

80. Ahlrichs R., Bär M., Häser M., Horn H., Kölmel C.: Chem. Phys. Lett. 1989, 162, 165.

81. Hobza P., Riehn Ch., Weichert A., Brutschy B.: Chem. Phys. 2002, 283, 331.

82. Simon S., Duran M., Dannenberg J. J.: J. Chem. Phys. 1996, 105, 11024.

83. Hobza P., Havlas Z.: Theor. Chem. Acc. 1998, 99, 372.

84. Yanson I. K., Teplitsky A. B., Sukhodub L. F.: Biopolymers 1979, 18, 1149.

85. Müller-Dethlefs K., Schlag E. W.: Annu. Rev. Phys. Chem. 1991, 42, 109.

86. Grant E. R., White M. G.: Nature 1991, 354, 249.

87. Merkt F., Softley T. P.: Int. Rev. Phys. Chem. 1993, 12, 205.

88. Müller-Dethlefs K., Dopfer O., Wright T. G.: Chem. Rev. 1994, 94, 1845.

89. Fischer I., Lindner R., Müller-Dethlefs K.: J. Chem. Soc., Faraday Trans. 1994, 90, 2425.

90. Müller-Dethlefs K. in: High Resolution Laser Photoionization and Photoelectron Studies (I. Powis, T. Baer and Ng C. Y., Eds), Chap. II, p. 22. John Wiley \& Sons Ltd. 1995

91. Müller-Dethlefs K., Schlag E. W., Grant E. R., Wang K., McKoy B. V.: Adv. Chem. Phys. 1995, 90, 1. 
92. Müller-Dethlefs K.: J. Electron Spectrosc. Relat. Phenom. 1995, 75, 35.

93. Müller-Dethlefs K., Cockett M. C. R. in: Nonlinear Spectroscopy for Molecular Structure Determination (R. W. Field, E. Hirota, J. P. Maier and S. Tsuchiya, Eds), pp. 164-199. IUPAC, Blackwell Science, Oxford 1998.

94. Müller-Dethlefs K., Schlag E. W.: Angew. Chem., Int. Ed. 1998, 37, 1346.

95. Cockett M. C. R., Müller-Dethlefs K., Wright T. G.: Annu. Rep. Prog. Chem., Sect. C 1998, $94,327$.

96. Merkt F.: Annu. Rev. Phys. Chem. 1997, 48, 675.

97. Wang K. S., McKoy V.: Annu. Rev. Phys. Chem. 1995, 46, 275.

98. a) Ullrich S., Tarczay G., Tong X., Dessent C. E. H., Müller-Dethlefs K.: Angew. Chem., Int. Ed. 2002, 41, 166; b) Ullrich S., Tarczay G., Tong X., Ford M. S., Dessent C. E. H., Müller-Dethlefs K.: Chem. Phys. Lett. 2002, 351, 121; c) Ullrich S., Tarczay G., Müller-Dethlefs K.: J. Phys. Chem. A 2002, 106, 1496; d) Ullrich S., Tong X., Tarczay G., Dessent C. E. H., Müller-Dethlefs K.: Phys. Chem. Chem. Phys. 2002, 4, 2897; e) Geppert W. D., Dessent C. E. H., Cockett M. C. R., Müller-Dethlefs K.: Chem. Phys. Lett. 1999, 303, 194; f) Geppert W. D., Dessent C. E. H., Ullrich S., Müller-Dethlefs K.: J. Phys. Chem. A 1999, 103, 7186.

99. Lindner R., Dietrich H.-J., Müller-Dethlefs K.: Chem. Phys. Lett. 1994, 228, 417.

100. Chupka W. A.: J. Chem. Phys. 1993, 98, 4520.

101. Zhu L. C., Johnson P.: J. Chem. Phys. 1991, 94, 5769.

102. Zhang X., Smith J. M., Knee J. L.: J. Chem. Phys. 1993, 99, 3133.

103. Dietrich H. J., Lindner R., Müller-Dethlefs K.: J. Chem. Phys. 1994, 101, 3399.

104. a) Chapman D. M., Müller-Dethlefs K., Peel J. B.: J. Chem. Phys. 1999, 111, 1955;

b) Haines S. R., Dessent C. E. H., Müller-Dethlefs K.: J. Chem. Phys. 1999, 111, 1947;

c) Haines S. R., Geppert W. D., Chapman D. M., Watkins M. J., Dessent C. E. H., Cockett M. C. R., Müller-Dethlefs K.: J. Chem. Phys. 1998, 109, 9244.

105. a) Braun J. E., Mehnert T., Neusser H. J.: Int. J. Mass Spectrom. 2000, 203, 1; b) Braun J. E., Neusser H. J., Harter P., Stockl M.: J. Phys. Chem. A 2000, 104, 2013; c) Chervenkov S., Wang P. Q., Braun J. E., Neusser H. J.: J. Chem. Phys. 2004, 121, 7169; d) Georgiev S., Chakraborty T., Neusser H. J.: J. Phys. Chem. A 2004, 108, 3304; e) Georgiev S., Neusser H. J.: Chem. Phys. Lett. 2004, 389, 24; f) Neusser H. J., Siglow K.: Chem. Rev. 2000, 100, 3921.

106. Dietrich H.-J., Müller-Dethlefs K., Baranov L. Y.: Phys. Rev. Lett. 1996, 76, 3530.

107. Pittner J., Hobza P.: Chem. Phys. Lett. 2004, 390, 496.

108. Dunning T. H.: J. Chem. Phys. 1989, 90, 1007.

109. Halkier A., Helgaker T., Jorgensen P., Klopper W., Koch H., Olsen J., Wilson A. K.: Chem. Phys. Lett. 1998, 286, 243.

110. Truhlar D. G.: Chem. Phys. Lett. 1998, 294, 45.

111. Lara-Castells M. P., Krems R. V., Buchachenko A. A., Delgado-Barrio G., Villarreal P.: J. Chem. Phys. 2001, 115, 10438.

112. Zahradník R., Šroubková L.: Isr. J. Chem. 2003, 43, 243.

113. a) van Mourik T., Dunning T. H., Jr.: J. Chem. Phys. 1997, 107, 2451; b) Schütz M., Brdarski S., Widmark P.-O., Lindh R., Karlström G.: J. Chem. Phys. 1997, 107, 4597; c) de Lara-Castells M. P., Krems R. V., Bucharenko A. A., Delgado-Barrio G., Villarreal P.: J. Chem. Phys. 2001, 115, 10438; d) Šroubková L., Zahradník R.: Helv. Chim. Acta 2001, 84, 1328; e) Zahradník R., Šroubková L.: Helv. Chim. Acta 2003, 86, 979; f) Zahradník R., Šroubková L.: Int. J. Quantum Chem. 2005, 104, 52. 
114. Braun J., Neusser H. J., Hobza P.: J. Phys. Chem. A 2003, 107, 3918.

115. a) Hobza P., Šponer J., Retschel T.: J. Comput.Chem. 1995, 16, 1315; b) Kristyan S., Pulay P.: Chem. Phys. Lett. 1994, 229, 175.

116. a) Liao M.-S., Lu Y., Parker V. D., Scheiner S.: J. Phys. Chem. A 2003, 107, 8939; b) Desplanches C., Ruiz E., Rodríguez-Fortea A., Alvarez S.: J. Am. Chem. Soc. 2002, 124, 5197; c) Zarić S. D.: Eur. J. Inorg. Chem. 2003, 2197; d) Elstner M., Hobza P., Frauenheim T., Suhai S., Kaxiras E.: J. Chem. Phys. 2001, 114, 5149; e) Cybulski S. M., Bledson T. M., Toczyłowski R. R.: J. Chem. Phys. 2002, 116, 11039; f) Bagno A., Saielli G.: Chem. Eur. J. 2003, 1486; g) Romano R. M., Downs A. J.: J. Phys. Chem. A 2003, 107, 5298.

117. a) Bartlett R. J., Grabowski I., Hirata S., Ivanov S.: The Exchange-Correlation Potential in $a b$ initio Density Functional Theory and Coupled-Cluster Theory, submitted; b) Grabowski I., Hirata S., Ivanov S., Bartlett R. J.: J. Chem. Phys. 2002, 116, 4415; c) Hirata S., Ivanov S., Grabowski I., Bartlett R. J.: J. Chem. Phys. 2002, 116, 6468; d) Misquitta A. J., Szalewicz K.: Chem. Phys. Lett. 2002, 357, 301; e) Misquitta A. J., Jeziorski B., Szalewicz K.: Phys. Rev. Lett. 2003, 91, 33201; f) Salazar M. C., Paz J. L., Hernández A. J., Manzanares C. I., Ludeňa E. V.: Int. J. Quantum Chem. 2003, 95, 177.

118. a) Vondrášek J., Bendová L., Klusák V., Hobza P.: J. Am. Chem. Soc. 2005, 127, 2615; b) Černý J., Hobza P.: Phys. Chem. Chem. Phys. 2005, 7, 1624.

119. Aliev M. R., Watson J. K. G. in: Molecular Spectroscopy: Modern Research, (K. Nahari Rao, Ed.), Vol. III. Academic Press, New York 1985.

120. Mills I. M. in: Molecular Spectroscopy: Modern Research, (K. Nahari Rao and C. W. Mathews, Eds), Vol. I., p. 115. Academic Press, New York 1972.

121. Maslen P. E., Handy N. C., Amos R. D., Jayatilaka D.: J. Chem. Phys. 1992, 97, 4233.

122. Handy N. C., Masle P. E., Amos R. D., Andrews J. S., Murray C. C. W., Laming G. J.: Chem. Phys. Lett. 1992, 197, 506.

123. Bludský O., Špirko V., Kobayashi R., Jorgensen P.: Chem Phys. Lett. 1994, 228, 568.

124. Špirko V., Šponer J., Hobza P.: J. Chem. Phys. 1997, 106, 1472.

125. Hobza P., Bludský O., Suhai S.: Phys. Chem. Chem. Phys. 1999, 1, 3073.

126. Elrod M. J., Saykally R. J.: J. Chem. Phys. 1995, 103, 933.

127. Miller R. E., Heijmen T. G. A., Wormer P. E. S., van der Avoird A., Moszynski R.: J. Chem. Phys. 1999, 110, 5651.

128. van der Sanden G. C. M., Wormer P. E. S., van der Aoird A., Schuttenmaer C. A., Saykally R. J.: Chem. Phys. Lett. 1994, 226, 22.

129. Cohen R. S., Saykally R. J.: J. Chem. Phys. 1991, 95, 7891.

130. Brauer B., Gerber R. B., Kabeláč M., Hobza P., Bakker J. M., Riziq A. G. A., de Vries M. S.: J. Phys. Chem. A 2005, 109, 6974.

131. a) Chang H.-C., Klemperer W.: J. Chem. Phys. 1993, 98, 9266; b) Chang H.-C., Klemperer W.: J. Chem. Phys. 1994, 100, 1; c) Klopper W., Quack M., Suhm M. A.: J. Chem. Phys. 1998, 108, 10096; d) Quack M., Suhm M. A.: Chem. Phys. Lett. 1990, 171, 517; e) Davis S., Anderson D. T., Nesbitt D. J.: J. Chem. Phys. 1996, 105, 6645; f) Salvador P., Szczęśniak M. M.: J. Chem. Phys. 2003, 118, 537; g) Farrell J. T. Jr., Sneh O., Nesbitt D. J.: J. Phys. Chem. 1994, 98, 6068; h) Tsang S. N., Chang H.-C., Klemperer W.: J. Phys. Chem. 1994, 98, 7313; i) Prosmiti R., Cunha C., Buchachenko A. A., Delgado-Barrio G., Villarreal P.: J. Chem. Phys. 2002, 117, 10019; j) Aquilanti V., Bartolomei M., Cappelletti D., Carmona-Novillo E., Pirani F.: J. Chem. Phys. 2002, 117, 615; k) Çarçabal P., Seurre N., Chevalier M., Broquier M., Brenner V.: J. Chem. Phys. 
2002, 117, 1522; l) Watkins M. J., Belcher D., Cockett M. C. R.: J. Chem. Phys. 2002, 116, 7868.

132. Marshall M. D., Pond B. V., Lester M. I.: J. Chem. Phys. 2003, 118, 1196.

133. a) Pivonka N. L., Kaposta C., von Helden G., Meijer G., Wöste L., Neumark D. M., Asmis K. R.: J. Chem. Phys. 2002, 117, 6493; b) Solca N., Dopfer O.: Chem. Eur. J. 2003, 9, 3154.

134. Aziz R. A.: J. Chem. Phys. 1993, 99, 4518.

135. Hutson J. M.: J. Chem. Phys. 1992, 96, 6752.

136. Hutson J. M.: J. Chem. Phys. 1988, 89, 4550.

137. Reimers J. R., Watts R. O., Klein M. L.: Chem. Phys. 1982, 64, 95.

138. Shoeib T., Ruggiero G. D., Siu K. W. M., Hopkinson A. C., Williams I. H.: J. Chem. Phys. 2002, 117, 2762.

139. Bakowies D.: Nachr. Chem. 2003, 51, 325.

140. Amar F. G., Berry R. S.: J. Chem. Phys. 1986, 85, 5943.

141. Cornell W. D., Cieplak P., Bayly C. I., Gould I. R., Merz K. M. Jr., Ferguson D. M., Spellmeyer D. C., Fox T., Caldwell J. W., Kollman P. A.: J. Am. Chem. Soc. 1995, 117, 5179.

142. a) Hobza P., Šponer J.: Chem. Rev. 1999, 99, 3247; b) Šponer J., Hobza P.: Collect. Czech. Chem. Commun. 2003, 68, 2231.

143. Kratochvíl M., Engkvist O., Šponer J., Jungwirth P., Hobza P.: J. Phys. Chem. 1998, 102, 6921.

144. Ryjáček F., Kratochvíl M., Hobza P.: Chem. Phys. Lett. 1999, 313, 393.

145. Kratochvíl M., Engkvist O., Jungwirth P., Hobza P.: Phys. Chem. Chem. Phys. 2000, 2, 2419.

146. Kratochvíl M., Šponer J., Hobza P.: J. Am. Chem. Soc. 2000, 122, 3495.

147. Ryjáček F., Engkvist O., Vacek J., Kratochvíl M., Hobza P.: J. Phys. Chem. A 2001, 105, 1197.

148. Kabeláč M., Hobza P.: J. Phys. Chem. B 2001, 105, 5804.

149. a) Kabeláč M., Hobza P.: Chem. Eur. J. 2001, 7, 2067; b) Kabeláč M., Zendlová L., Řeha D., Hobza P.: J. Phys. Chem. 2005, 109, 12206.

150. Trygubenko S. A., Bogdan T. V., Rueda M., Orozco M., Luque F. J., Šponer J., Slavíček P., Hobza P.: Phys. Chem. Chem. Phys. 2002, 4, 4192.

151. Hanus M., Ryjáček F., Kabeláč M., Kubar T., Bogdan T. V., Trygubenko S. A., Hobza P.: J. Am. Chem. Soc. 2003, 125, 7678.

152. Hanus M., Kabeláč M., Rejnek J., Ryjáček F., Hobza P.: J. Phys. Chem. B 2004, 108, 2087.

153. Rejnek J., Hanus M., Kabeláč M., Ryjáček F., Hobza P.: Phys. Chem. Chem. Phys. 2005, 7, 2006.

154. Chocholoušová J., Vacek. J., Hobza P.: Phys. Chem. Chem. Phys. 2002, 4, 2119.

155. Chocholoušová J., Vacek J., Hobza P.: J. Phys. Chem. A 2003, 107, 3086.

156. Chocholoušová J., Vacek J., Huisken F., Werhahn O., Hobza P.: J. Phys. Chem. A 2002, 106, 11540.

157. Gantenberg M., Halupka M., Sander W.: Chem. Eur. J. 2000, 6, 1865.

158. Brutschy B.: Personal communication, 2000.

159. Madeja F., Havenith M., Nauta K., Miller R. E., Chocholoušová J., Hobza P.: J. Chem. Phys. 2004, 120, 10554.

160. a) Luo F., McBane G. C., Kim G., Giese C. F., Gentry W. R.: J. Chem. Phys. 1993, 98, 3564; b) Liu B., McLean A. D.: J. Chem. Phys. 1989, 91, 2348; c) Vos R. J., van Lenthe 
J. H., van Duijneveldt F. B.: J. Chem. Phys. 1990, 93, 643; d) Aziz R. A., Slaman M. J.: J. Chem. Phys. 1991, 94, 8047; e) Slater J. C.: Phys. Rev. 1928, 32, 349; f) Chałasiński G., Gutowski M.: Chem. Rev. 1988, 88, 943; g) Klopper W., Noga J.: J. Chem. Phys. 1995, 103, 6127; h) Jaszuński M., Klopper W., Noga J.: J. Chem. Phys. 2000, 113, 71; i) Aziz R. A., Janzen A. R., Moldover M. R.: Phys. Rev. Lett. 1995, 74, 1586; j) Aziz R. A., Slaman M. J.: J. Chem. Phys. 1991, 94, 8047; k) Luo F., Giese C. F., Gentry W. R.: J. Chem. Phys. 1996, 104, 1151; 1) Toennies J. P. in: The Chem. Phys. of Atomic and Mol. Clusters (Proc. Int. School of Physic Enrico Fermi, Course 107) (G. Scoles, Ed.). Elsevier, Amsterdam 1990; m) Schöllkopf W., Toennies J. P.: J. Chem. Phys. 1996, 104, 1155; n) Aziz R. A., Meath W. J., Allnatt A. R.: Chem. Phys. 1983, 78, 295; o) Aziz R. A., Slaman M. J.: Chem. Phys. 1989, 130, 187; p) Aziz R. A., Slaman M. J.: Mol. Phys. 1986, 58, 679; q) Tang K. T., Toennies J. P.: J. Chem. Phys. 2003, 118, 4976; r) Laschuk E. F., Martins M. M., Evangelisti S.: Int. J. Quantum Chem. 2003, 95, 303; s) van Mourik T., Gdanitz R. J.: J. Chem. Phys. 2002, 116, 9620.

161. a) Wei J., Zhuo S., Ju G.: Int. J. Quantum Chem. 2004, 97, 977; b) Tao Ch., Dagdigian P. J.: J. Chem. Phys. 2003, 118, 1242; c) Kłos J., Chałasiński G., Krems R. V., Buchachenko A. A., Aquilanti V., Pirani F., Cappelletti D.: J. Chem. Phys. 2002, 116, 9269; d) Meyer W., Hariharan P. C., Kutzelnigg W.: J. Chem. Phys. 1980, 73, 1880; e) Prosmiti R., Cunha C., Villarreal P., Delgado-Barrio G.: J. Chem. Phys. 2002, 116, 9249; f) Nauta K., Miller R. E.: J. Chem. Phys. 2001, 115, 10138; g) Hutson J. M.: J. Phys. Chem. 1992, 96, 4237; h) Lotrich V. F., van der Avoird A.: J. Chem. Phys. 2003, 118, 1110; i) Buchachenko A. A., Szczęśniak M. M., Kłos J., Chałasiński G.: J. Chem. Phys. 2002, 117, 2629; j) de Clercq H. L., Hendricks J. H., Bowen K. H.: J. Chem. Phys. 2002, 117, 2619; k) Jakowski J., Chałasiński G., Cybulski S. M., Szczęśniak M. M.: J. Chem. Phys. 2003, 118, 2731; l) Geleijns M., van der Avoird A., Wormer P. E. S., Halberstadt N.: J. Chem. Phys. 2002, 117, 7562.

162. a) Chang H.-C., Klemperer W.: J. Chem. Phys. 1996, 104, 7830; b) Bende A., Knapp-Mohammady M., Suhai S.: Int. J. Quantum Chem. 2003, 92, 152; c) Mayer I.: Int. J. Quantum Chem. 1983, 23, 341; d) Prosmiti R., Cunha C., Villarreal P., Delgado-Barrio G.: J. Chem. Phys. 2002, 117, 7017; e) Zhang Q., Chenyang L., Ma Y., Fish F., Szczęśniak M. M., Buch V.: J. Chem. Phys. 1992, 96, 6039; f) McDowell S. A. C.: J. Chem. Phys. 2003, 118, 4066; g) Engdahl A., Karlström G., Nelander B.: J. Chem. Phys. 2003, 118, 7797; h) Moskowitz J. W., Bačić Z., Sarsa A., Schmidt K. E.: J. Chem. Phys. 2001, 114, 10294; i) Van Ginderen P., Herrebout W. A., van der Veken B. J.: J. Phys. Chem. A 2003, 107, 5391; j) Maseras F., Morokuma K.: J. Comput. Chem. 1995, 16, 1170; k) Hopkins B. W., Tschumper G. S.: Int. J. Quantum Chem. 2004, 96, 294; 1) Lesarri A., Cocinero E. J., López J. C., Alonso J. L.: Angew. Chem., Int. Ed. 2004, 43, 605; m) Qu J. Y., Li W., Guo R., Zhao X. S.: J. Chem. Phys. 2002, 117, 2592; n) Kisiel Z., Pietrewicz B. A., Pszczółkowski L.: J. Chem. Phys. 2002, 117, 8248; o) Nemukhin A. V., Grigorenko B. L., Khriachtchev L., Tanskanen H., Pettersson M., Räsänen M.: J. Am. Chem. Soc. 2002, 124, 10706; p) Timerghazin Q. K., Nguyen T.-N., Peslherbe G. H.: J. Chem. Phys. 2002, 116, 6867; r) Valdés H., Sordo J. A.: J. Phys. Chem. A 2003, 107, 7845; s) Kryachko E. S., Zeegers-Huyskens T.: J. Phys. Chem. A 2003, 107, 7546; t) Chen H.-Y., Sheu W.-S.: J. Chem. Phys. 2001, 115, 10678; u) Prosmiti R., Villarreal P., Delgado-Barrio G.: J. Phys. Chem. A 2003, 107, 4768.

163. Ichieda N., Kasuno M., Banu K., Kihara S., Nakamatsu H.: J. Phys. Chem. A 2003, 107, 7597. 
164. a) Janda K. C., Hemminger J. C., Winn J. S., Novick S. E., Harris S. J., Klemperer W.: J. Chem. Phys. 1975, 63, 1419; b) Steed J. M., Dixon T. A., Klemperer W.: J. Chem. Phys. 1979, 70, 4940; c) Arunan E., Gutowsky H. S.: J. Chem. Phys. 1993, 98, 4294; d) Hobza P., Selzle H. L., Schlag E. W.: J. Am. Chem. Soc. 1994, 116, 3500; e) Hobza P., Selzle H. L., Schlag E. W.: J. Phys. Chem. 1996, 100, 18790; f) Jaffe R. L., Smith G. D.: J. Chem. Phys. 1996, 105, 2780; g) Tsuzuki S., Lüthi H. P.: J. Chem. Phys. 2001, 114, 3949; h) Sinnokrot M. O., Valeev E. F., Sherrill C. D.: J. Am. Chem. Soc. 2002, 124, 10887; i) Lee N. K., Park S., Kim S. K.: J. Chem. Phys. 2002, 116, 7902; j) Lee N. K., Park S., Kim S. K.: J. Chem. Phys. 2002, 116, 7910; k) Heidenreich A., Even U., Jortner J.: J. Chem. Phys. 2001, 115, 10175; 1) Hübner O., Glöß A., Klopper W. in: Electron Correlation: $a b$ initio Methods and Density Functional Theory, Bad Herrenalb, Germany, July 16-19, 2003, p. 27; m) Arunan E., Emilsson T., Gutowsky H. S., Fraser G. T., de Oliveira G., Dykstra C. E.: J. Chem. Phys. 2002, 117, 9766; n) Schäfer M., Pratt D. W.: J. Chem. Phys. 2001, 115, 11147; o) Schäfer M., Kang Ch., Pratt D. W.: J. Phys. Chem. A 2003, 107, 10753; p) Reimann B., Buchhold K., Barth H.-D., Brutschy B., Tarakeshwar P., Kim K. S.: J. Chem. Phys. 2002, 117, 8805; q) Chelli R., Gervasio F. L., Procacci P., Schettino V.: J. Am. Chem. Soc. 2002, 124, 6133; r) Park J. M., Tarakeshwar P., Kim K. S., Clark T.: J. Chem. Phys. 2002, 116, 10684; s) Jalbout A. F., Adamowicz L.: J. Chem. Phys. 2002, 116, 9672; t) Le Barbu K., Schiedt J., Weinkauf R., Schlag E. W., Nilles J. M., Xu S.-J., Thomas O. C., Bowen K. H.: J. Chem. Phys. 2002, 116, 9663; u) Lenoir D.: Angew. Chem., Int. Ed. 2003, 42, 854.

165. Gálvez O., Gómez P. C., Pacios L. F.: J. Chem. Phys. 2001, 115, 11166.

166. Desiraju G. R., Steiner T.: The Weak Hydrogen Bond. Oxford University Press, Oxford 1999.

167. Scheiner S.: Hydrogen Bonding. Oxford University Press, New York 1997.

168. Jeffrey G. A.: An Introduction to Hydrogen Bonding. Oxford University Press, New York 1997.

169. Suzuki S., Green P. G., Bumgarner R. E., Dasgumpta S., Goddard III W. A., Blake G. A.: Science 1992, 257, 942.

170. Pribble R. N., Garret A. W., Haber K., Zwier T. S.: J. Chem. Phys. 1995, 103, 531.

171. Djafari S., Lembach G., Barth H.-D., Brutschy B.: Z. Phys. Chem. 1996, 195, 253.

172. Djafari S., Barth H.-D., Buchhold K., Brutschy B.: J. Chem. Phys. 1997, 107, 10573.

173. Djafari S., Barth H.-D., Buchhold K., Brutschy B.: J. Chem. Phys. 1997, 107, 10573.

174. Legon A. C., Roberts B. P., Wallwork A. L.: Chem. Phys. Lett. 1990, 173, 107.

175. Steiner T., Desiraju G. R.: Chem. Commun. 1998, 891.

176. a) Steiner T., Tamm M., Grzegorzewski A., Schulte N., Veldman N., Schreurs A. M. M., Kanters J. A., Kroon J., van der Maas J., Lutz B.: J. Chem. Soc., Perkin Trans. 2 1996, 2441; b) Steiner T., Saenger W: J. Am. Chem. Soc. 1992, 114, 7123; c) Steiner T.: J. Chem. Soc., Perkin 2 1995, 1321.

177. Trudeau G. T., Dumas J.-M., Dupuis P., Guerin M., Sandorfy C.: Top. Curr. Chem. 1980, 93, 91.

178. Buděšínský M., Fiedler P., Arnold Z.: Synthesis 1989, 858.

179. Boldeskul I. E., Tsymbal I. F., Ryltsev E. V., Latajka Z., Barnes A. J.: J. Mol. Struct. 1997, 167, 436.

180. a) Hobza P., Havlas Z.: Chem. Rev. 2000, 100, 4253; b) Hobza P., Havlas Z.: Theor. Chem. Acc. 2002, 108, 325.

181. Coulson C. A.: Res. Appl. Ind. 1957, 10, 149. 
182. Reed A. E., Curtiss L. A., Weinhold F.: Chem. Rev. 1988, 88, 899.

183. Hermansson K.: J. Phys. Chem. 2002, 106, 4695.

184. Hobza P.: Phys. Chem. Chem. Phys. 2001, 3, 2555.

185. Hobza P., Špirko V.: Phys. Chem. Chem. Phys. 2003, 5, 1290.

186. Nir E., Janzen Ch., Imhof P., Kleinermanns K., de Vries M. S.: Phys. Chem. Chem. Phys. 2002, 4, 740 .

187. van der Veken B. J., Herrebout W. A., Szostak R., Shepkin D. N., Havlas Z., Hobza P.: J. Am. Chem. Soc. 2001, 123, 12290.

188. Alabugin I. V., Manoharan M., Peabody S., Weinhold F.: J. Am. Chem. Soc. 2003, 125, 5973.

189. Hobza P., Špirko V., Selzle H. L., Schlag E. W.: J. Phys. Chem. A 1998, 102, 2501.

190. Hobza P., Špirko V., Havlas Z., Buchhold K., Reimann B., Barth H.-D., Brutschy B.: Chem. Phys. Lett. 1999, 299, 180.

191. Reimann B., Buchhold K., Vaupel S., Brutschy B., Havlas Z., Hobza P.: J. Phys. Chem. A 2001, 105, 5560 .

192. Hobza P., Havlas Z.: Chem. Phys. Lett. 1999, 303, 447.

193. Zierkiewicz W., Jurečka P., Hobza P.: ChemPhysChem 2005, 6, 609.

194. Scheiner S. in: Reviews in Computational Chemistry (K. B. Lipkowitz and D. B. Boyd, Eds), VCH Publishers, Inc., Weinheim 1991.

195. a) Steiner T.: Angew. Chem., Int. Ed. 2002, 41, 48; b) Morrison C. A., Siddick M. M.: Chem. Eur. J. 2003, 9, 628.

196. a) Langlet J., Caillet J., Berges J., Reinhardt P.: J. Chem. Phys. 2003, 118, 6157; b) Raugei S., Klein M. L.: J. Am. Chem. Soc. 2003, 125, 8992; c) Ranganathan A., Kulkarni G. U., Rao C. N. R.: J. Phys. Chem. A 2003, 107, 6073; d) van der Vaart A., Merz K. M., Jr.: J. Chem. Phys. 2002, 116, 7380.

197. a) Rudkevich D. M., Rebek J., Jr.: Eur. J. Org. Chem. 1999, 1991; b) Ashton P. R., Baldoni V., Balzani V., Credi A., Hoffmann H. D. A., Martínez-Díaz M.-V., Raymo F. M., Stoddart J. F., Venturi M.: Chem. Eur. J. 2001, 7, 3482; c) Nguyen V. T., Ahn P. D., Bishop R., Scudder M. L., Craig D. C.: Eur. J. Org. Chem. 2001, 4489; d) Berl V., Huc I., Khoury R. G., Lehn J.-M.: Chem. Eur. J. 2001, 7, 2810.

198. a) Curtiss L. A., Frurip D. J., Blander M.: J. Chem. Phys. 1979, 71, 2703; b) Köddermann T., Schulte F., Huelsekopf M., Ludwig R.: Angew. Chem., Int. Ed. 2003, 42, 4904; c) Mas E. M., Bukowski R., Szalewicz K.: J. Chem. Phys. 2003, 118, 4386; d) Mas E. M., Bukowski R., Szalewicz K.: J. Chem. Phys. 2003, 118, 4404; e) Ma B.-Q., Sun H.-L., Gao S.: Angew. Chem., Int. Ed. 2004, 43, 1374; f) Kuo J.-L., Ciobanu C. V., Ojamäe L., Shavitt I., Singer S. J.: J. Chem. Phys. 2003, 118, 3583; g) Omta A. W., Kropman M. F., Woutersen S., Bakker H. J.: Science 2003, 301, 347; h) Pal S., Sankaran N. B., Samanta A.: Angew. Chem., Int. Ed. 2003, 42, 1741; i) Larin A. V., Trubnikov D. N., Vercauteren D. P.: Int. J. Quantum Chem. 2003, 92, 71.

199. a) Howard B. J., Dyke T. R., Klemperer W.: J. Chem. Phys. 1984, 81, 5417; b) Dayton D. C., Jucks K. W., Miller R. E.: J. Chem. Phys. 1989, 90, 2631.

200. a) Fu A., Du D., Zhou Z.: Int. J. Quantum Chem. 2004, 97, 865; b) Cabaleiro-Lago E. M., Otero J. R.: J. Chem. Phys. 2002, 117, 1621; c) Pejov L.: Int. J. Quantum Chem. 2003, 92, 516; d) Dominiak P. M., Grech E., Barr G., Teat S., Mallinson P., Woźniak K.: Chem. Eur. J. 2003, 9, 963; e) Cole G. C., Legon A. C., Ottaviani P.: J. Chem. Phys. 2002, 117, 2790; f) Hunt S. W., Higgins K. J., Craddock M. B., Brauer C. S., Leopold K. R.: J. Am. Chem. Soc. 2003, 125, 13850; g) Wieczorek R., Dannenberg J. J.: J. Am. Chem. Soc. 2003, 
125, 14065; h) Pivonka N. L., Kaposta C., Brümmer M., Helden G. V., Meijer G., Wöste L., Neumark D. M., Asmis K. R.: J. Chem. Phys. 2003, 118, 5275; i) Westler W. M., Frey P. A., Lin J., Wemmer D. E., Morimoto H., Williams P. G., Markley J. L.: J. Am. Chem. Soc. 2002, 124, 4196; j) Boys S. F., Bernardi F.: Mol. Phys. 1970, 19, 372; k) Šroubková L., Zahradník R.: Helv. Chim. Acta 2001, 84, 1328.

201. a) Sutor D. J.: Nature 1962, 68; b) Wang B., Hinton J. F., Pulay P.: J. Phys. Chem. A 2003, 107, 4683; c) Oku K., Watanabe H., Kubota M., Fukuda S., Kurimoto M., Tsujisaka Y., Komori M., Inoue Y., Sakurai M.: J. Am. Chem. Soc. 2003, 125, 12739; d) Ramos Ch., Winter P. R., Stearns J. A., Zwier T. S.: J. Phys. Chem. A 2003, 107, 10280; e) George L., Sanchez-García E., Sander W.: J. Phys. Chem. A 2003, 107, 6850; f) Matsuura H., Yoshida H., Hieda M., Yamanaka S., Harada T., Shin-ya K., Ohno K.: J. Am. Chem. Soc. 2003, 125, 13910; g) Delanoye S. N., Herrebout W. A., van der Veken B. J.: J. Am. Chem. Soc. 2002, 124, 7490; h) Blatchford M. A., Raveendran P., Wallen S. L.: J. Phys. Chem. A 2003, 107, 10311; i) Parra R. D., Bulusu S., Zeng X. C.: J. Chem. Phys. 2003, 118, 3499; j) Noman A., Rahman M. M., Bishop R., Craig D. C., Scudder M. L.: Eur. J. Org. Chem. 2003, 72; k) Parsch J., Engels J. W.: J. Am. Chem. Soc. 2002, 124, 5664; l) Li X., Liu L., Schlegel H. B.: J. Am. Chem. Soc. 2002, 124, 9639; m) Karpfen A., Kryachko E. S.: J. Phys. Chem. A. 2003, 107, 9724; n) Donati A., Ristori S., Bonechi C., Panza L., Martini G., Rossi C.: J. Am. Chem. Soc. 2002, 124, 8778; o) Cannizzaro C. E., Houk K. N.: J. Am. Chem. Soc. 2002, 124, 7163; p) McDowell S. A. C.: J. Chem. Phys. 2003, 118, 7283; q) Zahradník R., Šroubková L.: Helv. Chim. Acta 2003, 86, 979.

202. a) Metrangolo P., Resnati G.: Chem. Eur. J. 2001, 7, 2511; b) Gleiter R., Werz D. B., Rausch B. J.: Chem. Eur. J. 2003, 9, 2676; c) Pedireddi V. R., Reddy D. S., Goud B. S., Craig D. C., Rae A. D., Desiraju G. R.: J. Chem. Soc., Perkin Trans. 2 1994, 2353; d) Sanz P., Yánez M., Mó O.: Chem. Eur. J. 2003, 9, 4548; e) Li Y.-L., Wang D., Phillips D. L.: J. Chem. Phys. 2002, 117, 7931; f) De Santis A., Forni A., Liantonio R., Metrangolo P., Pilati T., Resnati G.: Chem. Eur. J. 2003, 9, 3974.

203. a) Matta Ch. F., Hernández-Trujillo J., Tang T.-H., Bader R. F. W.: Chem. Eur. J. 2003, 9, 1940; b) Bombicz P., Czugler M., Tellgren R., Kálmán A.: Angew. Chem., Int. Ed. 2003, 42, 1957; c) Havlas Z., Bauwe E., Zahradník R.: Chem. Phys. Lett. 1985, 121, 330.

204. Lee J. C., Peris E., Rheingold A. L., Crabtree R. H.: J. Am. Chem. Soc. 1994, 116, 11014.

205. a) Richardson T. B., De Gala S., Crabtree R. H.: J. Am. Chem. Soc. 1995, 117, 12875;

b) Klooster W. T., Koetzle T. F., Siegbahn P. E. M., Richardson T. B., Crabtree R. H.: J. Am. Chem. Soc. 1999, 121, 6337.

206. Liu Q., Hoffmann R.: J. Am. Chem. Soc. 1995, 117, 10108.

207. Zierkiewicz W., Hobza P.: Phys. Chem. Chem. Phys. 2004, 6, 5288.

208. a) Moisan S., Dannenberg J. J.: J. Phys. Chem. B 2003, 107, 12842; b) Salvador P., Kobko N., Wieczorek R., Dannenberg J. J.: J. Am. Chem. Soc. 2004, 126, 14190;

c) Viswanathan R., Asensio A., Dannenberg J. J.: J. Phys. Chem. A 2004, 108, 9205.

209. Asensio A., Kobko N., Dannenberg J. J.: J. Phys. Chem. A 2003, 107, 6441.

210. Richardson N. A., Wesolowski S. S., Schaefer III H. F.: J. Am. Chem. Soc. 2002, 124, 10163.

211. Kryachko E. S., Sabin J. R.: Int. J. Quantum Chem. 2003, 91, 695.

212. Kumar A., Elstner M., Suhai S.: Int. J. Quantum Chem. 2003, 95, 44.

213. Kawahara S., Uchimaru T.: Eur. J. Org. Chem. 2003, 2577.

214. Minakawa N., Kojima N., Hikishima S., Sasaki T., Kiyosue A., Atsumi N., Ueno Y., Matsuda A.: J. Am. Chem. Soc. 2003, 125, 9970. 
215. Stepanian S. G., Jalbout A. F., Hall C. S., Adamowicz L.: J. Phys. Chem. A 2003, 107, 7911.

216. Langer H., Doltsinis N. L.: J. Chem. Phys. 2003, 118, 5400.

217. a) Ciani B., Jourdan M., Searle M. S.: J. Am. Chem. Soc. 2003, 125, 9038; b) Kohtani M., Jarrold M. F.: J. Am. Chem. Soc. 2002, 124, 11148.

218. Papoian G. A., Ulander J., Wolynes P. G.: J. Am. Chem. Soc. 2003, 125, 9170.

219. Song X., Zhao X.: J. Chem. Phys. 2004, 120, 2005.

220. Laskin J., Futrell J. H.: J. Phys. Chem. A 2003, 107, 5836.

221. Kitova E. N., Bundle D. R., Klassen J. S.: J. Am. Chem. Soc. 2002, 124, 9340.

222. Huang J., Schlick T.: J. Chem. Phys. 2002, 117, 8573.

223. Lyman J. L., Müller G., Houston P. L., Piltch M., Schmid W. E., Kompa K. L.: J. Chem. Phys. 1985, 82, 810.

224. Chewter L. A., Müller-Dethlefs K., Schlag E. W.: Chem. Phys. Lett. 1987, 135, 219.

225. Brupbacher T., Bauder A.: Chem. Phys. Lett. 1990, 173, 435.

226. Sampson R. K., Lawrance W. D.: Aust. J. Chem. 2003, 56, 275.

227. Boesl U.: J. Phys. Chem. 1991, 95, 2949.

228. Gonohe N., Suzuki N., Abe H., Mikami N., Ito M.: Chem. Phys. Lett. 1983, 94, 549.

229. Schmidt M., Piuzzi F., Mons M., Lecalve J., Dimicoli I.: Inst. Phys. Conf. Ser. 1991, 199.

230. Knochenmuss R., Ray D., Hess W. P.: J. Chem. Phys. 1994, 100, 44.

231. Tong X., Müller-Dethlefs K.: Unpublished results.

232. Haines S. R., Dessent C. E. H., Müller-Dethlefs K.: J. Electron Spectrosc. Reatl. Phenom. 2000, $108,1$.

233. Tong X.: Ph.D. Thesis. University of York, York 2005.

234. Tong X., Müller-Dethlefs K., Dopfer O., Ishiuchi S., Sakai M., Tsuchida Y., Takeda A., Kawashima Y., Fujii M.: Unpublished results.

235. Dennis G. R., Richie L. D.: J. Phys. Chem. 1991, 95, 656.

236. Krause H., Ernstberger B., Neusser H. J.: Chem. Phys. Lett. 1991, 184, 411.

237. Krause H., Ernstberger B., Neusser H. J.: Ber. Bunsen-Ges. Phys. Chem. 1992, 96, 1183.

238. Scherzer W., Krätzschmar O., Selzle H. L., Schlag E. W.: Z. Naturforsch., A: Phys. Sci. 1992, 47, 1248.

239. Arunan E., Gutowsky H. S.: J. Chem. Phys. 1993, 98, 4294.

240. Hobza P., Selzle H. L., Schlag E. W.: Chem. Rev. 1994, 94, 1767.

241. Špirko V., Engvist O., Soldán P., Selzle H. L., Schlag E. W., Hobza P.: J. Chem. Phys. 1999, 111, 572 .

242. Tsuzuki S., Luthi H. P.: J. Chem. Phys. 2001, 114, 3949.

243. Karlstrom G., Linse P., Wallquist A., Jonsson B.: J. Am. Chem. Soc. 1983, 105, 3777.

244. Sinnokrot M. O., Valeev E. F., Sherrill C. D.: J. Am. Chem. Soc. 2002, 124, 10887.

245. Hopkins B. W., Tschumper G. S.: J. Phys. Chem. A 2004, 108, 2941.

246. Sinnokrot M. O., Sherrill C. D.: J. Phys. Chem. A 2004, 108, 10200.

247. Hunter C. A., Singh J., Thornton J. M.: J. Mol. Biol. 1991, $218,837$.

248. a) Nir E., Kleinermanns K., de Vries M. S.: Nature 2000, 408, 949; b) Nir E., Janzen C., Imhof P., Kleinermanns K., de Vries M. S.: J. Chem. Phys. 2001, 115, 4604; c) Nir E., Janzen C., Imhof P., Kleinermanns K., de Vries M. S.: Phys. Chem. Chem. Phys. 2002, 4, 732; d) Plützer Ch., Hünig P., Kleinermanns K.: Phys. Chem. Chem. Phys. 2003, 5, 1158.

249. Gould I. R., Kollman P. A.: J. Am. Chem. Soc. 1994, 116, 2493.

250. Šponer J., Leszczynski J., Hobza P.: J. Phys. Chem. 1996, 100, 5590.

251. Jurečka P., Hobza P.: J. Am. Chem. Soc. 2003, 125, 15608. 
252. a) Jurečka P., Hobza P.: Chem. Phys. Lett. 2002, 365, 89; b) Hobza P., Šponer J.: J. Am. Chem. Soc. 2002, 124, 11802.

253. Kang H., Jung B., Kim S. K.: J. Chem. Phys. 2003, 118, 6717.

254. a) Pino G. A., Dedonder-Lardeux C., Gregoire G., Jouvet C., Martrenchard S., Solgadi D.: J. Chem. Phys. 1999, 111, 10747; b) Pino G., Gregoire G., Dedonder-Lardeux C., Jouvet C., Martrenchard S., Solgadi D.: Phys. Chem. Chem. Phys. 2000, 2, 893; c) Dedonder-Lardeux C., Gregoire G., Jouvet C., Martrenchard S., Solgadi D.: Chem. Rev. 2000, $100,4023$.

255. a) Sobolewski A. L., Domcke W., Dedonder-Lardeux C., Jouvet C.: Phys. Chem. Chem. Phys. 2002, 4,1093; b) Perun S., Sobolewski A. L., Domcke W.: J. Am. Chem. Soc. 2005, 127, 6257; c) Schultz T., Samoylova E., Radloff W., Hertel I. V., Sobolewski A. L., Domcke W.: Science 2004, 306, 1765; d) Sobolewski A. L., Domcke W.: Chem. Phys. 2003, 294, 73; e) Sobolewski A. L., Domcke W.: J. Chem. Phys. 2005, 122, 184320.

256. Abo-Riziq A., Grace T., Nir E., Kabeláč M., Hobza P., de Vries M. S.: Proc. Natl. Acad. Sci. U.S.A. 2005, 102, 20.

257. Kang H., Lee K. T., Jung B., Ko Y. J., Kim S. K.: J. Am. Chem. Soc. 2002, 124, 12958. 258. Piuzzi F., Mons M., Dimicoli I., Tardivel B., Zhao Q.: Chem. Phys. 2001, 270, 205.

259. a) Daigoku K., Ishiuchi S., Sakai M., Fujii M., Hashimoto K.: J. Chem. Phys. 2003, 119, 5149; b) Ishiuchi S., Daigoku K., Hashimoto K., Fujii M.: J. Chem. Phys. 2004, 120, 3215; c) Ishiuchi S., Daigoku K., Saeki M., Sakai M., Hashimoto K., Fujii M.: J. Chem. Phys. 2002, 117, 7077; d) Ishiuchi S., Daigoku K., Saeki M., Sakai M., Hashimoto K., Fujii M.: J. Chem. Phys. 2002, 117, 7083.

260. Stolow A.: Annu. Rev. Phys. Chem. 2003, 54, 89.

261. Nauta K., Miller R. E.: Science 2000, 287, 293.

262. Haq I.: Arch. Biochem. Biophys. 2002, 403, 1.

263. Meyer E. A., Castellano R. K., Diederich F.: Angew. Chem., Int. Ed. 2003, 42, 1210.

264. Nikitina E., Sulimov V., Zayets V., Zaitseva N.: Int. J. Quantum Chem. 2004, 97, 747.

265. Mascal M., Armstrong A., Bartberger M. D.: J. Am. Chem. Soc. 2002, 124, 6274.

266. Briehn Ch. A., Weyermann P., Dervan P. B.: Chem. Eur. J. 2003, 9, 2110.

267. Nowick J. S., Chung D. M.: Angew. Chem., Int. Ed. 2003, 42, 1765.

268. Rudkevich D. M.: Angew. Chem., Int. Ed. 2004, 43, 558.

269. Homans S. W.: Angew. Chem., Int. Ed. 2004, 43, 290.

270. a) Lortie F., Boileau S., Bouteiller L.: Chem. Eur. J. 2003, 9, 3008; b) Mohr F., Jennings

M. C., Puddephatt R.: Eur. J. Inorg. Chem. 2003, 217. 